Physics Vol. 4, No. 1, pp. 1 - 64, 1968. Physics Publishing Co. Printed in Great Britain

\title{
PRINCIPLES AND METHODS OF DILUTION REFRIGERATION*
}

\author{
John C. WHEATLEY, O.E. VILCHES and W.R. ABEL \\ Department of Physics, University of California, \\ San Diego, La Jolla, California 92037, U.S.A.
}

\begin{abstract}
Following a brief introduction in Section $I$, the properties of pure He ${ }^{3}$ and of dilute solutions of $\mathrm{He}^{3}$ in superfluid $\mathrm{He}^{4}$ which are relevant to the design and understanding of dilution refrigerators are discussed in Section II. In Section III the basic model for discussing the thermodynamics of the dilution process is developed and applied to both a continuously operating refrigerator and to singlecycle refrigerators using both $\mathrm{He}^{3}$ flow and superfluid $\mathrm{He}^{4}$ flow. The relative merits of $\mathrm{He}^{3}$ flow and superleak operated devices are also mentioned. In Section IV the thermodynamical properties of $\mathrm{He}^{3}$ in the dilute phase of the refrigerator are calculated, including the dependence of concentration on temperature and the effective enthalpy function. The heat exchange problem is discussed in Section $V$, first by introducing new measurements of the Kapitza resistance for saturated dilute solutions and then by relating these to the concept of a $\mathrm{He}^{3}$-phonon resistance in the dilute solutions. Finally specific design estimates are made for the heat exchangers. In Section VI the physics of the $\mathrm{He}^{3}$ circulation problem is discussed. The effects of circulated $\mathrm{He}^{4}$ and of viscous heating due to $\mathrm{He}^{3}$ flow are also emphasized. Both the effects of thermal conduction and viscosity limit the low temperature attainable in a $\mathrm{He}^{3}$ flow type of dilution refrigerator. There is a lower limit to 'the temperature attainable as a result of these intrinsic factors which is calculated in Section VII.

The general characteristucs of the refrigeration device itself are reviewed in Section VIII. Section IX contains a detailed description of two systems built and tested by us. Parts of the apparatus discussed are the still, the heat exchangers, the mixing chamber, the condenser and main impedance, interconnections, and mechanical support. The auxiliary cryogenic and pumping systems are also described. In Section $X$ the operational characteristics of two dilution refrigerators are discussed, both in regard to starting them up and to their steady state operating characteristics. The effect of a heat load, of still power, and of the number of exchangers is given. Comparison with the thermodynamical treatment is made. Under suitable conditions a temperature of $10 \mathrm{~m}^{\circ} \mathrm{K}$ can be maintained continuously and a temperature of $4.5 \mathrm{~m}^{\circ} \mathrm{K}$ for short periods of time.
\end{abstract}

* This work has been supported by the U.S. Atomic Energy Commission, under contract AT(11-1)34, P.A. 143. 


\section{Introduction}

Iiv $1951 \mathrm{H}$. London [1] suggested a method for using solutions of $\mathrm{He}^{3}$ in superfluid $\mathrm{He}^{4}$ to produce refrigeration at low temperatures. In the proposed inethod the temperature would be reduced by allowing the $\mathrm{He}^{3}$ system to do work adiabatically at the expense of its kinetic energy. The proposal is based on a conception of the properties of $\mathrm{He}^{3}$ dissolved in superfluid He $\mathrm{H}^{4}$ due to Landau and Pomeranchuk $[2,3]$. The He ${ }^{3}$ particles are regarded as impurities with an energy spectrum which might be as simple as the ideal gas-like

$$
E=-E_{0_{3}}+p^{2} / 2 m^{*}+\ldots
$$

where $E_{0_{3}}$ is a binding energy, $p$ is $\mathrm{He}^{3}$ quasiparticle momentum, and $m^{*}$ is an effective mass. Following the discovery by Walters and Fairbank [4] of the phase separation phenomenon in $\mathrm{He}^{3}-$ $\mathrm{He}^{4}$ mixtures, analogous to the "condensation" of the Landau - Pomeranchuk $\mathrm{He}^{3}$ "gas", London, Clarke and Mendoza [5] proposed a more powerful method for producing refrigeration using $\mathrm{He}^{3}$ $\mathrm{He}^{4}$ solutions. After phase separation a $\mathrm{ie}^{3} \mathrm{rich}$ solution floats on top of $\mathrm{He}^{4}$ rich superfluid. The refrigeration is produced by "evaporation" of $\mathrm{He}^{3}$ from the upper phase into the superfluid lower phase. An important feature of this proposal is that the $\mathrm{He}^{3}$ may be continuously recirculated, refrigeration thereby being produced continuously.

The first partially successful device to produce cooling by dilution of $\mathrm{He}^{3}$ with $\mathrm{He}^{4}$ was reported by Das, de Bruyn Ouboter, and Taconis [ 6 ] who obtained a temperature of $0.22^{\circ} \mathrm{K}$. This temperature can also be reached by a carefully designed $\mathrm{He}^{3}$ refrigerator. The first really successful dilution refrigerator was built by liall, Ford and Thompson [7]. Their refrigerator, based on the "evaporation" idea and operating continuously, reached a temperature of $65 \mathrm{~m}^{\circ} \mathrm{K}$ $\left(1 \mathrm{~m}^{\circ} \mathrm{K} \equiv 10^{-3}{ }^{\circ} \mathrm{K}\right)$. Working independently, Neganov, Borisov and Liburg [3] built a continuously operating, high refrigeration capacity device which reached a temperature of $25 \mathrm{~m}{ }^{\circ} \mathrm{K}$. Vilches and Wheatley [9] built a device which reached a temperature of $20 \mathrm{~m}^{\circ} \mathrm{K}$ continuously and $14 \mathrm{~m}^{\circ} \mathrm{K}$ for short periods of time. In a second apparatus, operating noncontinuously and precooled by the continuously operating refrigerator, a temperature of $4.5 \mathrm{~m}^{\circ} \mathrm{K}$ was achieved [10].

A review of the principles of $\mathrm{He}^{3}-\mathrm{He}^{4}$ solutions and their application to dilution refrigeration has been given recently by one of us [11]. The reader is referred to this paper also for a discussion of the thermodynamics of processes occurring in the dilution refrigerator. In the present paper we present both general design principles and experiments relevant to the construction of dilution refrigerators. Our early work [9] was of great importance to us in recognizing a variety of important factors relating to refrigerator design. It will be discussed wherever appropriate, even though our latest refrigerator is capable of producing continuously a temperature of about $10 \mathrm{~m}^{\circ} \mathrm{K}$, twice as low as was obtained with our early version.

\section{Basic $\mathrm{He}^{3}-\mathrm{He}^{4}$ Properties}

The specific heat of a dilute solution of $\mathrm{He}^{3}$ in $\mathrm{He}^{4}$ is given within experimental error [12] by that of an ideal Fermi - Dirac gas $[13,14]$ provided the effective mass is allowed to increase weakly with $\mathrm{He}^{3}$ concentration. Some low temperature measurements are shown in Fig. 1. Bardeen, Baym and Pines (BBP) [15] estimate that in the limit of zero concentration the ratio of the effective mass of a $\mathrm{He}^{3}$ quasiparticle to the $\mathrm{He}^{3}$ atomic mass is $\mathrm{m}^{*} / \mathrm{m}_{3}=2.34$. This ratio being greater than unity is one of the ways in which the $\mathrm{He}^{4}$ background fluid manifests itself. For $\mathrm{He}^{3}$ concentrations of 5 or $6 \%$ the effective mass ratio has increased [12] to about 2.5 as a result of $\mathrm{He}^{3}-\mathrm{He}^{3}$ interactions [15]. The temperature dependence of the internal energy or 
the entropy of a dilute solution of fixed concentration may therefore be calculated with an accuracy of a few per cent using the work of Stoner [13] and McDougall and Stoner [14]. The input data are the effective mass ratio and the $\mathrm{He}^{3}$ number density. The latter may be obtained from the $\mathrm{He}^{3}$ concentration and the molar volume data of Kerr [16] and of Boghosian and Meyer $[17]$.

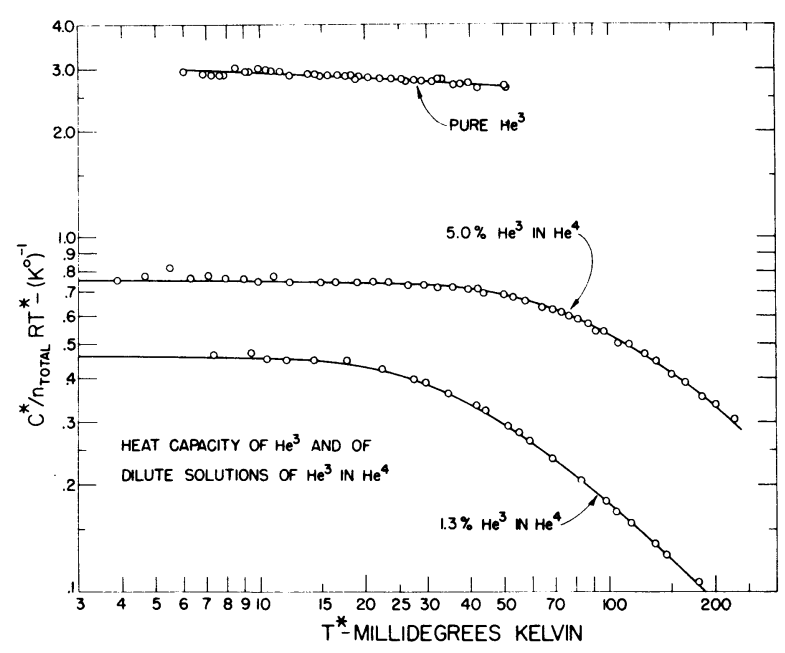

FIGURE 1

Ratio of heat capacity per mole of solution to $R T^{*}$, where $R$ is the gas constant and $T^{*}$ is the magnetic temperature, for dilute solutions of $\mathrm{He}^{3}$ in $\mathrm{He}^{4}$ of nominal concentration $1.3 \%$ and $5.0 \% \mathrm{He}^{3}$ and at saturated vapor pressure. Lines through the experimental data are theoretical ones based on the heat capacity of an ideal gas of Fermi - Dirac particles as computed in Refs. 13 and 14. For the nominal 1.3\% concentration the theoretical line was drawn for the parameters $x=0.0132, \mathrm{~m}^{*} / \mathrm{m}_{3}=2.38, T_{F}=0.141^{\circ} \mathrm{K}$. For the nominal $5 \%$ concentration these parameters are $x=0.0502, m^{*} / m_{3}=2.45, T_{F}=0.331^{\circ} \mathrm{K}$. The molar volumes determined in Ref. 16 were used in analyzing the data. Data of Ref. 38 for the heat capacity of pure $\mathrm{He}^{3}$ at 0.28 atm are shown for comparison.

For most applications of the dilution refrigerator it is sufficient to know the properties only of pure $\mathrm{He}^{3}$ and of dilute solutions of $\mathrm{He}^{3}$ in $\mathrm{He}^{4}$ for concentrations 1 ess than $10 \%$. This follows from examination of the phase separation diagram, Fig. 2, which was compiled from the work of various authors $[10,18-24]$. This diagram has the following significance. Imagine some mixture at a temperature of $1^{\circ} \mathrm{K}$. If $x$ is to the left of the $\lambda$ line the mixture is superfluid, if to the right, normal. For the sake of definiteness, imagine the initial mixture to be superfluid. Now let the temperature be lowered at constant $x$ until the left branch of the phase separation curve is reached. At this temperature $\mathrm{He}^{3}$ rich normal fluid starts to separate out above the rest of the solution. The concentration of the upper phase may be read off the right branch of the phase separation curve. On further reduction of the temperature the upper phase becomes richer in $\mathrm{He}^{3}$ while the lower phase becomes richer in $\mathrm{He}^{4}$. The $\mathrm{He}^{3}$ concentration in the 
two phases at any temperature is given by the intersection of a horizontal line representing the temperature in question with the right and left branches of the phase separation curve. The operating temperature of a dilution refrigerator is frequently below $0.2^{\circ} \mathrm{K}$. Examination of Fig. 2 shows that for this temperature and below the concentration of the lower phase is not in excess of $10 \% \mathrm{He}^{3}$ while the upper phase is essentially pure $\mathrm{He}^{3}$.

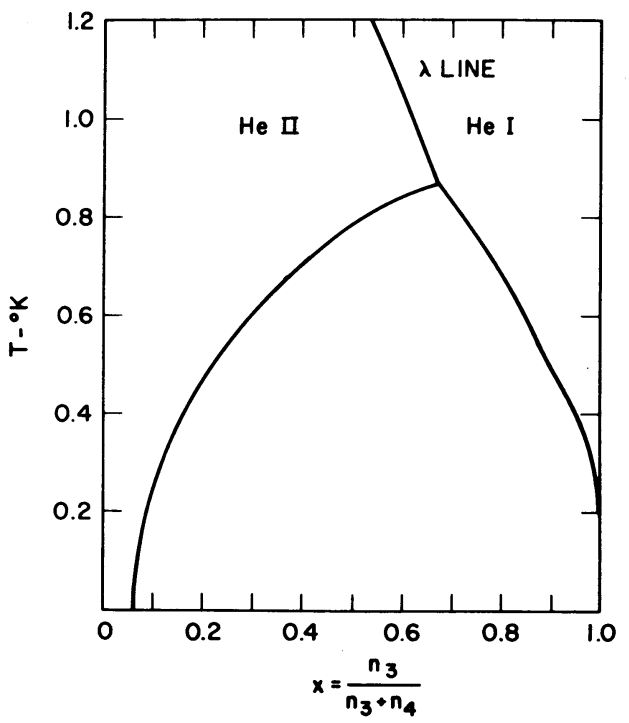

FIGURE 2

Phase separation diagram for $\mathrm{He}^{3}-\mathrm{He}^{4}$ mixtures at saturated vapor pressure as compiled using data from Refs. 10,18-24. The intersection with the $\lambda$ line is shown dividing mixtures into superfluid (He II) and normal fluid (He I).

It is a remarkable property of the equilibrium between $\mathrm{He}^{3}$ and dilute solutions, shown on Fig. 2, that as the temperature approaches zero the concentration of $\mathrm{He}^{3}$ in the dilute phase does not approach zero but rather approaches a constant value of about $6.3 \%[10,22]$. This property is of the greatest importance to the operation at low temperatures of the dilution refrigerator. It is responsible for the dilution refrigerator being a very powerful new tool rather than a scientific curiosity. A simple discussion of the finite solubility of $\mathrm{He}^{3}$ in $\mathrm{He}^{4}$ has been given in Ref. 11. However, the basic fact behind the finite solubility is that a single $\mathrm{He}^{3}$ quasiparticle is more strongly bound to liquid He${ }^{4}$ than it is to liquid $\mathrm{He}^{3}$.

Other properties of dilute solutions which are important to an understanding of the dilution refrigerator are $\mu_{3}$ and $\mu_{4}$, the partial chemical potentials per atom in the dilute phase for, respectively, $\mathrm{He}^{3}$ and $\mathrm{He}^{4}$. These two quantities are related by the thermodynamical relation 


$$
x\left(\frac{\partial \mu_{3} d}{\partial x}\right)_{T, P}+(1-x)\left(\frac{\partial \mu_{4}}{\partial x}\right)_{T, P}=0
$$

where $x$ is the $\mathrm{He}^{3}$ concentration. The quantity $\mu_{3_{d}}$ determines the left branch of the phase separation curve by the equation

$$
\mu_{3 d}(T, x)=\mu_{3}(T) ;(P \cong 0) \text {, }
$$

which expresses the thermodynamical requirement that the chemical potential per atom in the concentrated phase, $\mu_{3}$, be the same as that in the dilute phase. The quantity $\mu_{4}$ is important for dilution refrigerators since throughout the dilute phase, under steady state conditions when the superfluid is not accelerating, one has

$$
\vec{\nabla}_{4_{d}}(T, x)=0 \quad \text { (steady state) }
$$

This equation determines the concentration as a function of temperature once its concentration at some temperature is known, as it will be at the phase equilibrium temperature by equation (3). Equation (4) also is related to the setting up of pressure differences which are needed to drive $\mathrm{He}^{3}$ through the dilute phase.

It is desirable for purposes of design and analysis to have at least an approximate numerical equation for $\mu_{3}(T, x)$ and hence, using in part equation (2), for $\mu_{4}(T, x)$. Within the accuracy of knowledge of the properties of dilute solutions $\mu_{3_{d}}$ may be written

$$
\mu_{3_{d}}(T, x)=\mu_{3_{d}}(0, x)+\Delta \mu_{3_{d}}(T, x) .
$$

The quantity $\Delta \mu_{3}(T, x) \equiv \mu_{3}(T, x)-\mu_{3_{d^{\prime}}}(0, x)$ is the change in $\mu_{3}$ at temperature $T$ from its value at $T=0$ provided $x$ is rixed. We will try to evaluate equation (5) to order $x$, where $x<10 \%$. Since the increase of internal energy of the solution with $T$ at fixed $x$ is the same within experimental accuracy as that for an ideal Fermi gas, one has

$$
\Delta \mu_{3}(T, x)=\Delta \mu_{3}\left(T / T_{F}\right) .
$$

where $T_{F}$, the Fermi temperature, is a function of $x$ given by

$$
T_{F}=\frac{p_{F}^{2}}{2 m^{*} k}=\frac{\hbar^{2}}{2 m^{*} k}\left(\frac{3 \pi^{2}}{\omega(x)}\right)^{2 / 3} x^{2 / 3} .
$$

Here $p_{F}$ is the Fermi momentum, $k$ is Boltzmann's constant, $\omega(x)=\Omega /\left(N_{3}+N_{4}\right)$ is the average volume per helium atom, and $m^{*}$ depends weakly on $x$. In the formula for $\omega, \Omega$ is the total volume, $N_{3}$ is the number of $\mathrm{He}^{3}$ atoms, and $N_{4}$ is the number of $\mathrm{He}^{4}$ atoms. For small $x$ the quantity $\omega(x)$ is determined experimentally to be

$$
\omega(x)=\omega_{0,4}(1+\alpha x) \text {. }
$$

where $\omega_{0,4}$ corresponds to a molar volume in pure $\mathrm{He}^{4}$ of $27.5 \mathrm{~cm}^{3} / \mathrm{mole}$ and $\alpha$ is a parameter approximately equal to $0.3[16,17,22]$. In the vicinity of $x=6.35 \%, T_{F}$ in equation (6) depends approximately on $x$ by $T_{F}=2.38 \mathrm{~K}^{0} x^{2 / 3}$. Using this value for $T_{F}, \Delta \mu_{3}$ may then be 
conveniently obtained (at least for values of $x$ near the above) from the equation

$$
\frac{\Delta \mu_{3}}{k T_{F}}=\frac{T}{T_{F}} \eta\left(\frac{T}{T_{F}}\right)-1 \text {. }
$$

where $\eta\left(\frac{\Gamma}{T_{F}}\right)$ is tabulated in Table 8.1 of McDongall and Stoner [14]. Parenthetically, it is superficially surprising that $\Delta \mu_{3}=\left(\frac{\partial \Delta F}{\partial N_{3}}\right)_{T, \Omega, N_{4}}$ [25] is the same as the change in chemical potential for an ideal gas. Here $\Delta F=\Delta U-T \Delta S$, where $\Delta U$ and $\Delta S$ are the experimentally determined changes from $T=0$ at fixed $N_{3}, V_{4}$ of the internal energy and entropy of the entire solution for $P \cong 0$. However, to accuracy $x$, one has $\Omega=v_{4} \omega_{0,4}$, so that holding $\Omega$ constant is the same as holding $\mathrm{V}_{4}$ constant in the derivative. Referring to values given in Appendix $A$, at low $T$ $\Delta U=N_{3} k \frac{\pi^{2}}{4} \frac{T^{2}}{T_{F}}$ and $T \Delta S=N_{3} k \frac{\pi^{2}}{2} \frac{T^{2}}{T_{F}}$ so that $\Delta_{i}=-N_{3} k \frac{\pi^{2}}{4} \frac{\Gamma^{2}}{T_{F}}$. Approximating $I_{F}^{\prime}$ by $T_{F}=\gamma\left(V_{3} / N_{4}\right)^{2 / 3}$, equation (7), with $\gamma$ constant one finds $\Delta \mu_{3}=\left(\frac{\partial \Delta F}{\partial V_{3}}\right)_{T, N_{4}}=$ - $k\left(\pi^{2} / 12\right) T^{2} / T_{F}$, which is the low tenperature approximation to the change in chemical potential of the ideal Fermi gas.

The quantity $\mu_{3_{d}}(0, x)$ in equation (5) cannot be evaluated with as much confidence, experimentally, as $\Delta \mu_{3}(T, x)$. Both the phase separation curve and the osmotic pressure (to be discussed later) depend on $\mu_{3}(0, x)$, but the former is not sufficiently sensitive to the $x$-dependence of $\mu_{3}(0, x)$ while the latter has not been measured at low enough temperatures [26]. We have therefore used a calculation [27] based on the BBP theory [15], on work by Ebner [28], and on experimental measurements of transport coefficients $[12,29]$. The result is

$$
\mu_{3_{d}}(0, x)=-E_{0_{3}}+\frac{p_{F} 2}{2 m}-x \alpha^{2} m_{4} c^{2}\left[1-\frac{3}{4 p_{F} 3} \int_{0}^{2 p_{F}} q^{2}\left(1-\frac{q}{2 p_{F}}\right)[V(q) / V(0)] d q\right] \text {. }
$$

The first term on the right side is the binding energy of a single $\mathrm{He}^{3}$ quasiparticle to the superfluid (compare equation 1 ). The second term is the kinetic energy $k T_{F}$ (with $m=2.34 \mathrm{~m}_{3}$ [15]) which must be added to put a particle on the Fermi surface. The third term is a correction to the binding energy resulting from the mutual interaction of the $\mathrm{He}^{3}$ quasiparticles. The quantity $-x \alpha^{2} m_{4} c^{2}$ comes from the result $[15,30]$ that two He 3 quasiparticles of opposite spin attract one another on the average with the energy $-\alpha^{2} m_{4} c^{2} / V_{4}$, where $\alpha$ is defined in equation (8), $m_{4}$ is the atomic mass of $\mathrm{He}^{4}$, and $c$ is the hydrodynamic sound velocity in pure $\mathrm{He}^{4}$. The term involving the integral arises from the possibility of exchange scattering of particles of like spin. The BBP effective potential $V(q)$ enters here. In view of all the experimental and theoretical uncertainties we have elected to use the power law form of $V(q)$ advocated in Ref.29. The resulting explicit form for $\mu_{3}(0, x)$ is

$$
\frac{\mu_{3 d}(0, x)}{k}=-\frac{E_{0}}{k}+2.58 \mathrm{~K}^{\circ} x^{2 / 3}-2.74 \mathrm{~K}^{0} x\left[1 / 2+4.65 x^{2 / 3}-11.0 x^{4 / 3}\right] .
$$


Putting equations (9) and (10a) in equation (5) $\mu_{3}$ is determined, at least approximately. The right side of equation (3) is given by

$$
\frac{\mu_{3}}{k}=-\frac{L_{0_{3}}}{k}-\frac{1}{k} \int_{0}^{T} s_{3_{c}}\left(T^{\prime}\right) d T^{\prime},
$$

where $L_{0_{3}}$ is the binding energy at $T=0, P=0$ of a $\mathrm{He}^{3}$ atom to pure liquid He ${ }^{3}$. To evaluate equation (11) we use heat capacity data reviewed by Wheatley [31]. Since $E_{0_{3}}-L_{0_{3}}$ is not known from other data, equation (3), which relates the phase separation curve to $\mu_{3}(0, x)$, allows $E_{0_{3}}-L_{0_{3}}$ to be determined for a given $\mathrm{He}^{3}$ quasiparticle mutual interaction. However, the phase separation curve is not very sensitive to how the energy is divided between the mutual interaction of $\mathrm{He}^{3}$ quasiparticles and $E_{0_{3}}-L_{0_{3}}$.

Designing the dilution refrigerator and understanding its characteristics require a knowledge of the coefficients of viscosity and thermal conductivity for both pure $\mathrm{He}^{3}$ and dilute solutions of $\mathrm{He}^{3}$ in $\mathrm{He}^{4}$. In the case of pure $\mathrm{He}^{3}$ we will use a limiting low-temperature viscosity given by the value deduced [32] from ultrasonic attenuation measurements,

$$
\eta_{c} T^{2}=2 \times 10^{-6} \mathrm{dyn} \sec \mathrm{K}^{\circ 2} / \mathrm{cm}^{2} \text {. }
$$

The viscosity at higher temperatures is needed for impedance design work and may be found in Ref. 31. The thermal conductivity is given in the limit of low temperatures by

$$
{ }_{\kappa_{c}} T=33 \frac{\mathrm{erg}}{\mathrm{sec} \mathrm{cm}}\left[1-T / 0.54^{\circ} \mathrm{K}\right]^{-1}
$$

For the dilute solutions it is the limiting low-temperature viscosity of a nearly saturated solution $(x=0.063)$ which is needed. Although low-temperature flow measurements of viscosity have never been made, the ultrasonic attenuation is intimately connected with viscosity. Measurements of ultrasonic attenuation by Abraham and co-workers [33] are in good agreement with the theory of Bayin and Ebner [34]. In the comparison of experiment and theory, the empirical low-temperatúre relaxation time for viscosity is rather similar to that calculated by Roach [35]. Hence for design purposes we use the limiting low-temperature viscosity calculated by Roach using the BBP potential. The result for $x=0.063$ is

$$
\eta_{d} T^{2}=5 \times 10^{-7} \mathrm{dyn} \sec \mathrm{K}^{\circ 2} / \mathrm{cm}^{2} \text {, }
$$

The thermal conductivity of dilute solutions has very interesting characteristics. Low-temperature measurements of Ref. 29 are shown in Fig. 3. At sufficiently low temperatures $\kappa_{d} T$ is constant, at least approximately. For $x=0.063$ we will assume that

$$
\mathrm{k}_{d} T=30 \mathrm{erg} / \mathrm{sec} \mathrm{cm}
$$

is accurate enough to give an adequate description of the properties of the dilution refrigerator. At higher T, Fig. 3, the thermal conductivity rises again due to $\mathrm{He}^{4}$ phonon conduction. Accurate measurements of thermal conductivity in dilute solutions at higher temperatures, but below $0.7^{\circ} \mathrm{K}$ are not available, excepting a few measurements of Ptukha [36] which extend down to about $0.65^{\circ} \mathrm{K}$. However, Baym and Ebner [34] have computed the phonon thermal conductivity over the temperature range of interest $\left(\sim 0.1^{\circ} \mathrm{K}-0.8^{\circ} \mathrm{K}\right)$. Their calculations are in reasonable agreement with both Ptukha's measurements and some preliminary measurements of Abel, Johnson, 
Wheatley, and Zimmermann. The conductivity rises slowly as $T$ decreases in the above range, but for design purposes it is adequate (within a factor 2) to take $\kappa_{d}$ constant at

$$
\mathrm{K}_{d} \approx 2 \times 10^{4}\left(\frac{0.01}{x}\right) \mathrm{erg} / \mathrm{sec} \mathrm{cm} \mathrm{K}{ }^{\circ} \quad\left(0.8^{\circ} \mathrm{K}>T>0.1^{\circ} \mathrm{K}\right) .
$$

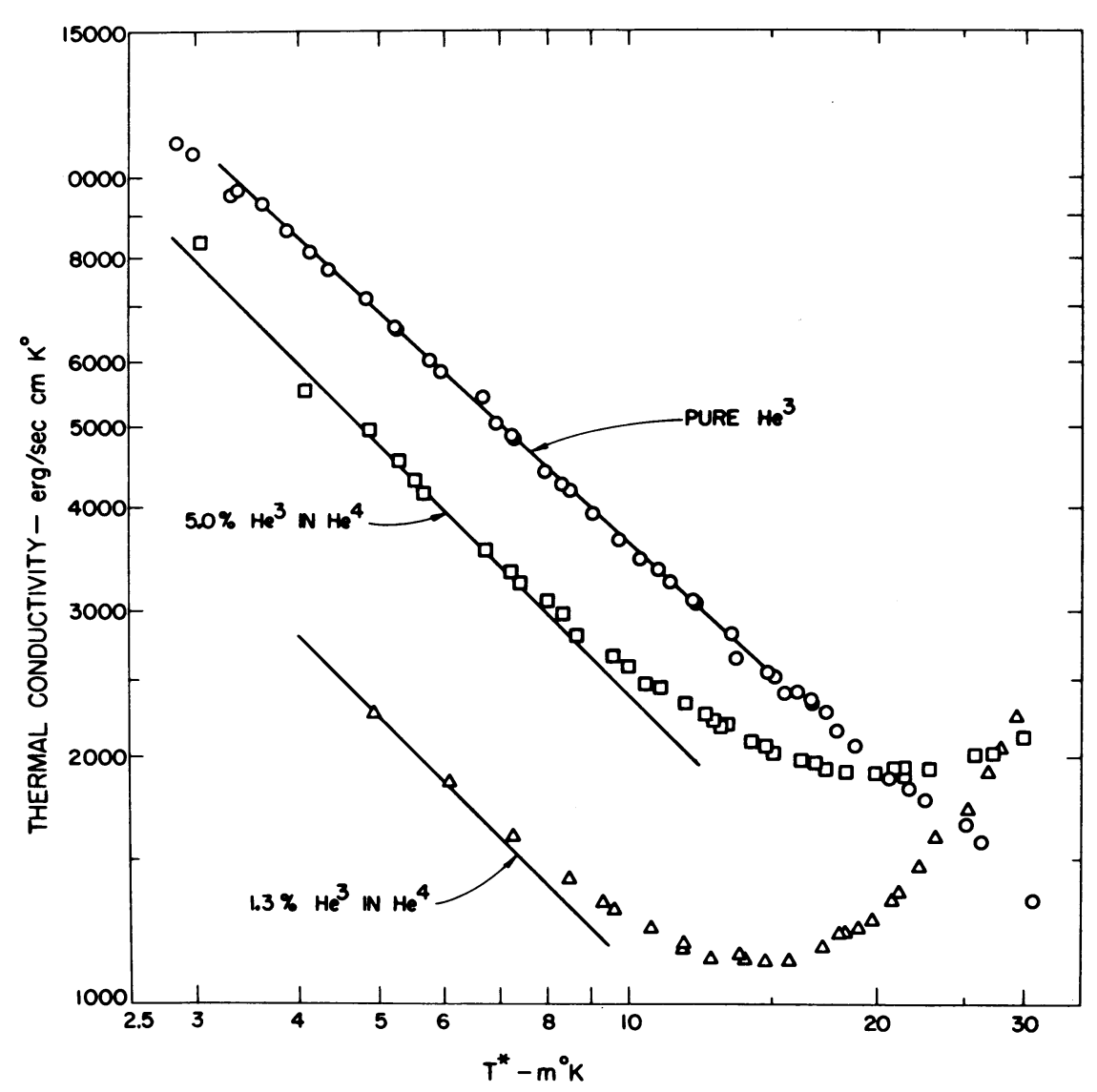

FIGURE 3

Thermal conductivity coefficient for dilute solutions of $\mathrm{He}^{3}$ in $\mathrm{He}^{4}$ of nominal concentrations $1.3 \%$ and $5.0 \%$ and at saturated vapor pressure. The thermal conductivity of pure $\mathrm{He}^{3}$ at saturated vapor pressure is shown for comparison.

\section{The Dilution Process}

Refrigeration in the dilution refregerator is produced in a container called the mixing chamber. Under steady-state conditions in an "evaporation" type refrigerator the temperature of 
the helium in the mixing chamber is constant while $\mathrm{He}^{3}$ from the concentrated (upper) phase dissolves in the superfluid dilute phase at a constant rate. Throughout the dilute phase, as indicated in Section II, steady-state conditions require $\mu_{4}$ to be constant. The pressure is also very nearly zero under normal operating conditions. Hence in the thermodynamic analysis it is required that the $\mathrm{He}^{3}$ dissolve in the dilute solution at constant (zero) pressure and constant $\mu_{4}$. In a continuously onerating dilution refrigerator concentrated $\mathrm{He}^{3}$ is supplied, under steady-state conditions, at the same rate as it dissolves. In a "single-cycle" refrigerator no new $\mathrm{He}^{3}$ is added, and the quantity of $\mathrm{He}^{3}$ in the upper phase is gradually depleted.

In Fig. 4 is a diagram illustrating the thermodynamics of the mixing chamber of a continuously operating dilution refrigerator. In order to deal with a fixed amount of $\mathrm{He}^{3}$ and $\mathrm{He}^{4}$

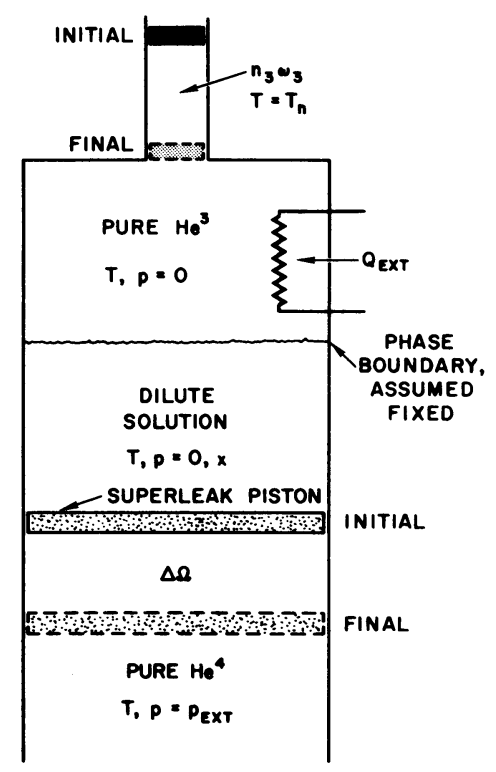

FIGURE 4

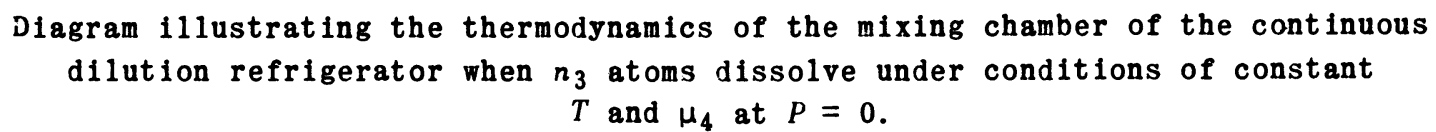

while satisfying the requirements that $\mu_{4}$ be constant and $P$ zero, imagine that at an arbitrary place in the dilute solution a "superleak piston" is located and all $\mathrm{He}^{3}$ below it removed. The superleak piston is made of such fine-grained, tightly-packed powder that only superfluid helium can pass it. In order for $\mu_{4}$ to be the same on both sides of the piston, the pure He 4 below the piston must be in tension (as actually occurred in some recent experiments of Wilson, Edwards and Tough) [26]. This follows from equating $\mu_{4}$ above and below the piston using $d \mu_{0,4}=$ $-s_{0,4} d T+\omega_{0,4} d P$, where $s_{0,4}$ and $\omega_{0,4}$ are the entropy and volume per atom of pure He ${ }^{4}$ to 
evaluate $\mu_{4}$ for pure $\mathrm{He}^{4}$ and equation (2) to evaluate the effect of $\mathrm{He}^{3}$ on $\mu_{4}$. Letting $\mu_{0,4}^{0}$ be the value of $\mu_{4}$ for pure $\mathrm{He}^{4}$ at $T=0, P=0$ one has

$$
\mu_{0,4}^{\circ}-\int_{0}^{T} s_{0,4} d T-\int_{0}^{x} \frac{x}{1-x}\left[\frac{\partial}{\partial x} \mu_{3 d}(T, x)\right] d x=\mu_{0,4}^{\circ}-\int_{0}^{T} s_{0,4} d T+\omega_{0,4} P_{\mathrm{ext}} \text {. }
$$

From this it follows that

$$
P_{\text {ext }}=-\frac{1}{\omega_{0,4}} \int_{0}^{x} \frac{x}{1-x}\left\lfloor\frac{\partial}{\partial x} \mu_{3 d}(T, x)\right\rfloor d x
$$

The pressure drop which occurs across the superleak piston is the familiar osmotic pressure drop which develops in equilibrium across a semipermeable membrane. Explicit substitution of the high temperature expression for $\mu_{3}$, neglecting the effective interactions between $\mathrm{He}^{3}$ quasiparticles, in equation (18) leads in the limit $x<1$ to

$$
\left(-P_{\text {ext }}\right) \frac{\omega_{0,4}}{x}=k T .
$$

Since $\omega_{0,4} / x$ is the average volume per atom of $\mathrm{He}^{3}$, the osmotic pressure drop is the same as the pressure exerted by an ideal Boltzmann gas of $\mathrm{He}^{3}$ particles. The condition $\mu_{4}=$ constant is equivalent to $P_{\text {ext }}(T, x)=$ constant. Under suitable conditions [equation (19) valid] the pressure on the piston which must be resisted is just that of an ideal gas of $\mathrm{He}^{3}$ particles. This gives us a fruitful, though not precise, way of thinking about processes which occur in the dilution refrigerator.

Concentrated liquid $\mathrm{He}^{3}$ enters the mixing chamber at nearly zero pressure and at a temperature $T_{n}$ which is made as low as possible by means of heat exchangers utilizing the refrigeration in the effluent $\mathrm{He}^{3}$. Since under steady-state conditions just as much $\mathrm{He}^{3}$ enters the mixing chamber per unit time as leaves it, the phase boundary between the upper and lower phases remains fixed in space.

For the sake of definiteness imagine an initial configuration of pistons as indicated in Fig. 4. The temperature is low enough, Fig. 2, so that the upper phase may be assumed to be pure $\mathrm{He}^{3}$. The upper piston sweeps $n_{3}$ atoms of pure $\mathrm{He}^{3}$ at temperature $T_{n}$ into the mixing chamber, $n_{3}$ atoms of $\mathrm{He}^{3}$ dissolve in superfluid dilute solution, the superleak piston sweeps out volume $\Delta \Omega$ such that $x$ remains constant, $n_{4}$ atoms of $\mathrm{He}^{4}$ pass through the superleak piston (again to keep $x$ constant), and heat $Q_{\text {ext }}$ is added from external sources to keep $T$ constant. Details of the thermodynamics are given in Ref. 11. One sets the heat $Q_{\text {ext }}$ equal to the change of internal energy of the helium plus the work done by the helium in moving the superleak piston. Simplification of the resulting equation may be made by using equation (3) in the form $u_{3_{d}}-T_{s_{3_{d}}}=$ $u_{3_{c}}-T s_{3_{c}}(P=0)$ and the constancy of $\mu_{4}$ across the superleak piston in the form $\mu_{4_{d}}=$ $u_{4_{d}}-T_{s_{4_{d}}}=u_{0,4}+\omega_{0,4} P_{\text {ext }}$. (One sets $\mu_{0,4}=u_{0,4}$.) The entropy of pure He ${ }^{4}$ is negligible in the temperature range of interest. The result is

$$
Q_{\text {ext }}+n_{3}\left[u_{3_{c}}\left(T_{n}\right)-u_{3_{c}}(T)\right]=T\left\{\left[n_{3} s_{3_{d}}(T, x)+n_{4} s_{4_{d}}(T, x)\right]-n_{3} s_{3_{c}}(T)\right\} \text {. }
$$


The quantity in square brackets on the left side is the change in the internal energy per atom of pure $\mathrm{He}^{3}$ as it cools from $T_{n}$ to $T$. The quantity in square brackets on the right is the standard form relating a mean quantity to a partial quantity in thermodynamics. That is, one has

$$
n s_{d}(T, x)=n_{3} s_{3}(T, x)+n_{4} s_{4}(1, x) \text {. }
$$

where $n=n_{3}+n_{4}, s_{d}(T, x)$ is the mean entropy per atom in the solution, $s_{3_{d}}$ is the partial entropy of the solution per atom of $\mathrm{He}^{3}$, and $s_{4_{d}}$ is the partial entropy of the solution per atom of $\mathrm{He}^{4}$ [37]. It is the quantity ${ }^{2} s_{d}$ which is measured in a calorimetric experiment. Hence the most convenient form for equation (20) is

$$
2_{\text {ext }}+n_{3}\left[u_{3_{c}}\left(T_{n}\right)-u_{3_{c}}(T)\right]=T\left[n s_{d}(T, x(T))-n_{3} s_{3_{c}}(T)\right] \text {. }
$$

where $s_{3_{c}}$ is the entropy of pure $\mathrm{He}^{3}$ per atom and $x(T)$ is given by the solution of equation (3) - the left branch of the phase separation curve. Hence equation (22) depends on $T$ only.

The mixing chamber of the single-cycle dilution refrigerator is similar to that of the continuously operating dilution refrigerator with the exception that no inlet for concentrated $\mathrm{He}^{3}$ is provided. Figure 4 thus is suitable for describing the thermodynamical analysis provided the upper piston is removed. The result of the thermodynamical analysis [11] is that the heat $\eta_{\text {ext }}$ which must be added to keep $T$ constant while $n_{3}$ atoms of $\mathrm{He}^{3}$ dissolve in the dilute solution at constant $\mu_{4}$ and zero pressure is given by

$$
Q_{\text {ext }}=T\left[n s_{d}\left(T, x\left(T^{\prime}\right)\right)-n_{3} s_{3}(T)\right] .
$$

The meaning of the symbols is the same as in equation (22). Equation (23) differs from equation (22) only in that the term in $u_{3}$ on the left side is absent. Both equations are quite reasonable. The heat added in the continuous refrigerator is the sum of that added from external sources and that given up by the incoming $\mathrm{He}^{3}$ as it cools to the temperature of the mixing chamber. In the single-cycle refrigerator the second term is absent. The right side of equations (22) and (23) is just a $T \Delta s$ term similar to that found in more familiar systems.

Numerical evaluation of equations (22) and (23) can proceed once $x(T)$, the phase separation curve, is known. The phase separation curve has now been measured with some precision by Ifft et al. [22]. However, when we were designing the dilution refrigerator these measurements had not been completed, so we based our numerical analysis on a measurement by Vilches and Wheatley [10] of the right side of equation (23) in an experiment with the single-cycle dilution refrigerator. It was found that the temperature of the mixing chamber remained constant at $T$ when $\mathrm{He}^{3}$ was being removed at rate $\dot{\mathrm{n}}_{3}$ if heat were supplied at the rate

$$
\dot{Q}=\dot{n}_{3} k T^{2} 10 \mathrm{~K}^{\circ-1} \text {. }
$$

where the numeric has an accuracy of about 5\%. From Appendix A and the properties of pure $\mathrm{He}^{3}$, a low-temperature approximation to equation (23) is

$$
\dot{Q}_{\text {ext }}=\dot{n}_{3} k T^{2}\left[\frac{\pi^{2}}{2 T_{F}}-\Gamma\right] \text {. }
$$

where $\Gamma=3.0 \mathrm{~K}^{0^{-1}}$. Comparing equations (24) and (25) we find $T_{F}=0.379^{\circ} \mathrm{K}$. Using data given in 
Section II, this value of $T_{F}$ corresponds to a limiting solubility $x_{0}$ of $\mathrm{He}^{3}$ in He $\mathrm{H}^{4}$ at $T=0$ of 0.0635 , within the range of probability of the value, $(0.0637 \pm 0.0005)$, given in Ref. 22 . Using our value of $x_{0}$ in equations (10) and (11) we find $\left(E_{0_{3}}-L_{0_{3}}\right) / k=0.242^{\circ} \mathrm{K}$. The left branch of the phase separation curve was then obtained using equations (5) and $(9-11)$ to solve equation (3). The result, shown on Fig. 5 , is in good agreement with the form measured by Ifft et al. [22] to be

$$
x=x_{0}\left[1+10.8 \mathrm{~K}^{\circ-2} T^{2}\right] \quad T<0.15^{\circ} \mathrm{K}
$$

We therefore can evaluate the right side of equations (22) and (23) with some confidence. In Fig. 6 we have plotted a related but normalized quantity given by

$$
\frac{T \Delta s(T)}{k} \equiv T\left(\frac{n s_{d}(T)}{n_{3} k}-\frac{s_{3}(T)}{k}\right) .
$$

From equation (24) this quantity is $10 \mathrm{~K}^{-1} T^{2}$ at low temperatures. The term $u_{3_{c}}\left(T_{n}\right)-u_{3_{c}}(T)$ in equation (20) may be obtained from Fig. 7. At low enough temperatures $u_{3}(T)$ is given with sufficient accuracy by

$$
\frac{u_{3_{c}}(T)}{k}=1.5 \mathrm{~K}^{0^{-1}} T^{2} \text { (low temperature). }
$$

The dilution process illustrated schematically in Fig. 4 is not the only way of diluting $\mathrm{He}^{3}$ or a dilute solution of $\mathrm{He}^{3}$ in $\mathrm{He}^{4}$ reversibly with superfluid $\mathrm{He}^{4}$. An alternative method suggested by Fig. 4 and its analysis is based on the use of an actual superleak, rather than the superleak piston of Fig. 4, which is introduced as a constraint to facilitate the thermodynamical analysis. Devices using a superleak so that $\mathrm{He}^{4}$ flows instead of $\mathrm{He}^{3}$ will be called "superleak operated" devices. We have not yet built devices of this type, but some work along these lines has been begun by de Bruyn Ouboter and Taconis [39]. Since they will no doubt be of importance in the future we will discuss them here. Those which we have considered are all of the single-cycle type. The limiting low-temperature behaviour of such devices may be quite different from that of the devices already discussed. In superleak-operated devices the mixing chamber is only partially filled with $\mathrm{He}^{3}$ and $\mathrm{He}^{4}$. It is connected by means of a superleak to another chamber containing pure $\mathrm{He}^{4}$ which may be at the same or a different temperature as the mixing chamber. Dilution is caused to take place reversibly by movement of superfluid $\mathrm{He}^{4}$ into the mixing chamber rather than by movement of $\mathrm{He}^{3}$ out of the mixing chamber as in devices previously considered.

It is important to emphasize that a superleain is not the same as a semipermeable membrane. A superleak is typically either a tube packed tightly with very finely grained powder or a cylinder of porous Vycor glass. Superfluid $\mathrm{He}^{4}$ flows without dissipation through a superleak, though its kinetic energy is dissipated in the region where it discharges. If there is a difference in pressure (osmotic pressure difference) across the superleak, then $\mathrm{He}^{3}$ quasiparticles will also flow through the superleak. Their flow, however, is inhibited by viscosity. If the channels in the superleak are long enough and narrow enough the flow impedance is so high that even in the duration of an entire experiment no normal fluid will pass. Any tube connecting two vessels containing superfluid and $\mathrm{He}^{3}$ quasiparticles is a "superleak" in that an osmotic pressure difference, in the sense of the present article, may be developed across it which may be relaxed in a time determined by the viscous flow of the $\mathrm{He}^{3}$ through the tube. If the tube is hollow, the time 
of relaxation may be relatively short. If the tube is packed with powder, this time will be longer. The concept of a superleak is thus a relative one. For superleak operated dilution cooling devices the above time of relaxation should be long compared with the length of time the device is to be operated. As a practical matter, however, it should be possible to compensate for small amounts of $\mathrm{He}^{3}$ which pass the superleak.

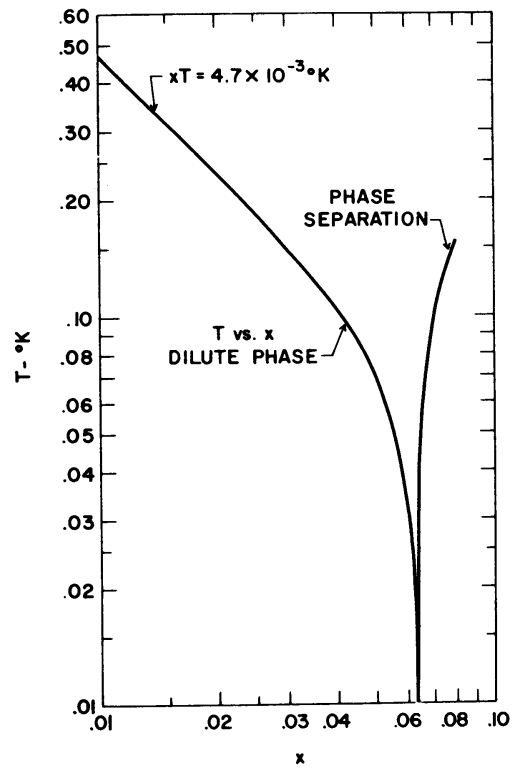

FIGURE 5

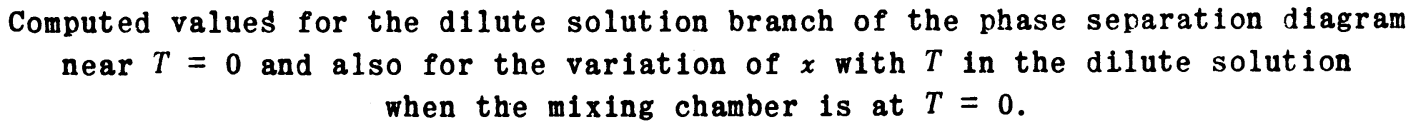

The thermodynamic analysis of a superleak-operated device is facilitated by considering the diagram shown in Fig. 8. In the actual device $n_{4}$ atoms of superfluid enter the mixing chamber, in this case containing only dilute solution, and heat $\rho_{\text {ext }}$ is added to keep the temperature constant. The physical process is analogous to the expansion of a gas in which heat may be added at constant $T$ while work is being done by the gas. To synthesize the actual process, we imagine in Fig. 4 that the dilute solution is separated from pure $\mathrm{He}^{4}$ by a superleak piston as shown. The $n_{4}$ atoms of $\mathrm{He}^{4}$ may be added reversibly by allowing the superleak piston to sweep out volume $\Delta \Omega=n_{4} \omega_{0,4}$ (equation 8). Again $P_{\text {ext }}$ is negative, equations (18) and (19), while the solution is at saturated vapor pressure, which is essentially zero pressure for the temperatures of interest. The thermodynamic analysis is quite similar to that given for the continuously-operating refrigerator. The result is

$$
Q_{\text {ext }}=n_{4} T s_{4_{i}},
$$


where

$$
s_{4 d}=\left(\frac{\partial S_{d}}{\partial N_{4}}\right)_{T, P=0, N_{3}}
$$

and $S_{d}$ is the entropy of the whole dilute solution. The partial entropy $s_{4 d}$ may be obtained conveniently from the measured mean entropy of the solution $s_{d}=S_{d} /\left(V_{3}+N_{4}\right)$ by the equation

$$
s_{4_{d}}=s_{d}-x\left(\frac{\partial s_{d}}{\partial x}\right)_{T, P=0}
$$

It is also possible for the mixing chamber in a superleak operated device to contain pure $\mathrm{He}^{3}$ in equilibrium with a saturated solution of $\mathrm{He}^{3}$ in superfluid He${ }^{4}$. Figure 8 would then be modified to show pure $\mathrm{He}^{3}$ floating on top of the dilute solution. The result of the thermodynamic analysis gives equation (23) if $T$ is held constant by adding heat ?ext while $n_{4}$ atoms

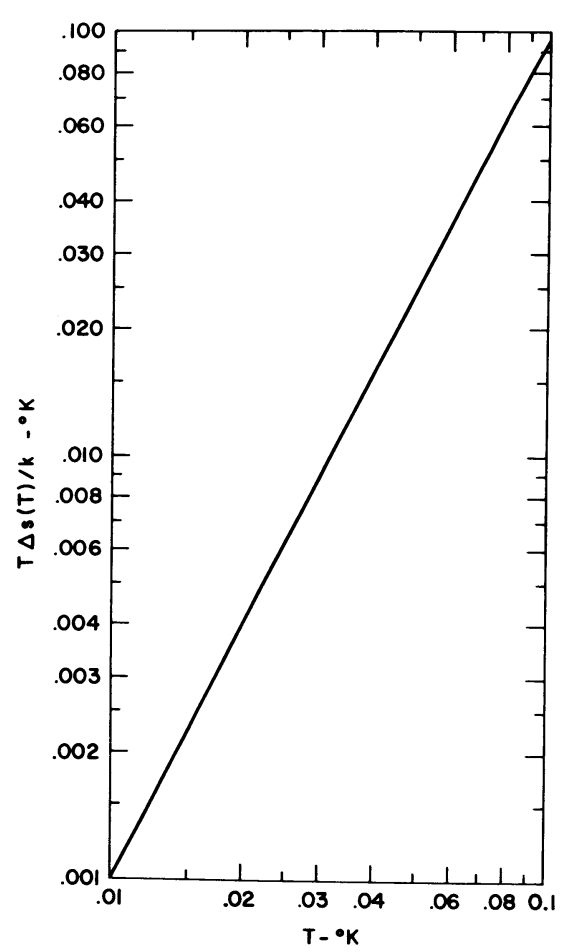

FIGURE 6

Refrigeration capability $T \Delta s(T) / k=T\left[n s_{d}(T)-n_{3} s_{3_{c}}(T)\right] / n_{3} k$ of the single-cycle dilution refrigerator as a function of temperature. 
of $\mathrm{He}^{4}$ enter through the superleak and $n_{3}$ atoms of $\mathrm{He}^{3}$ are dissolved in order to maintain the two-phase equilibrium. This result serves to emphasize the operational difference between the two types of devices. To get the same amount of refrigeration, approximately $x^{-1}$ times as much $\mathrm{He}^{4}$ must flow in a superleak-operated device as in a $\mathrm{He}^{3}$ flow operated device. For the same total cooling capacity the initial amount of $\mathrm{He}^{3}$ should be the same. In the $\mathrm{He}^{3}$ flow operated device the volume of mixing chamber required is just the volume $N_{3} \omega_{0,3}$ of the $\mathrm{He}^{3}$. In the superleak-operated device the volume required is $\left(\omega_{0,4} / x \omega_{0,3}\right)$ times larger, or about 12 times larger for $x=6.3 \%$. There are other important differences, also. There is refrigeration available in the $\mathrm{He}^{3}$ stream leaving the $\mathrm{He}^{3}$ flow device which is not available in the superleak device. However, the $\mathrm{He}^{3}$ flow results in an intrinsic viscous heating of the mixing chamber which does not seem to occur in the superleak device.

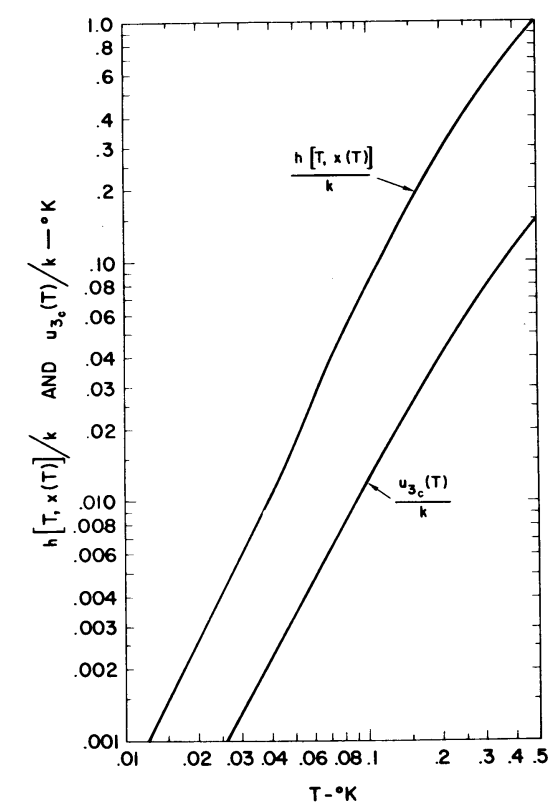

FIGURE 7

The quantities $u_{3} / k$ and $h / k$ [equation (44)] as functions of temperature.

If a $\mathrm{He}^{3}$ flow-type, single-cycle device is not supplied heat while $\mathrm{He}^{3}$ atoms are removed, then the helium which remains will be cooled. Supposing that the mixing chamber contains mostly pure $\mathrm{He}^{3}$, then $\dot{Q}_{\text {ext }}$ is replaced by the heat capacity of the $\mathrm{He}^{3}$ in the mixing chamber times the rate of change of temperature. This leads at low temperatures to

$$
\frac{\Delta T}{T}=\frac{1}{\Gamma}\left[\frac{\pi^{2}}{2 T_{F}}-\Gamma\right] \frac{\Delta V_{3}}{V_{3}} \cong 3.3 \frac{\Delta N_{3}}{N_{3}},
$$

where $\Gamma$ is the heat capacity coefficient in pure $\mathrm{He}^{3}\left(3.0 \mathrm{~K}^{-1}\right)$, the quantity in square brackets 
is $10 \mathrm{~K}^{\mathrm{O}^{-1}}$, equation (24), and $\Delta \mathrm{V}_{3} / \mathrm{N}_{3}$ is the fractional change in the amount of He ${ }^{3}$ within the mixing chamber. A similar analysis may be made for the case of the superleak-operated device containing only dilute solution in the mixing chamber. Using Appendix A to evaluate equation (29) at low temperatures, we find for a dynamic arrangement in which $\mathrm{He}^{4}$ is added at a constant rate $\dot{n}_{4}$

$$
\dot{\mathrm{Q}}_{\mathrm{ext}}=\dot{\mathrm{n}}_{4} k \frac{\pi^{2}}{3} \frac{T^{2}}{T_{F}} x .
$$

If heat is not supplied externally and the temperature is allowed to change, we find

$$
\frac{\Delta T}{T}=-\frac{2}{3} \frac{\Delta N_{4}}{N_{4}} .
$$

where $\Delta N_{4} / N_{4}$ is the fractional change in the amount of He $\mathrm{H}^{4}$ within the mixing chamber. Comparison of equations (32) and (34) shows that the $\mathrm{He}^{3}$ flow-type device containing two phases in equilibrium is much more effective in self-cooling by "evaporation" than the superleak operated

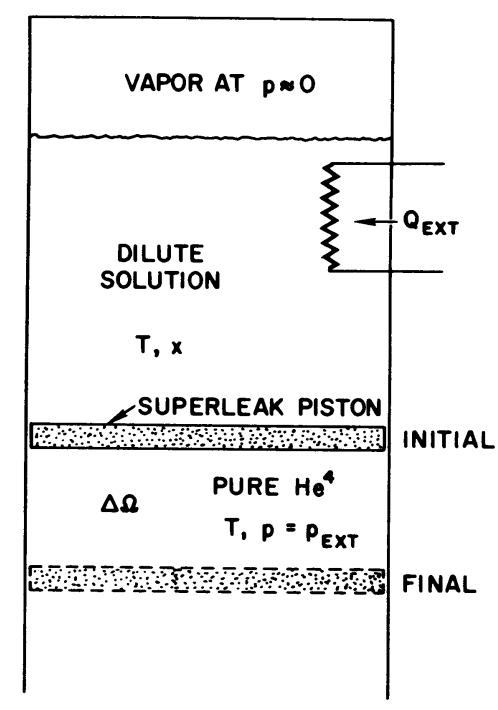

FIGURE 8

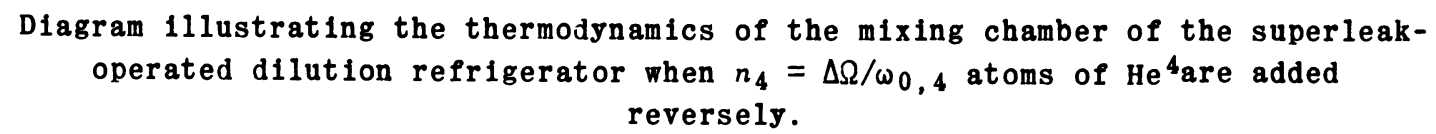

device, which is self-cooled by the analog of adiabatic expansion. The superleak operated device in which there are two phases in equilibrium in the mixing chamber will also have inferior characteristics relative to equation (32) since the amount of $\mathrm{He}^{3}$ in the mixing chamber is not 
depleted in the dilution process while the mean heat capacity per particle is increased.

In the superleak-operated device it is necessary to provide on the side of the superleak away from the mixing chamber a value of $\mu_{4}$ which is less than $\mu_{0,4}^{\circ}$ by an amount given by the left side of equation (17). Conceptually, the easiest way to do this is to let the $\mathrm{He}^{4}$ on the side of the superleak away from the mixing chamber be under tension, as was achieved experimentally by Wilson, Edwards and Tough [26]. Whether or not the quantity of $\mathrm{He}^{4}$ under tension may be varied, as is necessary in a device used to produce refrigeration, has not been studied experimentally. Equation (17) may be generalized somewhat if the $T$ limits of the entropy integrals on the left and right sides are allowed to be different. The $T$ limit on the left side is $T_{M C}$, so this entropy integral is negligible compared with the term in $x$. However, the $T$ limit on the right side may be made anything which is experimentally useful. It is in fact just $\omega_{0,4}$ times the fountain pressure. Thus equilibrium could be maintained across the superleak by letting the entropy integral on the right side at zero pressure balance the $x$ integral on the left. This may be described loosely as letting the fountain pressure balance the osmotic pressure. There would be no pressure drop across the superleak and hence no osmotic pressure difference in the usual sense of the word "osmotic". Equation (17) may be modified further by admitting a term in $x$ on the right side. It is then evident that the equilibrium across the superleak may be achieved if the sum $P-P_{\text {osmotic }}-P_{\text {fountain }}$ is the same on both sides, where $P$ is the mechanical pressure and

$$
\omega_{0,4} P_{\text {osmotic }}=\int_{0}^{x} \frac{x}{1-x}\left[\frac{\partial}{\partial x} \mu_{3}(T, x)\right] d x \text {. }
$$

and

$$
\omega_{0,4} p_{\text {fountain }}=\int_{0 .}^{T} s_{0,4}(T) d T .
$$

\section{Enthalpy}

In the continuously' operating dilution refrigerator the $\mathrm{He}^{3}$ which leaves the mixing chamber in the dilute phase may be used to precool the incoming concentrated $\mathrm{He}^{3}$. The flowing $\mathrm{He}^{3}$ is also heated due to the effect of viscosity. It is therefore useful to estimate the cooling power of $\mathrm{He}^{3}$ in the dilute phase. The concentrated phase flows at nearly zero pressure. The enthalpy of pure $\mathrm{He}^{3}$ is thus just the internal energy of pure $\mathrm{He}^{3}$, which has already been given on Fig. 7. The concentrated phase also contains $\mathrm{He}^{4}$ as a result of imperfections in the method of circulating $\mathrm{He}^{3}$. The $\mathrm{He}^{4}$ has a harmful effect at high temperatures, but below $0.1^{\circ} \mathrm{K}$ its effect is small. Effects of $\mathrm{He}^{4}$ will be discussed separately.

The $\mathrm{He}^{3}$ concentration varies with $T$ in the dilute phase. This must first be calculated. In the dilute phase $\mathrm{He}^{3}$ flows as a result of gradients in the real osmotic pressure. For the present calculation we will assume that these gradients are small so that the condition $\mu_{4}=$ constant is imposed for a constant, and essentially zero, pressure throughout the dilute phase. We will also neglect any contribution of $\mathrm{He}^{4}$ entropy to $\mu_{4}$. The equation $\mu_{4}=$ constant contains only $T$ and $x$ as variables, so it determines the variation of concentration with temperature throughout the dilute phase once the concentration at one temperature is known. For present purposes we will assume that the mixing chamber is very cold indeed. In this case we can take

$$
\mu_{4}(T, x)=\mu_{4}\left(0, x_{0}\right) \text {. }
$$


where $x_{0}$ (taken to be 0.0635 ) is the concentration of the saturated solution at $T=0$. We have solved equation (37) numerically. We first write

$$
\mu_{4}(T, x)=\mu_{0,4}^{0}-\int_{0}^{x} x\left(\frac{\partial \mu_{3} d}{\partial x}\right)_{T} d x
$$

from equation (2) (approximating $1-x \approx 1$ ), and then separate $\mu_{4_{d}}$ into two parts,

$$
\mu_{4}(T, x)=\mu_{4}(0, x)+\Delta \mu_{4}(T, x) .
$$

The quantity $\mu_{4}(0, x)$ is obtained by substituting equation (10a) in (38) and integrating. The quantity $\Delta \mu_{4_{d}}(T, x)$ is obtained by using the ideal Fermi gas form for $\Delta \mu_{3}(T, x)$, equation (9), in which case one has

$$
\Delta \mu_{4}(T, x)=-\frac{2}{3} x \frac{\Delta U_{\text {idea } 1}\left(T, V_{3}, N_{4}\right)}{N_{3}},
$$

where $\Delta U_{i d e a l}$ is the increase in internal energy relative to $T=0$ for an ideal gas of $N_{3}$ Fermions in a volume determined by $V_{3}$ and $V_{4}$. This quantity may be obtained directly from Tables given by Stoner [13] since one has

$$
\frac{1}{k T_{F}}\left(\frac{\Delta U_{\mathrm{idea}}}{N_{3}}\right)=\frac{E\left(k T / \varepsilon_{0}\right)}{N \varepsilon_{0}}-0.60000,
$$

where Stoner's notation is used for convenience on the right side $\left(J \rightarrow E ; k T_{F} \rightarrow \varepsilon_{0} ; V_{3} \rightarrow V\right)$ and where $T_{F}$ is obtained from equation (7). Setting $x_{0}=0.0635$ and solving equation (37) as indicated we find the variation of $x$ with $T$ shown on Fig. 5. At high temperature we find $x T=4.7 \times$ $10^{-3}{ }^{\circ} \mathrm{K}$. This may be conpared with equation (19), where constancy of $\mu_{4}$ would imply constancy of $P_{\text {ext }}$ and hence of the product $x T$. At low temperatures we find

$$
\Delta x=x-x_{0}=-3.3 T^{2} \mathrm{~K}^{0-2}, \quad(T \rightarrow 0) .
$$

The cooling power of $\mathrm{He}^{3}$ in the dilute phase may now be calculated. It will be expressed in terms of an effective enthalpy. The thermodynamic analysis is facilitated by considering the diagram shown in Fig. 9. In order to discuss a fixed number of $\mathrm{He}^{3}$ atoms, imagine some dilute solution at $T, x(T)$ [or with some distribution of $T, x(T)$ which does not change as other changes are made] and an incremental amount of solution containing $n_{3}$ atoms at temperature $T_{1}$ and concentration $x\left(T_{1}\right)$ which are separated from pure $\mathrm{He}^{4}$ by means of the set of superleak pistons shown. These enable the condition $\mu_{4}=$ constant to be fulfilled. In the process considered, the lower piston moves up until it reaches the final position shown while the upper piston moves up until $n_{3}$ atoms at temperature $T$ and concentration $x(T)$ are in the upper cylinder. Heat $Q$ is added to maintain the temperature constant at any given position. Details of the analysis are given in Ref. 11. The heat $Q$ may be expressed as a difference in enthalpies

$$
Q=n_{3}\left[h(T, x(T))-h\left(T_{1}, x\left(T_{1}\right)\right] .\right.
$$

where $h$ is an enthalpy function for the dilute solution given by the equation

$$
h[T, x(T)]=\left[\mu_{3_{d}}(0, x(T))-\mu_{3_{d}}\left(0, x_{0}\right)\right]+\frac{5}{3} \frac{\Delta U_{\text {idea } 1}(T, x(T))}{N_{3}}
$$


In equation (44) the quantity $\Delta ! I_{\text {ideal }} / N_{3}$ is given by equation (41), which may be evaluated directly from Stoner's Tables [13]. Numerical evaluation of the first term in equation (44) is performed using equation (10a) with $x_{0}=0.0535$ and $x(T)$ given by the solution of equation (37) which is plotted on Fig. 5.

At low teinoeratures $\Delta x$ varies quadratically with temperature, equation (42), so the first terin on the right side of equation (44) varies as $T^{2}$. It has a negative sign since the dominant

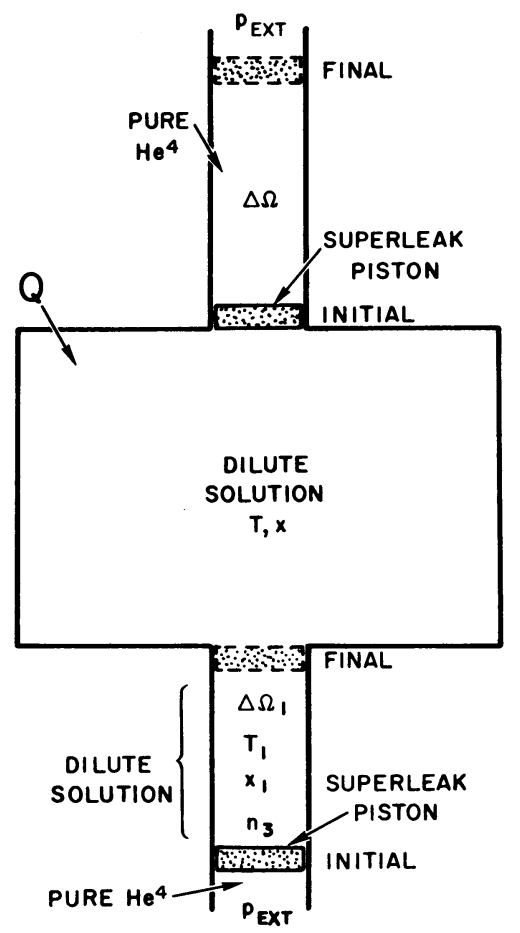

FIGURE 9

Diagram illustrating the analysis of the dilute solution side of a heat exchanger in the continuously operating dilution refrigerator.

contributor to $\mu_{3}(0, x)$ near $x=x_{0}$ is the Fermi energy, which decreases as $T$ increases or $x$ decreases. The second term on the right side of equation (44) is approximated at low temperatures by $\frac{5 \pi^{2}}{12} \frac{k T^{2}}{T_{F}}$. The approximate numerical equation for $h$ at low $T$ is given by

$$
h(T)=6.5 k T^{2} \mathrm{~K}^{\mathrm{O}^{-1}} .
$$

The enthalpy function $h$ over an extended temperature range is plotted on Fig. 7. As $x$ decreases 
the Fermi temperature $T_{F}$ decreases and therefore leads to a higher average kinetic energy for the same $T$ than that for a solution having the limiting low $T$ value of $T_{F}$. This effect is responsible for the upward curvature of $h(T)$ at intermediate temperatures.

We wish to emphasize that the numerical results given in Figs. 5 and 7 for $x(T)$ and $h(T)$ are only approximate since they are based on the approximate expression equation (10a) for $\mu_{3_{d}}(0, x)$. However, these results are very useful for semiquantitative design purposes.

\section{Heat Transfer}

In a continuously operating dilution refrigerator it is necessary for the incoming strean of concentrated $\mathrm{He}^{3}$ to be cooled by transferring heat to the $\mathrm{He}^{3}$ in dilute solution which is flowing away from the mixing chamber. Inspection of equation (22) shows that the limiting low temperature achieved by the helium in the mixing chamber is determined, aside from intrinsic dynamic effects not discussed in the thermodynamic analysis, by the temperature $T_{n}$ at which copcentrated $\mathrm{He}^{3}$ enters the mixing chamber. Hence $T_{n}$ must be reduced as much as possible.

In low-temperature experiments involving helium in which heat must be conducted across a solid-helium interface the so-called thermal-boundary resistance, or Kapitza resistance, is of great importance. A boundary resistivity is defined as the ratio of the temperature jump across the interface separating solid and helium to the heat flux across the interface. The resultant thermal resistance varies inversely as the area of contact. At low $T$ the thermal boundary resistivity may be very large, so to reduce the thermal resistance large contact areas are desirable. For this reason we have used as a heat-exchange medium sintered-copper powder embedded in a copper block. In this case the complete thermal path from the concentrated $\mathrm{He}^{3}$ to the $\mathrm{He}^{3}$ in dilute solution consists of the following thermal resistances in series: intrinsic concentrated $\mathrm{He}^{3}$ resistance, boundary resistance from pure $\mathrm{He}^{3}$ to copper, resistance of the copper block and the sintered copper, thermal resistance from copper to $\mathrm{He}^{3}$ in dilute solution. The intrinsic $\mathrm{He}^{3}$ resistance is known, Fig. 3, and does not represent a problem. Extensive measurements of the thermal boundary resistance between either pure $\mathrm{He}^{3}$ or pure $\mathrm{He}^{4}$ and copper have been made by Anderson, Connolly and Wheatley [41]. They find that below $100 \mathrm{~m}{ }^{\circ} \mathrm{K}$ the thermal boundary resistivity between low pressure $\mathrm{He}^{3}$ and electropolished copper is given by

$$
R_{\mathrm{He}}{ }^{3}-\mathrm{Cu}=\frac{2 \times 10^{-5}}{T^{3}} \mathrm{sec} \mathrm{cm}^{2} \mathrm{~K}^{\circ} / \mathrm{erg} \text {, }
$$

while the boundary resistivity for pure $\mathrm{He}^{4}$ and electropolished copper is less than that given by (46) but increasing relatively as $T$ decreases. The results are summarized on Fig. 10. The thermal resistance of the copper block and sintered copper may be estimated rather well by measuring an appropriate electrical resistance and using the Wiedemann-Franz law to relate the residual electrical resistance to the thermal resistance. This resistance is not a serious problem.

The thermal resistance between copper and dilute solutions has never been measured, excepting some new measurements presented here. It has some rather interesting features. According to the theory of Khalatnikov [42] heat is transferred across the interface by the radiation of sound. That is why the temperature dependence in equation (46) is $T^{-3}$. Parenthetically, only in the case of $\mathrm{He}^{3}$ at low temperatures has the $T^{-3}$ dependence been observed experimentally, the value of the coefficient being about five times less than theory [43]. The temperature jump is associated with the large acoustical impedance mismatch between the copper and helium. Now in 
the case of dilute solutions if the energy were to be transmitted directly from the walls into collective density modes of the $\mathrm{He}^{3}$ quasiparticles (which have all the heat capacity), the acoustic mismatch would be so great that the thermal resistivity would be orders of magnitude greater than given by equation (45). Thus, at least at high temperatures, it is most probable that energy is first transferred into $\mathrm{He}^{4}$ phonons which then give up energy to the $\mathrm{He}^{3}$ quasiparticles via the $\mathrm{He}^{3}$ quasinarticle-He ${ }^{4}$ phonon interaction. The latter has recently been treated by Baym and Ebner [34], who find that at sufficiently low temperatures the mean-free patin (nainly due to absorption of a $\mathrm{He}^{3}$ phonon by the mechanism responsible for ultrasonic attenuation) becomes very long indeed. In this case $\mathrm{He}^{4}$ phonons emitted by the wall are simply

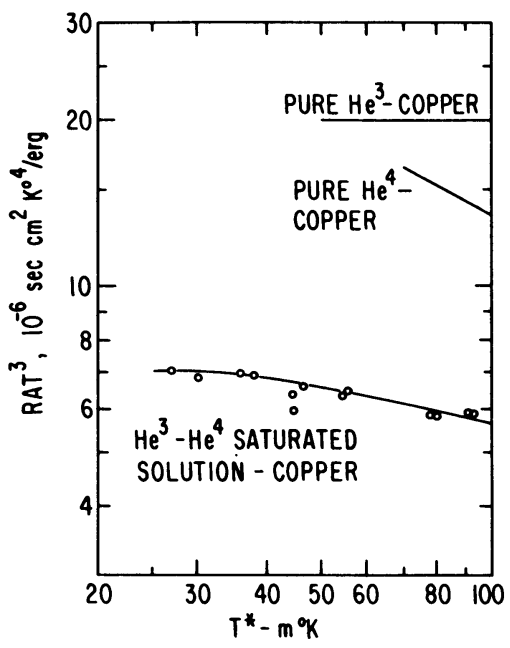

FIGURE 10

Thermal boundary resistance between machined copper and a nearly saturated solution of $\mathrm{He}^{3}$ in $\mathrm{He}^{4}$. The measured thermal boundary resistance $R$ is multiplied by the area $A$ to reduce it to a specific quantity and by $T^{3}$ to remove most of the temperature dependence. Results from Ref. 41 for the thermal boundary resistance between electropolished copper and both pure $\mathrm{He}^{4}$ and pure $\mathrm{He}^{3}$ are shown for comparison.

reabsorbed, for the most part, without transferring energy to the $\mathrm{He}^{3}$. The temperature of the $\mathrm{He}^{4}$ phonon system will rise substantially above that of the $\mathrm{He}^{3}$ system, which is maintained in thermal equilibrium by the frequent interquasiparticle collisions and the high thermal conductivity, Fig. 3. This phenomenon may be described as the $\mathrm{He}^{3}$ quasiparticle-He ${ }^{4}$ phonon thermal resistance, which should decrease as the volume of dilute solution in an exchanger increases. At low temperatures this resistance may become so large that heat is transferred directly into the $\mathrm{He}^{3}$ system, possibly not in collective modes but directly into quasiparticle modes.

In order to estimate the $\mathrm{He}^{3}$ quasiparticle-He 4 phonon resistance, imagine that there are two sets of phonons, one at temperature $T_{1}$ in equilibrium with the walls and a second at temperature $T_{2}$ in equilibrium with the $\mathrm{He}^{3}$. In the absence of any driving heat flow, any initial internal 
energy difference $\Delta U_{0}$ between the two sets of phonons will be assumed to relax by the formula

$$
\Delta U(t)=\Delta U_{0} e^{-t / \tau}
$$

where $t$ is time and $\tau$ is a relaxation time suitably averaged over frequency. At any instant the rate of transfer of energy between the two sets of phonons is

$$
\dot{Q}=-\frac{d \Delta U}{d t}=\frac{1}{\tau} \Delta I(t) .
$$

Now if a constant heat flow $\dot{Q}$ between the two sets of phonons is present, then $\Delta U$ will stabilize with $T_{2} \neq T_{1}$ and

$$
\dot{Q}=\frac{1}{\tau}\left[U\left(T_{1}\right)-U\left(T_{2}\right)\right]
$$

The quantity $U$ is the internal energy of $\mathrm{He}^{4}$ phonons with sound velocity $c_{1}$ in volume $\Omega$ at temperature $T$ given by

$$
U=\frac{\pi^{2}}{30} \frac{\Omega}{\hbar^{3} c_{1}^{2}}(k T)^{4}
$$

This leads to a $\mathrm{He}^{3}$-phonon resistance from equation (49) given by

$$
R_{\text {phonon-He }}{ }^{3}=\frac{\Delta T}{\dot{Q}}=\frac{15}{2 \pi^{2}} \frac{\hbar^{3} c_{1}{ }^{3} \mathrm{~T}}{\Omega k^{4} T^{3}} .
$$

Numerical evaluation of this equation gives

$$
R_{\text {phonon }-\mathrm{He}^{3}}=\frac{3.3 \times 10^{-5} \mathrm{~T}}{\Omega T^{3}} \frac{\mathrm{cm}^{4} \mathrm{~K}^{0^{4}}}{\mathrm{erg}} .
$$

This shows the expected inverse dependence on helium volume in the exchanger. The time $T$ may be estimated to be $\tau=\lambda / c_{1}$ from values given by Baym and Ebner [34] for the mean free path $\lambda$ valid for thermal conduction by $\mathrm{He}^{4}$ phonons. At low $T$ they find $\mathrm{T}-\mathrm{T}-2$ (the temperature dependence of the viscosity of the $\mathrm{He}^{3}$-quasiparticle gas), so that this thermal resistance varies as $T^{-5}$ at low $T$.

The total thermal resistance between copper and $\mathrm{He}^{3}$ in dilute solution is thus the sum of two resistances, one inversely proportional to the area of contact and a second to the volume of the solution. It would be very interesting indeed to conduct a series of experiments in which the ratio of area to volume was changed systematically so that the two resistances could be separated. To date we have done only one such experiment, but its results are quite interesting.

Our standard method [41] for measuring thermal boundary resistance, which is to measure the temperature difference between two copper bodies separated by helium when one is heated at a steady rate and the other maintained at constant temperature, will not work in the present case. That is because heat would flow between the two bodies whether the $\mathrm{He}^{3}$ was present or not. We have therefore developed the simple continuous flow method illustrated schematically in Fig. 11. The mixing chamber of a continuously operating dilution refrigerator contains powdered CMN (cerium magnesium nitrate) for temperature measurement and a heater located above it to allow the temperature to be changed. $\mathrm{He}^{3}$ in the dilute phase is caused to pass out of the bottom of 
the mixing chamber. Its temperature is measured by the $\mathrm{CMN}$. The $\mathrm{He}^{3}$ passes through a hollow copper chambér $0.635 \mathrm{~cm}$ in diameter and $1.316 \mathrm{~cm}$ long provided with a resistance thermometer and a heater. The boundary resistance is high enough and the $\mathrm{He}^{3}$ circulation rate large enough

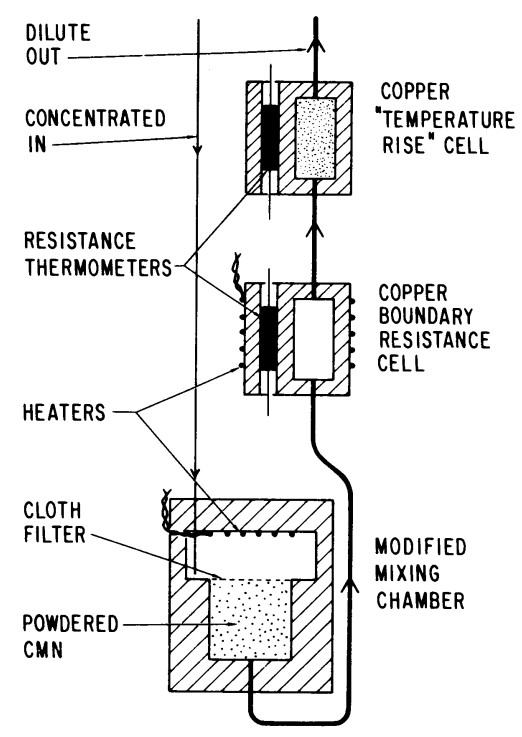

FIGURE 11

Schematic diagram of apparatus used to measure the thermal boundary resistance between a nearly saturated dilute solution of $\mathrm{He}^{3}$ in $\mathrm{He}^{4}$ and copper. This apparatus is placed below the heat exchangers of a continuously operating dilution refrigerator. The phase boundary between $\mathrm{He}^{3}$ and the dilute solution is above the C!N in the modified mixing chamber. Resistance thermometers are Speer carbon resistors made from 1002 Mix and of value $100 \Omega, 1 / 4 \mathrm{~W}$.

that the $\mathrm{He}^{3}$ temperature rise is small compared with that of the copper walls. The temperature rise of the $\mathrm{He}^{3}$ is measured by next allowing the $\mathrm{He}^{3}$ to pass through a copper block containing sintered copper powder and provided with a resistance thermometer. The resistance thermometers are calibrated by assuming that when no heat is added to the hollow copper chamber they have the same temperature as the CMN. The thermal resistance was then obtained from the equation

$$
\frac{\Delta T}{\dot{Q}}=\frac{\left(T_{\mathrm{A}}-T_{\mathrm{CMN}}\right)-1 / 2\left(T_{\mathrm{B}}-T_{\mathrm{CMN}}\right)}{\dot{Q}}
$$

where $T_{A}$ is the temperature of the hollow copper and $T_{B}$ that of the sintered copper. The second term was typically less than $10 \%$ of the first for a molar flow rate of about $2 \times 10^{-5} \mathrm{moles} / \mathrm{sec}$. The resistance was assumed to be valid for the temperature $T=1 / 2\left[T_{A}+1 / 2\left(T_{\mathrm{CMN}}+T_{B}\right]\right.$. The results, multiplied by the inside surface area to reduce them to a resistivity, are shown in Fig. 10. 
They are also multiplied by $T^{3}$ to remove most of the temperature dependence. Plotting the results logarithmically over the range $27 \mathrm{~m}^{\circ} \mathrm{K}-92 \mathrm{~m}^{\circ} \mathrm{K}$, we find $R \propto T^{-3.2}$. These results actually correspond to $\mathrm{He}^{3}$ in a saturated (or nearly saturated) solution with He${ }^{4}$. Thus $x$ is not constant but increases from $6.4 \%$ to about $6.9 \%$ as $T$ increases. The departure of $R$ from the $T^{-3}$ law may be attributed partly to this cause or partly to the $\mathrm{He}^{3}$-phonon resistance discussed above. In view of the history of thermal boundary resistance measurements, it is remarkable that anything as close to the $T^{-3}$ law was observed. The magnitude of the resistivity is about a third of that for pure $\mathrm{He}^{3}$-electropolished copper in the same temperature range, but the present sample was not electropolished and may have a higher effective area than the geometrically calculated area.

According to the calculations of Baym and Ebner [34] for a 5\% solution, the mean-free path for collision of a $\mathrm{He}^{4}$ thermal phonon with a $\mathrm{He}^{3}$ quasiparticle starts to get larger than the diameter of our hollow copper chamber for $T<30 \mathrm{~m}^{\circ} \mathrm{K}$, so it seems reasonable that the ie ${ }^{3}$-phonon resistance would not play a major role in the experiments presented. However, in an actual heat exchanger it could be a problem if the helium volume were too small and the temperature too low. This is a quantitative matter which can be settled by the following considerations.

For a powder of spheres of diameter $d$ the ratio of area to volume is $6 d^{-1}$. For powders used in our experiments a reasonable value for $d$ is $40 \mu \mathrm{m}$, in which case the surface to volume ratio would be $1.5 \times 10^{3} \mathrm{~cm}^{-1}$. The thermal boundary resistance $?_{K}$ may then be expressed in terms of the volume of copper by the conservative estimate

$$
R_{K}=\frac{10^{-8}}{\Omega_{\mathrm{cu} T^{3}}} \operatorname{sec~} \mathrm{K}^{4} \mathrm{~cm}^{3} / \mathrm{erg} \text {, }
$$

where $\Omega_{\mathrm{C}}$ is the volume of copper. The total resistance, using equation (52), is then

$$
R_{\mathrm{tota} 1}=\frac{10^{-8}}{\Omega_{\mathrm{Cu}} T^{3}} \frac{\mathrm{sec} \mathrm{cm}^{3} \mathrm{~K}^{4}}{\mathrm{erg}}+3.3 \times 10^{-5} \frac{\mathrm{T}}{\Omega_{\mathrm{He}} T^{3}} \frac{\mathrm{cm}^{3} \mathrm{~K}^{4}}{\mathrm{erg}}
$$

These two terms may be estimated using for $\tau$ values of the mean-free path computed by Baym and Ebner. [34] for a 5\% concentration. The results are shown in Table 1. For most of the sintered powders which we use the volume of copper and the free volume for helium are comparable. Hence the $\mathrm{He}^{3}$-phonon resistance should not be a problem if the exchanger temperature is above 15 to $20 \mathrm{~m}^{\circ} \mathrm{K}$.

For temperatures higher than the above figure and for the above $40 \mu \mathrm{m}$ powder we can now estimate the necessary size of an exchanger. To simplify the calculations, which are in any case very approximate in view of our imperfect knowledge of the heat exchanger area, we will assume the thermal resistance for both pure $\mathrm{He}^{3}$ and dilute solutions to be given by equation (54). $\mathrm{He}^{3}$ is circulated through the exchanger at a rate $\dot{n}_{3}$. For adequate heat exchange we require that if $t$ is the time $\mathrm{He}^{3}$ is in the exchanger and $\tau$ is the thermal relaxation time for $\mathrm{He}^{3}$ to come in equilibrium with the copper walls, assumed to be at a steady temperature, then $(\tau / t) \ll 1$. Now if $n_{3}$ is the number of $\mathrm{He}^{3}$ atoms in the exchanger then we have $t=n_{3} / \dot{n}_{3}$. We have $\tau=R_{K} C$, where $R_{K}$ is the thermal resistance, assumed given by equation (54), and $C=n_{3} c$, where $c$ is the effective heat capacity per $\mathrm{He}^{3}$ quasiparticle. For pure $\mathrm{He}^{3}$ at low temperatures, $c=k \Gamma T$, where $\Gamma=3.0 \mathrm{~K}^{0^{-1}}$. For the dilute solution at low temperatures $c=d h / d T=k \gamma T$, equation (45) where $\gamma=13 \mathrm{~K}^{-1}$. Thus one has

$$
\frac{T}{t}=\frac{\dot{n}_{3}}{T^{2}}\left(R_{K} T^{3}\right)\left(\frac{c}{T}\right) .
$$




\section{TABLE 1}

Comparison of the product of the thermal boundary resistance $R_{K}$ and the volume of copper with the product of the $\mathrm{He}^{3}$-phonon resistance $R_{\mathrm{He}^{3}}$-phonon and the volume of helium, estimated using the Baym-Ebner theory [34]. The quantity $l_{\text {eff }} 5 \%$ is taken from Table 1, Ref. 34; $T$ is computed by $T=l_{\text {eff }}{ }_{5 \%} / c_{1}$ and used in equation (52) to evaluate $R_{\mathrm{He}^{3} \text {-phonon }}$

\begin{tabular}{|c|c|c|c|c|}
\hline $\begin{array}{l}r \\
\left(\mathrm{~m}^{\circ} \mathrm{K}\right)\end{array}$ & $\begin{array}{l}l_{\text {ef }} \mathbf{f}_{5 \%} \\
(\mathrm{~cm})\end{array}$ & $\begin{array}{c}T \\
\left(10^{-6} \mathrm{sec}\right)\end{array}$ & $\begin{array}{l}\Omega_{\mathrm{He}} R_{\mathrm{He}}^{3}-\mathrm{phonon} \\
\left(\mathrm{cm}^{3} \text { sec } \mathrm{m}^{\circ} \mathrm{K} / \mathrm{erg}\right)\end{array}$ & $\begin{array}{c}{ }^{\Omega_{\mathrm{Cu}}} R_{K} \\
\left(\mathrm{~cm}^{3} \text { sec } \mathrm{m}^{\circ} \mathrm{K} / \mathrm{erg}\right.\end{array}$ \\
\hline 10 & 5.2 & 220 & 7.0 & 10 \\
\hline 15 & 2.2 & 92 & 0.9 & 3 \\
\hline 20 & 1.14 & 48 & 0.2 & 1 \\
\hline 30 & 0.43 & 18.2 & 0.02 & 0.4 \\
\hline 40 & 0.21 & 8.8 & 0.005 & 0.16 \\
\hline
\end{tabular}

If we use the same value of ${ }_{1} K T^{3}$ for both the concentrated $\mathrm{He}^{4}$ and the dilute solution then, since $\gamma / \Gamma=4.3$, the dilute exchanger will require about four times as much volume of copper as the concentrated. For $n_{3} / A_{0}=3 \times 10^{-5} \mathrm{moles} / \mathrm{sec}$ and $\Omega_{\mathrm{C}_{u}}=1 \mathrm{~cm}^{3}$ we find $(\tau / t)_{c}=\left(9 \mathrm{~m}^{\circ} \mathrm{K} / T\right)^{2}$ for the concentrated phase and $(\tau / t)_{d}=\left(18 \mathrm{~m}^{\circ} \mathrm{K} / T\right)^{2}$. Here $A_{0}$ is Avogadro's number.

\section{Circulating $\mathrm{He}^{3}$}

Dilution refrigerators which operate by virtue of an efflux of $\mathrm{He}^{3}$ from the mixing chamber require a gradient in the real osnotic pressure to force the $\mathrm{He}^{3}$ against viscous resistance through the superfluid. Continuously operating devices require moreover that the $\mathrm{He}^{3}$ be concentrated before being recirculated. As suggested by London et al. [5] an effective method of concentrating the $\mathrm{He}^{3}$ is to distill it from the dilute superfluid solution, even when the $\mathrm{He}^{3}$ concentration is less than $1 \%$. In the original suggestion the $\mathrm{He}^{3}$ vapors from the still were to be recondensed at low temperatures $\left(\sim 0.3\right.$ to $\left.0.4^{\circ} \mathrm{K}\right)$, but H.E. Hall [7] pointed out that the refrigerator would also work well if $\mathrm{He}^{3}$ were simply evaporated from a still, compressed to a suitable pressure at room temperature, and then recondensed and recirculated. Hall's suggestion has the advantage of technical simplicity.

It is important that $\mathrm{He}^{3}$ and not $\mathrm{He}^{4}$ be circulated. This may be understood by referring to Fig. 2. Suppose for the sake of definiteness that the circulated gas contains only $80 \% \mathrm{He}^{3}$. After this gas is condensed it is normal fluid. Its temperature is reduced by contact with the still and by other heat exchange with the dilute solution effluent. Below about $0.7^{\circ} \mathrm{K}$ phase separation starts. In order to cool the two-phase mixture further it is necessary not only to remove energy from the different phases but also to remove the "heat of separation". Just as the process of dilution of $\mathrm{He}^{3}$ with $\mathrm{He}^{4}$ at constant $T$ results in absorption of heat, separation of $\mathrm{He}^{3}$ from $\mathrm{He}^{4}$ results in the evolution of heat. The effect is greatest where the slope of the phase-separation curve is least. Below $0.1^{\circ} \mathrm{K}$ the effect is entirely negligible, but the heat of separation can constitute a substantial load for the first heat exchanger below the still. For $\mathrm{He}^{3}$ concentrations in the circulated vapor less than about $67 \%$ the condensed fluid is superfluid. 
We have not considered in detail what might happen in this case, but some peculiar phenomena associated with the superfluid might occur.

The above remarks on heat of separation also apply to the single-cycle dilution refrigerator when it is used to produce low temperatures. Its mixing chamber must be precooled to a temperature sufficiently low that the heat of separation does not constitute a serious load on the available refrigeration.

In designing a still it is necessary to minimize the $\mathrm{He}^{4}$ circulated while providing an adequate $\mathrm{He}^{3}$ vapor pressure to drive the desired flow through the external pumping system. The thermodynamic equilibrium vapor pressure of $\mathrm{He}^{3}$ in the still may be calculated by equating the partial chemical potentials for $\mathrm{He}^{3}$ in the vapor and in the superfluid. For the vapor, we use the ideal gas expression

$$
\mu_{3 v}=k T\left[\ln \frac{P_{3}}{k T}+\ln 1 / 2\left(\frac{2 \pi \kappa^{2}}{m_{3} k T}\right)^{3 / 2}\right]
$$

while for $\mathrm{He}^{3}$ in dilute solutions at high temperature and low concentration we use the ideal Boltzmann gas expression

$$
\mu_{3}=k T\left[\ln \frac{x}{\omega_{0,4}}+\ln 1 / 2\left(\frac{2 \pi \hbar^{2}}{m^{*} k_{i}^{i}}\right)^{3 / 2}\right]-E_{0_{3}},
$$

where $\omega_{0,4}$ is the volume per atom in pure $\mathrm{He}^{4}$ and $E_{0_{3}}$ is the binding energy. The He ${ }^{3}$ vapor pressure $P_{3}$ is then

$$
P_{3}=\frac{k T x}{\omega_{0,4}}\left(\frac{m_{3}}{m^{*}}\right)^{3 / 2} \exp \left(-E_{0_{3}} / k T\right) .
$$

In this equation at low $\mathrm{He}^{3}$ flow rates through the superfluid phase and at low mixing chamber temperature $T_{x}$ is a constant (Fig. 5) equal to $4.7 \times 10^{-3}{ }^{\circ} \mathrm{K}$, according to our calculation. For numerical evaluation of this equation use $E_{0_{3}} / k=2.7^{\circ} \mathrm{K}, \mathrm{m}^{*} / \mathrm{m}_{3}=2.5$, and $A_{0} \omega_{0,4}=27.5 \mathrm{~cm}^{3} / \mathrm{mole}^{2}$ $\left(A_{0}\right.$ is Avogadro's number) to find

$$
P_{3}=2.9 \exp \left(-2.7^{\circ} \mathrm{K} / T\right) \mathrm{mm}-\mathrm{Hg} .
$$

For any nonzero $\mathrm{He}^{3}$ flow rate, $x$ at the still falls below the value computed from $T x=4.7 \mathrm{x}$ $10^{-3}{ }^{\circ} \mathrm{K}$ as a result of viscous flow impedance requiring the development of an osmotic pressure difference to drive the $\mathrm{He}^{3}$ flow. Numerical evaluation of equation (18) at $T=0$ using equation (10a) leads to a maximum possible osmotic pressure difference (corresponding to $x=0$ at the stili) of $10.6 \mathrm{~mm}-\mathrm{Hg}$. This is not a large pressure in relation to the large $\mathrm{He}^{3}$ molar volume in the dilute phase and the high viscosity at low temperatures of Fermi fluids. Hence great care must be used in keeping the flow impedance for the $\mathrm{He}^{3}$ in dilute solution as low as possible. Otherwise, $x$ drops in the still. The temperature of the still at a given vaporization rate the rises, and the fractional amount of $\mathrm{He}^{4}$ circulated rises with attendant harmful effects as indicated above. Using the vapor pressure of pure $\mathrm{He}^{4}$ to approximate that of the $\mathrm{He}^{4}$ in the cillute solution, we find the results shown in Table 2.

The ratio of $\mathrm{He}^{4}$ to $\mathrm{He}^{3}$ in the circulated gas will always be greater than that indicated in 
Table 2 as a result of $\mathrm{He}^{4}$ film flow. About the lowest film flow rates are observed for glass substrates, where a typical flow rate per unit length of film perpendicular to the flow is $8 \times 10^{-5} \mathrm{~cm}^{3} / \mathrm{sec} / \mathrm{cm}$ [44]. Metal substrates sometimes lead to flow rates [44] several times higher than this figure, though a high polish tends to bring flow rates over metals closer to that over glass. Below $1^{\circ} \mathrm{K}$ the film flow rate increases somewhat [45], though the effect is not large.

TABLE 2

Calculated partial vapor pressure of $\mathrm{He}^{3}$ in the still, and thermodynamic equilibrium ratio of the partial vapor pressure of $\mathrm{He}^{4}$ to that of $\mathrm{He}^{3}$ for various still temperatures.

\begin{tabular}{ccc}
\hline \hline$T_{\text {still }}$ & $P_{3}$ & $P_{4} / P_{3}$ \\
$\left({ }_{\mathrm{K}}\right)$ & (microns-Hg) & \\
\hline 0.50 & 14 & $1.1 \times 10^{-3}$ \\
0.55 & 23 & $3.3 \times 10^{-3}$ \\
0.60 & 34 & $8.2 \times 10^{-3}$ \\
0.65 & 48 & $1.8 \times 10^{-2}$ \\
0.70 & 64 & $3.5 \times 10^{-2}$ \\
0.75 & 83 & $6.4 \times 10^{-2}$ \\
0.80 & 103 & $1.1 \times 10^{-1}$ \\
0.85 & 126 & $1.8 \times 10^{-1}$ \\
\hline
\end{tabular}

For any given still and pumping configuration the ratio of $\mathrm{He}^{3}$ to $\mathrm{He}^{4}$ in the circulated vapor will exhibit a maximum as a function of still temperature. At low $T$ the nearly temperature independent $\mathrm{He}^{4}$ film flow rate will dominate since the $\mathrm{He}^{3}$ flow rate, dependent on the $\mathrm{He}^{3}$ vapor pressure, will be quite small. At high enough $T$, the equilibrium $\mathrm{He}^{3}$ and $\mathrm{He}^{4}$ vapor pressures are high enough to make the film flow contribution of $\mathrm{He}^{4}$ small, but the relative amount of $\mathrm{He}^{4}$ increases with increasing $T$ for thermodynamical reasons. The $\mathrm{He}^{4} \mathrm{film}$ flow rate may be reduced by pumping the still through a film flow limiting orifice. The optimum size of this orifice is determined by the requirement that, for the normal molar flow rate desired for the refrigerator, the pressure drop across the orifice be an appreciable fraction of the $\mathrm{He}^{3}$ vapor pressure. If the orifice is too small, the still temperature will have to be too high to get the desired flow rate, so the $\mathrm{He}^{3} / \mathrm{He}^{4}$ ratio will decrease. If the orifice is too large, the $\mathrm{He}^{4}$ film flow will decrease the $\mathrm{He}^{3} / \mathrm{He}^{4}$ ratio. A detailed experimental study will be presented in a later section.

There is one further effect of circulating $\mathrm{He}^{3}$ which should be mentioned. This is concerned with the effects of viscous heating on the low-temperature parts of the apparatus, where the $\mathrm{H}^{3}$ viscosity may be rather high. For laminar flow, the pressure drop $\Delta P$ required to drive a volume flow $\dot{V}$ of a fluid with viscosity coefficient $\eta$ is

$$
\Delta P=Z \cap V .
$$

where $Z$ is a quantity, depending only on geometry, which we call the impedance factor, or impedance for short. In analogy to $0 \mathrm{hm}$ 's law for electrical circuits, $Z_{\eta}$ might better be labeled the impedance, but $Z$ is the more convenient quantity to deal with experimentally since it is 
independent of gas and temperature (excepting effects of thermal expansion) and may be measured by flow experiments at room and nitrogen temperatures. For a tube of diameter $d$ and length $l$, the impedance factor is

$$
Z=\frac{128 l}{\pi d^{4}}
$$

For two parallel plates $Z=12 l / w t^{3}$, where $w$ is the width of the plates and $t$ is the thickness of the gap. Comparison of this formula with equation (52) shows that $Z$ varies inversely as the cross sectional area and then as the inverse square of the smallest characteristic dimension. We have found that the practical unit of impedance for our work with dilution refrigerators is $10^{8} \mathrm{~cm}^{-3}$.

The viscous heating rate $\dot{Q}_{v}$ is just $\dot{V}_{\Delta}^{p}$, so one has

$$
\dot{Q}_{v}=Z_{\eta} \dot{V}^{2} \text {. }
$$

For pure $\mathrm{He}^{3}$, the volume flow rate is $35.8 \dot{n}_{3} / A_{0} \mathrm{~cm}^{3} / \mathrm{mole}$, where $\dot{n}_{3} / A_{0}$ is the molar flow rate. For dilute solutions at low temperature where $x=0.0635$, the volume flow rate is 433 $\dot{n}_{3} / A_{0} \mathrm{~cm}^{3} / \mathrm{mole}$. The viscous heating $\dot{Q}_{v}$ results in a temperature increase $\Delta T$ of the flowing $\mathrm{He}^{3}$ which may be computed from the effective specific heat per particle,

$$
C_{c}=k \Gamma T, \Gamma=3.0 \mathrm{~K}^{0-1} \text {. }
$$

for concentrated $\mathrm{He}^{3}$; and, setting $C_{\breve{u}}=d h / d T$ (equation 45 ),

$$
C_{d}=k_{\gamma} T, \gamma=13 \mathrm{~K}^{\circ-1} \text {, }
$$

for the dilute solution. Both equations (64) and (65) are valid at low $T$ only. One then has, $\frac{\Delta T}{T} \ll 1$,

$$
\frac{\Delta T}{T}=\frac{\eta T^{2}\left(\dot{V} A_{0} / \dot{n}_{3}\right)^{2}}{\left(A_{0} C / T\right)}\left(\frac{Z_{1_{3}} / A_{0}}{T^{4}}\right)
$$

Using equations (12), (14), (64), (55) and the above figures for the ratio of volume to molar flow rates, we find the following numerical results:

$$
\left(\frac{\Delta T}{T}\right)_{c}=0.07\left(\frac{Z}{10^{8} \mathrm{~cm}^{-3}}\right) \quad\left(\frac{\dot{n}_{3} / A_{0}}{10-5 \mathrm{moles} / \mathrm{sec}}\right) \quad\left(\frac{20 \mathrm{~m}^{\circ} \mathrm{K}}{T}\right)^{4}
$$

for the concentrated phase, and

$$
\left(\frac{\Delta T}{T}\right)_{d}=0.54\left(\frac{Z}{10^{8} \mathrm{~cm}-3}\right)\left(\frac{\dot{n}_{3} / A_{0}}{10^{-5} \mathrm{moles} / \mathrm{sec}}\right)\left(\frac{20 \mathrm{~m}^{\circ} \mathrm{K}}{T}\right)^{4}
$$

for the dilute phase. Inspection of (67) and (68) shows that for identical conditions the viscous heating problem is about eight times greater for the dilute compared with the concentrated phase. Furthermore, $(\Delta T / T)$ increases as $T^{-4}$ so that the viscous heating problem can 
be expected to set in quite rapidly below a certain temperature region. Also, for a given exchanger the dilute phase will be colder than the concentrated phase, accentuating the comparison between (57) and (58). All that one can do is decrease $Z$ for the dilute phase as much as possible, but this may not be simple to do technically while maintaining adequate heat exchange capability.

\section{Intrinsic Factors}

In this section we consider some factors which are effective in limiting the low temperature which can be achieved in a $\mathrm{He}^{3}$ flow-type dilution refrigerator, exclusive of such matters as the residual heat leak and the temperatures of surrounding media. As indicated in the previous section, flowing $\mathrm{He}^{3}$ is attended by a viscous heating proportional to the viscosity coefficient and to the square of the $\mathrm{He}^{3}$ flow rate, equation (63). The resultant temperature rise per unit length, equation (66), is linearly proportional to the $\mathrm{He}^{3}$ flow rate since the capability to absorb heat is proportional to the flow rate. Even taking thermal conduction into account, the viscously induced temperature gradient varies linearly with $\mathrm{He}^{3}$ flow rate. Now in the case of the single-cycle refrigerator, where there is no heat exchange problem, the refrigeration available at a given $T$ is linearly proportional to $\mathrm{He}^{3}$ flow rate. Hence the mixing chamber of such a refrigerator will decrease in temperature until the heat conducted in by virtue of the viscously induced temperature gradient is compensated by the refrigeration available. The resultant temperature will be independent of $\mathrm{He}^{3}$ flow rate.

In what follows, we will assume that the $\mathrm{He}^{3}$ in dilute solution is flowing through a uniform circular tube of diameter $d$. There is actually a velocity variation perpendicular to the axis of the tube, but, aside from its importance in determining the heating by viscosity, we will assume that the temperature depends only on the distance $l$ along the tube. We do not expect this assumption to have a qualitative effect on the results. Consider two points, 1 and 2 , on the tube separated by the distance $\Delta l$ and with temperatures $T_{1}$ and $T_{2}$ such that $T_{2}=T_{1}+\frac{d T}{d l} \Delta l$. The heating rate of the helium in this section is partly from viscosity in abount [equations (62) and (63)]

$$
\frac{128 \Delta l}{\pi \lambda^{4}} \frac{\left(\eta^{2}\right)}{T_{2}}\left(\frac{\omega_{0,4} 2}{x_{0}}\right) \dot{n}_{3}{ }^{2}
$$

and partly from the difference in the heat brought in by conduction at the two end sections in amount

$$
\frac{\pi d^{2}}{4}(\kappa T)\left[-\frac{1}{T^{2}}\left(\frac{d T}{d l}\right)^{2}+\frac{1}{T} \frac{d^{2} T}{d l^{2}}\right] \Delta l .
$$

Since the results are meant to be applied at low temperatures $\left(<10 \mathrm{~m}^{\circ} \mathrm{K}\right)$ we have used the limiting forms for the transport coefficients given in equations (14) and (15). The above two heating rates are balanced by the rate at which the dilute solution absorbs energy, $\dot{n}_{3}\left[h\left(T_{2}\right)-\right.$

$\left.h\left(T_{1}\right)\right]$, equation (43), which at low $T$ is approximated by $\dot{n}_{3} k y T \frac{d T}{d l} \Delta l$, equation (65). The 
heat balance equation is thus

$$
\left.\frac{128}{\pi d^{4}} \frac{\left(\eta^{2}\right)\left(\omega_{0,4} / x_{0}\right)^{2} \dot{n}_{3}^{2}}{T^{2}}+\frac{\pi d^{2}}{4}(\kappa T) \mid-\frac{1}{T^{2}} \frac{d T}{d l}{ }^{2}+\frac{1}{T} \frac{d^{2} T}{d l^{2}}\right\rfloor=\dot{n}_{3} \gamma k T \frac{d T}{d l}
$$

At high enough temperatures the term in $\mathrm{k} T$ is negligible, and equation (69) may be solved immediately to find

$$
\frac{d T}{d l}=\frac{a \dot{n}_{3}}{T^{3}} \quad(\text { high } T)
$$

where

$$
a=\frac{128}{\pi d^{4}} \frac{\left(\eta T^{2}\right)\left(\omega_{0,4} / x_{0}\right)^{2}}{\gamma^{k}}
$$

At lower $T$ one can substitute

$$
\frac{d T}{d l}=\frac{a \dot{n}_{3}}{T^{3}}(1-f)
$$

into equation (59) to find a differential equation for $f$ given by

$$
t \frac{d f}{d t}=\frac{f}{1-f} t^{6}-4(1-f)
$$

where $t=T / T_{0}$, and the characteristic temperature $T_{0}$ is given by

$$
T_{0}=\left[\begin{array}{ll}
\frac{32}{d^{2}} & \frac{\left(\eta T^{2}\right)(\kappa T)\left(\omega_{0,4} / x_{0}\right)^{2}}{k^{2} \gamma^{2}}
\end{array}\right]^{1 / 6} .
$$

Since $T_{0}$ does not involve $\dot{n}_{3}$, neither does $f$, so the temperature gradient equation (71), depends only linearly on $n_{3}$. The resultant limiting temperature of the mixing chamber is, from equations (24) and (71), determined by the equation

$$
\dot{n}_{3} k \beta T^{2}=\frac{\pi d^{2}}{4} \quad \frac{\left(\kappa^{\prime} T^{\prime}\right)}{T} \frac{a \dot{n}_{3}}{T^{3}}(1-f), \quad \beta=10 \mathrm{~K}^{0-1},
$$

where $T$ is the temperature of the mixing chamber and $d T / d l$ is the temperature gradient at the exit to the mixing chamber. Combining terms and using equations (70) and (73) the limiting temperature is given by the solution of the equation

$$
\frac{\gamma}{\beta} \frac{\left[1-f\left(t_{M C}\right)\right]}{t_{M C}{ }^{6}}=1
$$

where $\gamma / \beta=1.3$ according to our estimates. The dependence of $f$ on $t$ has been determined by numerical integration. The results are shown on Fig. 12. Solutions of equation (72) for higher $t$ may be obtained by integration in series. Using this graph equation (74) is solved for $t_{M C}=$ 0.9. From the values for $\eta T^{2}, k T, \omega_{0,4} / x_{0}$, and $\gamma$ suggested earlier, we find the mixing chamber 
temperature in the single-cycle refrigerator to be limited by intrinsic factors to be greater than

$$
\Gamma_{M C_{\text {intrinsic }}}=4\left(\frac{1 \mathrm{~mm}}{d}\right)^{1 / 3} \mathrm{~m}^{\circ} \mathrm{K}
$$

In the single-cycle device of Vilches and Wheatley [10] the "pumping" tube had $a=0.62 \mathrm{~mm}$, thus corresponding to an intrinsically limited temperature of $4.7 \mathrm{~m} / \mathrm{K}$ according to this formula. A

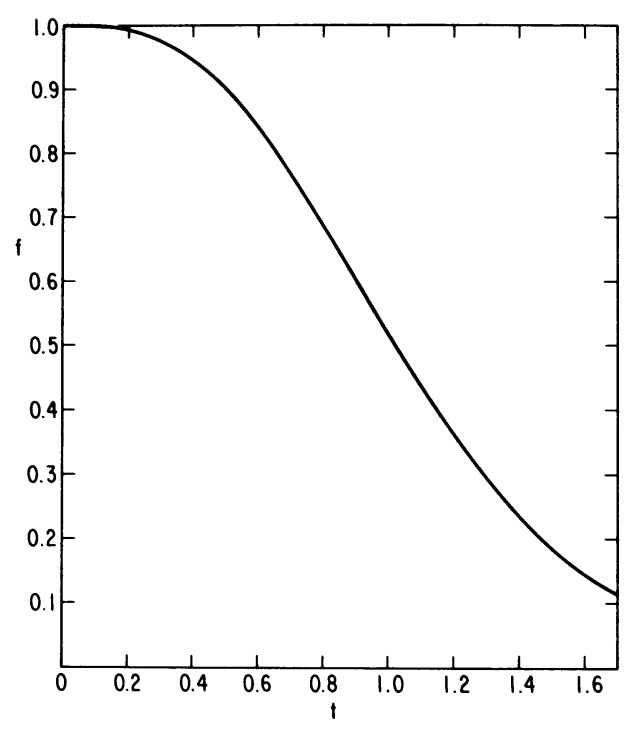

FIGURE 12

Plot of the solution of equation (72) giving $f$, defined in equation (71), as a function of a reduced temperature $t=T / T_{0}$, where $T_{0}$ is given by equation (73).

limiting temperature of $4.5 \mathrm{~m}^{\circ} \mathrm{K}$ was actually observed. Further experimental checks of this analysis will be most interesting, but it seems clear that the $d^{-1 / 3}$ dependence on diameter of $T_{M C_{\text {intrinsic }}}$ will not allow the low temperature to be pushed down substantially by adjustment of the tube diameter. On the other hand the $t^{6}$ temperature dependence in equation (74) suggests that intrinsic factors will not limit the continuous refrigerator until $T$ is just somewhat greater than $5 \mathrm{~m}^{\circ} \mathrm{K}$.

At some sufficiently low temperature the transport coefficients will be limited by boundary scattering so that $\eta$ will become temperature independent and linearly dependent on $d$ while $k$ will be linearly dependent on both $T$ and $d$. In this case the limiting temperature will be determined by an equation qualitatively different from equation (74) with a much weaker temperature dependence. We have not considered this limit in detail. 


\section{General Refrigerator Characteristics}

In Fig. 13 is a schematic diagram of a continuously operating dilution refrigerator based on the ideas discussed in previous sections. One feature of this refrigerator should be mentioned

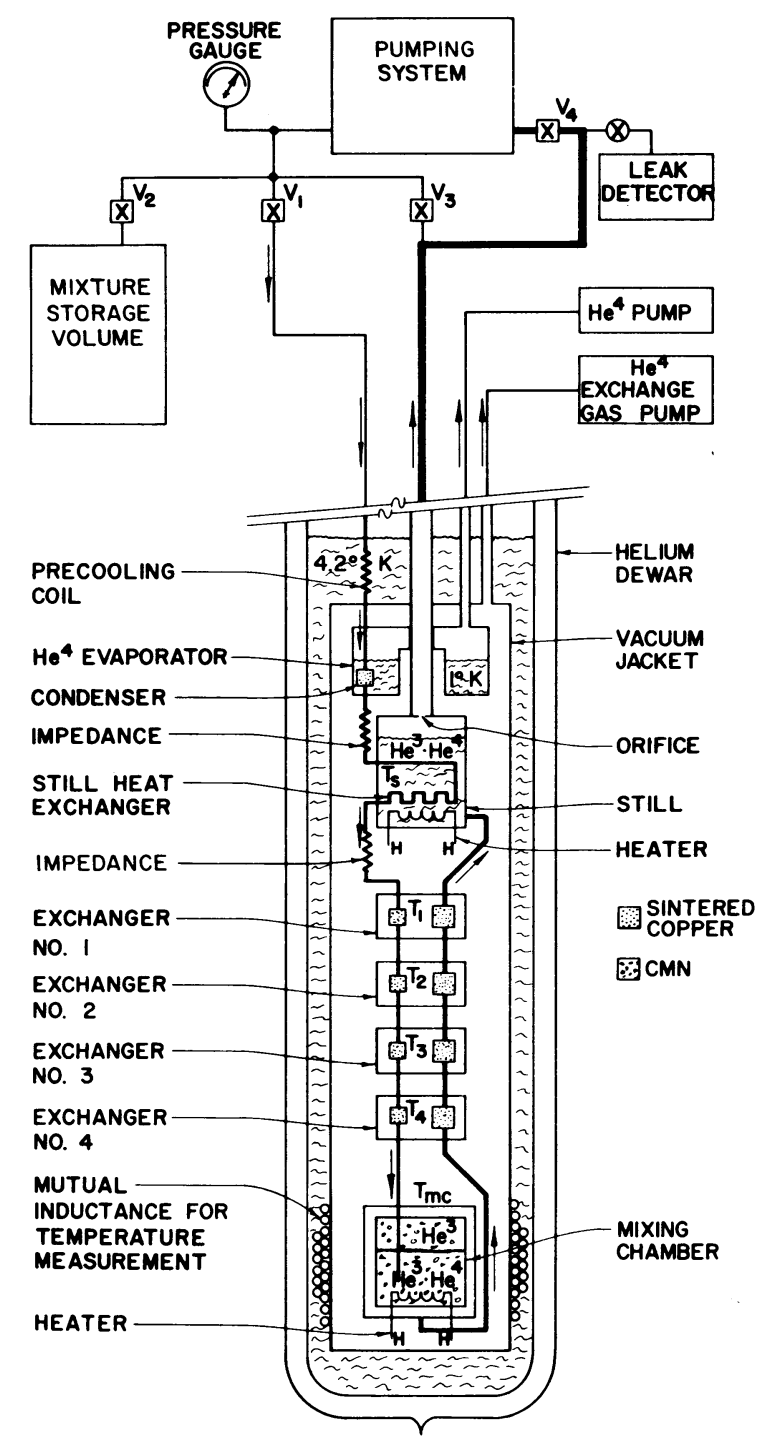

FIGURE 13

Schematic representation of a dilution refrigerator with four heat exchangers.

at the outset. The dilute phase line interconnecting mixing chamber, heat exchangers, and still 
is, as drawn, gravitationally unstable. That is because in the dilute phase the He ${ }^{3}$ concentration decreases as $T$ increases, so the weight density of the fluid increases with height in the arrangement shown. Convective instabilities may then develop which will destroy the thermal isolation of the various stages from one another. However, the possibility of convection is decreased by decreasing tube diameters and by increasing viscosity and thermal conductivity. As the temperature decreases, interconnecting tubes may therefore be increased in diameter. For tube sizes used by us no effects of convection have ever been observed. As indicated on Fig.13, heat exchange occurs in steps. This makes it simple to insure that at least part of each interconnecting tube for dilute solution is gravitationally stable. For a further discussion of this subject, particularly in regard to the problems created by convection in early experimental models, the reader is referred to Ref. 7.

The dilution refrigerator is located within a vacuum jacket surrounded by liquid He 4 boiling at atmospheric pressure. It is mounted below a $\mathrm{He}^{4}$ evaporator which maintains a temperature of about $1^{\circ} \mathrm{K}$. Some technical simplification might be obtained by eliminating this evaporator and pumping the main bath to $1.3^{\circ} \mathrm{K}$ or less. However, refilling the main bath will disturb the operation of the dilution refrigerator. After passing through a precooling coil in the main bath, helium is condensed in a condenser of sintered copper powder. The flow limiting impedance, just after the condenser, has such a high impedance factor that the condenser always operates full of liquid. The input helium is then passed through a tube in the still to exchange heat there and then, in our latest versions, passes a second impedance before reaching the first heat exchanger. This second impedance is designed to force the pressure in the concentrated $\mathrm{He}^{3}$ at the still temperature to be greater than the vapor oressure. Otherwise, vapor will be formed at or before the still and seriously load the first exchanger. Heat exchange then takes place in a number of steps. The number of steps shown in Fig. 13 is four, though we have made measurements with from two to five exchangers. Then the concentrated $\mathrm{He}^{3}$ discharges into the mixing chamber. The discharge tube is shown to terminate part of the way down the mixing chamber. This has the advantage that the flowing concentrated $\mathrm{He}^{3}$ is immediately exposed to superfluid $\mathrm{He}^{4}$, into which it can dissolve readily. If the concentrated $\mathrm{He}^{3}$ is discharged at the top of the mixing chamber, a temperature gradient will exist in the upper phase. With the arrangement shown, it is possible in principle for the superfluid level to rise to the fourth exchanger and spoil the operation of the device, but this will not happen if the impedance of the line on the concentrated side between the last exchanger and the mixing chamber is high enough. The mixing chamber is provided with a powder of cerium magnesium nitrate for temperature measurement by a mutual inductance, method. The $\mathrm{He}^{3}$ then flows out of the mixing chamber, through the heat exchangers, and thence into the still, where it is evaporated. All the heat exchangers, the still, the mixing chamber, and the $\mathrm{He}^{4}$ evaporator are provided with resistance thermometers to monftor the operation of the device. The external pumping system contains a Veeco MS9A Leak Detector modified [46] to enable $\mathrm{He}^{3} / \mathrm{He}^{4}$ ratios to be measured as well as a pressure gauge on the input line to measure the condensation pressure. These instruments enable us to analyze the behaviour of the device and regulate its operation.

An important feature of the device is the number of heat exchangers. On the assumption of ideal heat exchange, no viscous heating, thermal isolation between parts, and no $\mathrm{He}^{4}$ circulated, this number can be estimated given the mixing chamber temperature and the approximate still temperature. We use equation (22), with $Q_{\text {ext }}=0$, and equation (43) with the numerical results given in Fig. 6 [representing equation (27)] and Fig. 7 and low temperature approximations given in equations (24), (28), and (45). Let there be $n$ exchangers. Then conservation of energy in the mixing chamber yields the equation [equation (22)]

$$
\left[u_{3_{c}}\left(T_{n}\right)-u_{3_{c}}\left(T_{M C}\right)\right]=T_{M C} \Delta s\left(T_{M C}\right) \text {. }
$$

where $T_{n}$ is the temperature of the $n$-th exchanger. Conservation of energy at the $n$-th exchanger 
gives

$$
{ }_{u_{3}}\left(T_{n-1}\right)-u_{3_{c}}\left(T_{n}\right)=h\left(T_{n}\right)-h\left(T_{M C}\right) \text {. }
$$

and so on for the other exchangers. Given $T_{M C}$, the right side of equation (75) is given in Fig. 6 (or by equation 27), $u_{3} c\left(T_{M C}\right)$ is given in Fig. 7 (or by equation 28), so $u_{3} c\left(T_{n}\right.$ ) and hence $T_{n}$ can be determined. Given $T_{M C}$ and $T_{n}$, equation (76) and Fig. 7 determine $T_{n-1}$, and so on. For the case $T_{M C}=10 \mathrm{~m}^{\circ} \mathrm{K}$, we find, $T_{n}=28 \mathrm{~m}^{\circ} \mathrm{K}, T_{n-1}=65 \mathrm{~m}^{\circ} \mathrm{K}, T_{n-2}=179 \mathrm{~m}^{\circ} \mathrm{K}, T_{n-3}=$ $730 \mathrm{~m}^{\circ} \mathrm{K}$. The last named temperature is quite reasonable for the still temperature, so only three steps of heat exchange should be necessary under ideal conditions. However, the heat load due to circulated $\mathrm{He}^{4}$ requires at least one more exchanger on the high $T$ end while heat exchange problems and perhaps viscous heating require another heat exchanger on the low $T$ end, so that in practice about five steps have been required to reach $10 \mathrm{~m} \%$.

\section{Dilution Refrigerator Construction}

\section{A. Refrigerator components}

A representation of the actual appearance of the still, heat exchangers, and mixing chamber of our continuously operating dilution refrigerator is given in Fig. 14. The actual form of the tubes interconnecting the various parts is illustrated. The still is shown in cutaway view. Details of the heat exchangers and of one form of mixing chamber are given in Figs. 15 and 16 , respectively. In this paper, we will discuss the characteristics of two entirely different refrigerators built along the general lines indicated. These will be called Refrigerators I and II, II being the latest version and I being itself an improvement over some of our earliest work. It will not be possible to recount all the experiments done with these refrigerators, but at the outset we wish to mention that experiments were performed to test the efficiency of the exchangers and to test for possible temperature gradients along the interconnecting tubes. For example, resistance thermometers were located on the input and output of the still exchanger and on the still itself. In all cases we found the output temperature to be the same as the still temperature. Similarly, resistance thermometers were placed on opposite ends of the cupronickel (70-30) tubes interconnecting the still and the first exchanger, for example, and no change in temperature was detected. That is, the dilute phase $\mathrm{He}^{3}$ for all practical purposes enters the still at the temperature of exchanger No. 1 while the concentrated He ${ }^{3}$ enters exchanger No. 1 at the temperature of the still. Also, five resistance thermometers were placed on or near a single exchanger, one on the body and one at each tube connection and on the cupronickel. Heat exchange was ideal for No. 1, but appeared to worsen at successively lower temperature exchangers. However, our low-temperature resistance thermometry was rather poor and the results have only qualitative significance.

Quantitative information on the two refrigerators is given for compactness in Table 3 . The only detail of Fig. 14 which is rendered too schematically is the "secondary impedance". It is actually a coil of $0.1 \mathrm{~mm}$ bore tubing. We now discuss the individual components of Fig. 14.

a. Still. The still is shown at the top of Fig. 14 in cutaway view. It has two main parts, which we call the still bottom and the still top. This particular design has evolved from a series of earlier models which were more fragile and more difficult to assemble and use. The still bottom is made of brass, for convenience, is roughly $25 \mathrm{~mm}$ in diameter, and is counterbored to receive a screw-attached brass mounting flange as shown. The upper part of the still bottom has a spool-like arrangement to receive the coil of cupronickel tubing which serves as 
heat exchanger. Connections to the still bottom are made by means of four standard copper refrigeration capillary tubes, each $2.1 \mathrm{~mm} 0 . \mathrm{d}$. and $0.79 \mathrm{~mm}$ i.d. All four tubes are hard soldered into the brass in holes which slant upward. The axes of all four holes lie in the same plane, which intersects the flat upper surface of the still bottom in a diameter. The two outboard

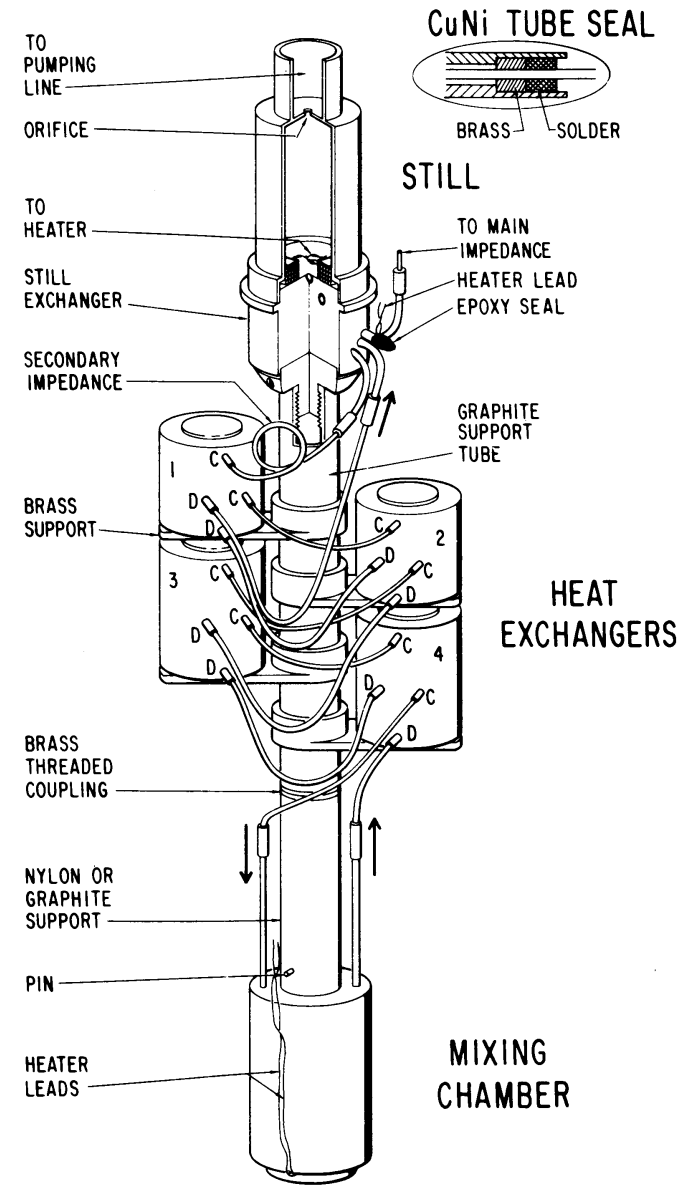

FIGURE 14

\begin{abstract}
Assembly drawing of the still, heat exchangers, and mixing chamber of Refrigerator II. The relative size, relationship, and interconnection of parts is correctly represented. The still is shown in cutaway view to illustrate its construction. The inset at upper right shows the method of sealing the still heat exchanger tube.
\end{abstract}

tubes are used for the exchanger. The two inner tubes break through as shown in the central part. One is used for the dilute phase return line and the other for the still heater leads. 
The copper tubes are soft, yet strong, so they may be bent into any convenient shape without fear of breakage in order to accomnodate the rest of the device. Of the two outboard tubes one breaks through at the inner diameter of the cupronickel exchanger while the other breaks through at the outer dianeter of the exchanger. At these places the brass is carefully beveled and polished to avoid any possible damage to the tube. To assemble the heat exchanger tube into the

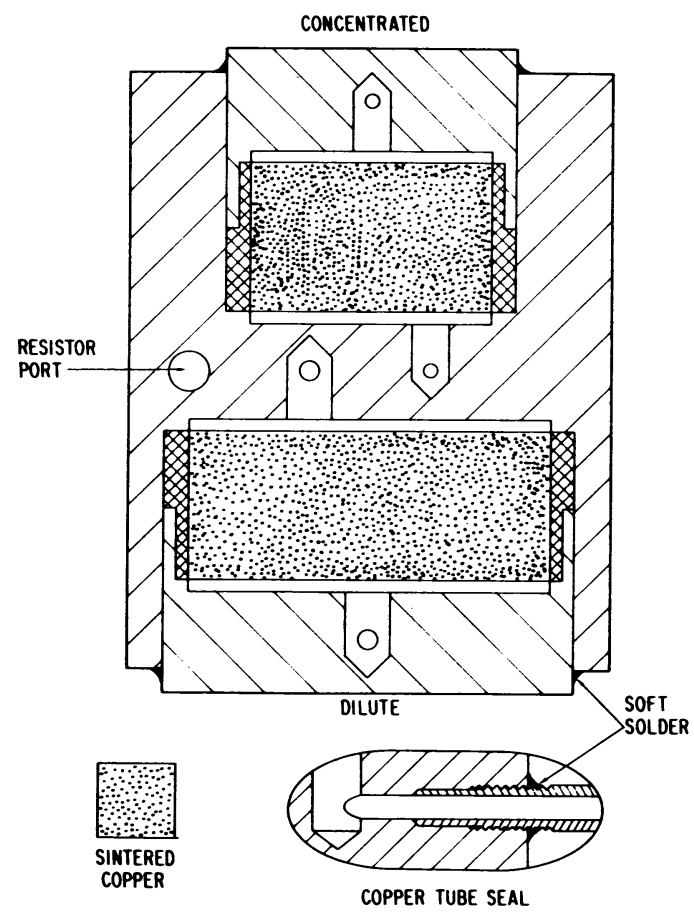

FIGURE 15

Cross-sectional diagram illustrating the construction of a typical heat exchanger for Refrigerator II. The hole at center left receives a Speer $1002 \mathrm{Mix,} 100 \Omega$, $1 / 4$ watt resistance thermometer. The inset illustrates the method of fabricating the copper tube connections used with the exchanger.

still bottom we proceed as follows [refer to the inset at the top of Fig. 14]. The cupronickel tube is selected and pressure tested for leaks at 60 to 70 atm. Flow impedance measurements are also made as indicated later to make certain that the tube is not plugged. The two outboard copper tubes are prepared by a slight counterbore about $5 \mathrm{~mm}$ deep. The cupronickel tube is inserted into the copper tube leading to the inner winding of the coil. It is spaced first by a brass bushing about $2 \mathrm{~mm}$ long and then by a bushing of hard solder machined from stock wire solder. The end is then fluxed and a small flow of helium caused to pass through the cupronickel tube to keep it clean during soldering. A small hot flame at the tip of the copper tube is sufficient 
to make the joint. The still bottom is next placed in the lathe and the still exchanger coil wound on the spool. The loose end is then inserted in the other outboard tube and soldered in exactly the same way as the other end. The cupronickel tube where it emerges from the copper tube is soft and weak, so it is cut off. Joints to other tubes are made by means of a brass coupling. The heater consists of a length of $0.05 \mathrm{~mm}$ dia. Evanohm [47] wire glued on cloth with General Electric 7031 varnish and placed in the bottom of the recess shown. Leads of $0.25 \mathrm{~mm}$

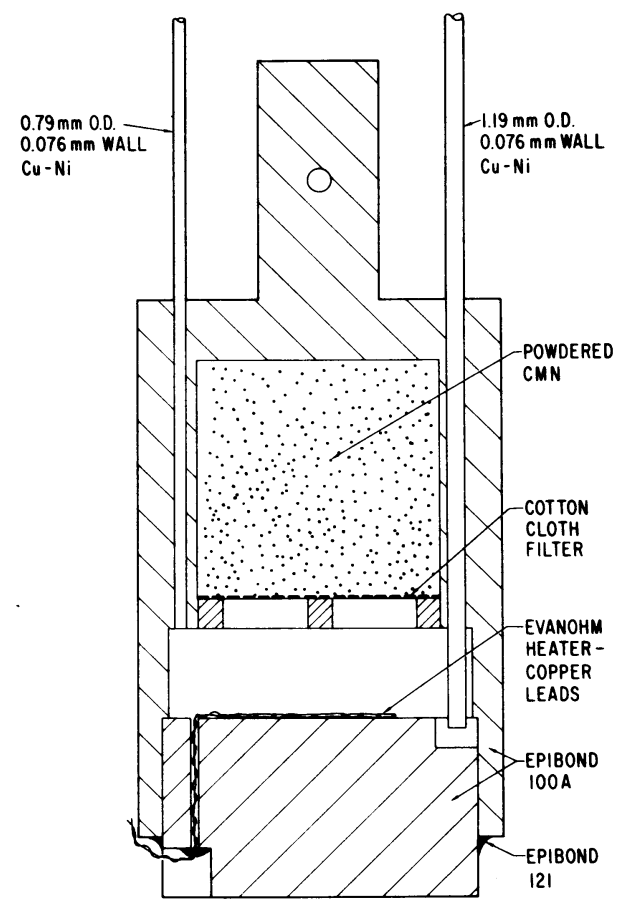

FIGURE 16

Cross-sectional view of an epoxy mixing chamber showing typical arrangement of parts.

diameter Quadruple Formex insulated copper wire are hard soldered to the Evanohm by a dipping technique. These then pass through one of the copper tubes, the vacuum seal being made using Emerson and Cuming' s Stycast 2850 GT epoxy. Care is taken to protect the leads from mechanical damage where they emerge from the epoxy. The still bottom is now ready to be soft soldered to the still top. The mechanical design shown in the cutaway enables a solder fillet to be obtained readily and minimizes the possibility of soft solder flowing into the heat exchange coil. The epoxy joint seems to be able to survive many remeltings of the soft solder.

The still top has been the subject of an extensive investigation. As we indicated in 


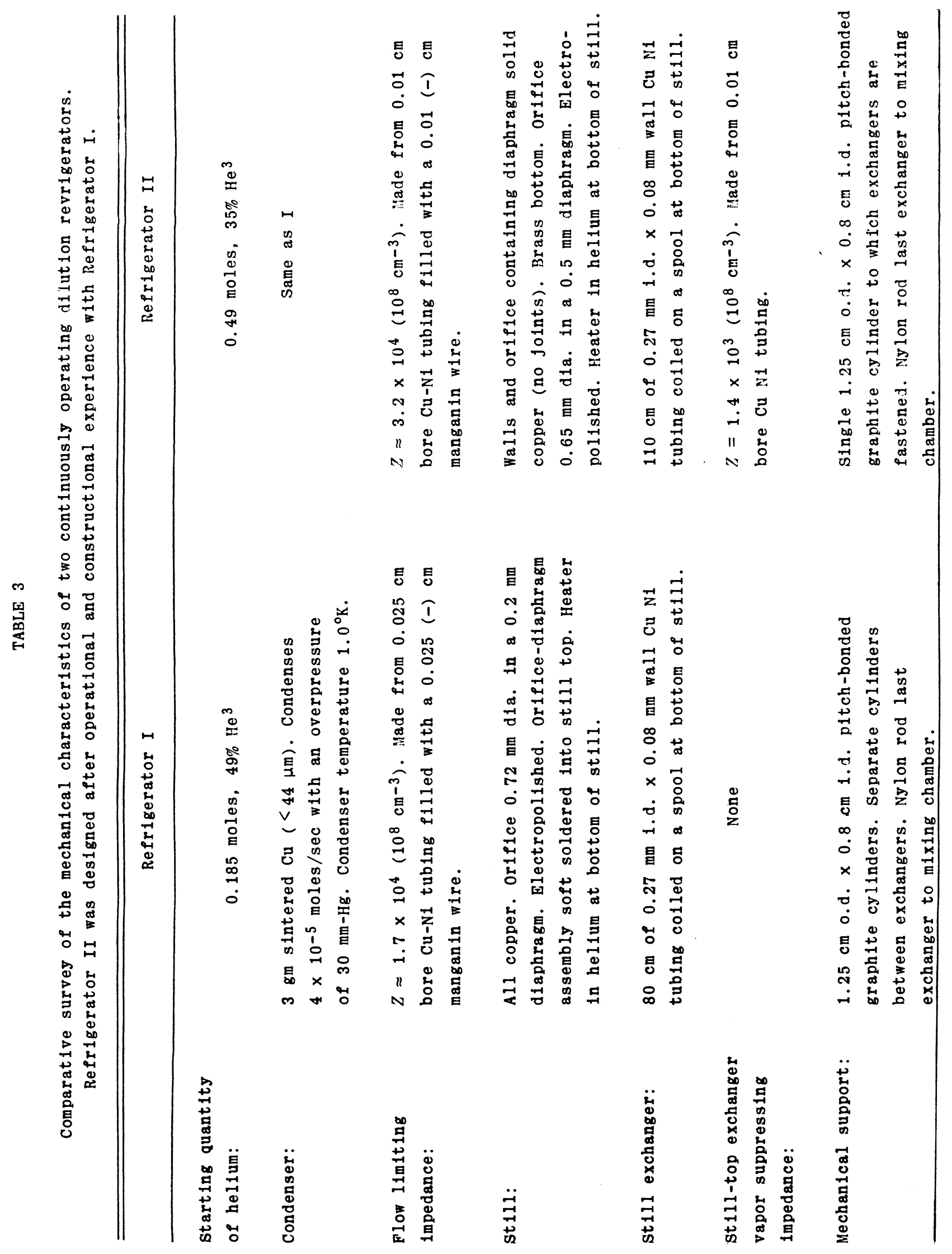




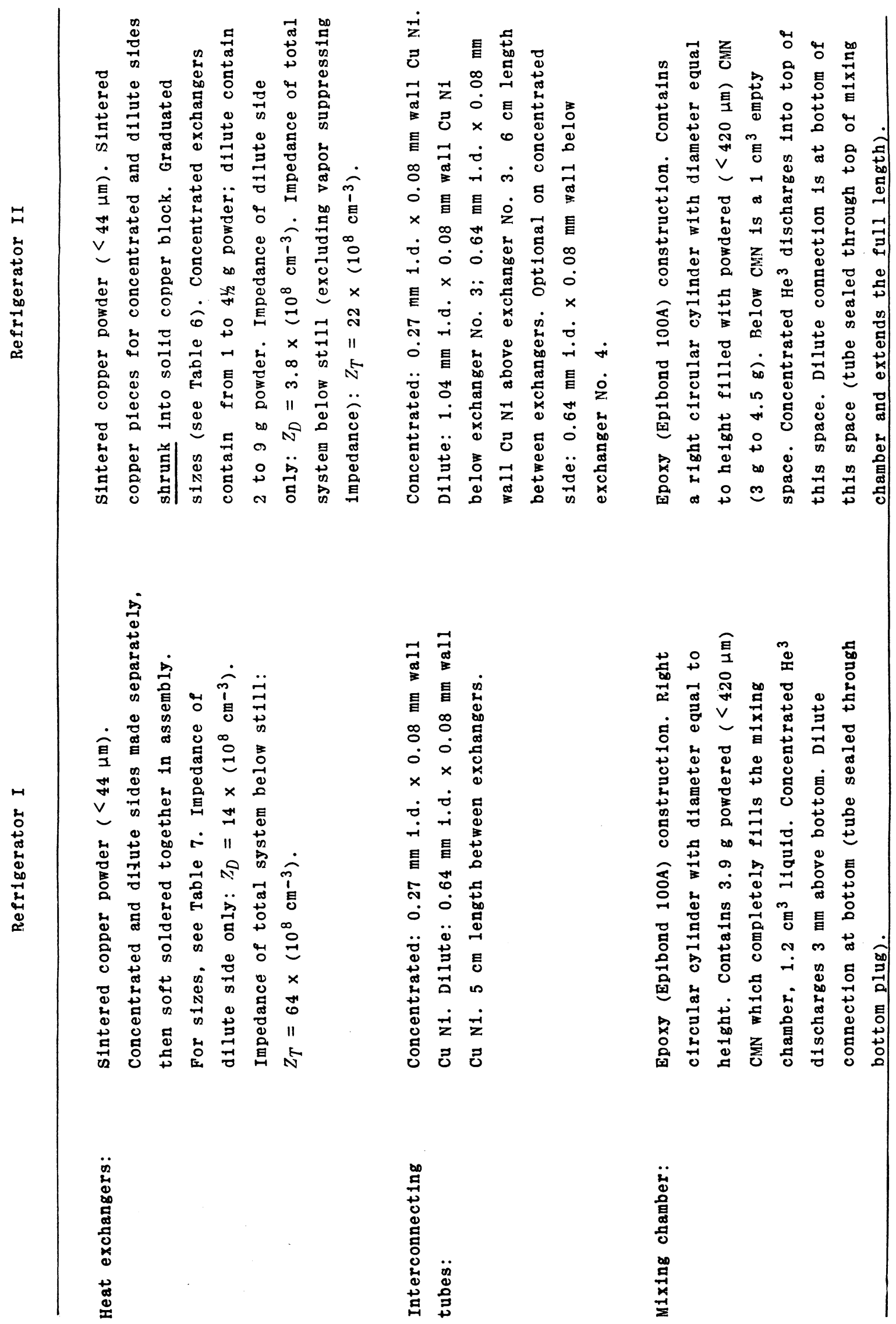


Section VI, it is very important to reduce the amount of He 4 circulated. Following the general guidelines presented there we performed a series of seven experiments with no apparatus at all below the still but with the output of the still exchanger connected directly to the dilute phase return inlet to the still. The condenser and main flow impedance were connected for normal operation. The circulated helium charge contained only about $10 \% \mathrm{He}^{3}$ so that, concentrated $\mathrm{He}^{3}$ dead volumes considered, the still would have a low concentration of $\mathrm{he}^{3}$ in it to simulate actual operation. The actual $\mathrm{He}^{3}$ concentration in the still fluid was unknown. The general characteristics of the still top and the results of the experiments are given in Table 4 . In experiment $A$ the still top, entirely copper and with a rather large $(1 \mathrm{~mm})$ chemically polished orifice, was filled with copper screen discs to eliminate any possible entrainment of $\mathrm{He}^{4}$ in the vapor stream as a result of splashing. The orifice was bright, but did not have a polished aspect. This arrangement was our first guess of a good still top design on the basis of earlier work. In experiment $B$ we then changed to brass still walls which were relatively poor thermal conductors compared with copper, halved the diameter of the orifice, and made the orifice in a thin sheet of copper foil which was electropolished before being soft soldered into the still top. No copper discs were used. The results in Table 4 show that whereas the still operating temperatures were about the same the $\mathrm{He}^{3 / \mathrm{He}^{4}}$ ratio was reduced substantially. Without making any other changes, in experiment $\mathrm{C}$ the orifice diameter was nearly doubled. Operating temperatures decreased by the $\mathrm{He}^{3} / \mathrm{He}^{4}$ ratio decreased further to a value about half that obtained in experiment $A$. Without changing orifice diameter substantially and still using the copper foil diaphragm we then changed back in experiment $D$ to copper walls. There was no change in the still operating temperature for a given power dissipation, but there was a marked improvement in $\mathrm{He}^{3 / \mathrm{He}^{4}}$ ratio. We then removed the still top and, making no other changes, filled the still top for experiment $E$ with copper screen discs. Examination of Table 4 shows that these had no effect on the $\mathrm{He}^{3} / \mathrm{He}^{4}$ ratio. This is a reasonable result, as it is to be expected that the still superfluid would vaporize quietly. Next we made the still top entirely of copper in experiment $F$ and increased substantially the diaphragm thickness while making an orifice of intermediate diameter. A further improvement of the $\mathrm{He}^{3 / \mathrm{He}^{4}}$ ratio, back to values similar to those obtained in experiment $A$, was obtained. In experiment $G$ the still bottom, which by this time looked somewhat the worse for wear, was changed for a new one. A further improvement was observed. From these experiments we conclude that both the still top and the diaphragm should be of relatively thick copper. The basis for this rule may be seen by comparing the results of experiments $A, C$, and $D$ since the orifice diameter was about the same for each one. Since the electropolish of the orifices in experiments $C$ and $D$ was much better than the chemical polish for the orifice in experiment $\mathrm{A}$ we expect less $\mathrm{He}^{4}$ film flow for experiments $\mathrm{C}$ and $\mathrm{D}$. Moreover in experiment $\mathrm{C}$, where the worst results were obtained, the still temperature was lowest; so that the equilibrium $\mathrm{He}^{\$} / \mathrm{He}^{4}$ ratio should have been highest. A simple explanation of these phenomena is obtained, if they are assumed to arise from the heat flow due to conduction and radiation, but probably not convection, coming down the pumping line. The extra $\mathrm{He}^{4}$ in experiments $C$ and $D$, at least, comes from $\mathrm{He}^{4}$ in the film adhering to the top and sides of the still which is evaporated due to local heating and then entrained in the vapor moving out the orifice. It was not sufficient in reducing the effect to make the walls of copper. The $0.05 \mathrm{~mm}$ copper diaphragm was particularly susceptible to local heating over the equilibrium still temperature not only because of its thinness but also because it was soft-soldered in, and soft solder is a very poor thermal conductor. It is not possible to say that there are no vestiges of this effect in the present still, but the analysis presented in a later section of operational data for the still in the actual experiments leads one to conclude that the unwanted $\mathrm{He}^{4}$ flow rates are rather reasonably interpreted as being due to film flow.

b. Heat exchangers. Many possibilities exist for the construction of heat exchangers of the type suggested in Sections $V$ and VII. There is still a need for simplification of construction, conservation of space, and reduction of flow impedance factor in the lowest temperature stages. Such improvements are being actively investigated, but for the present we will describe the 


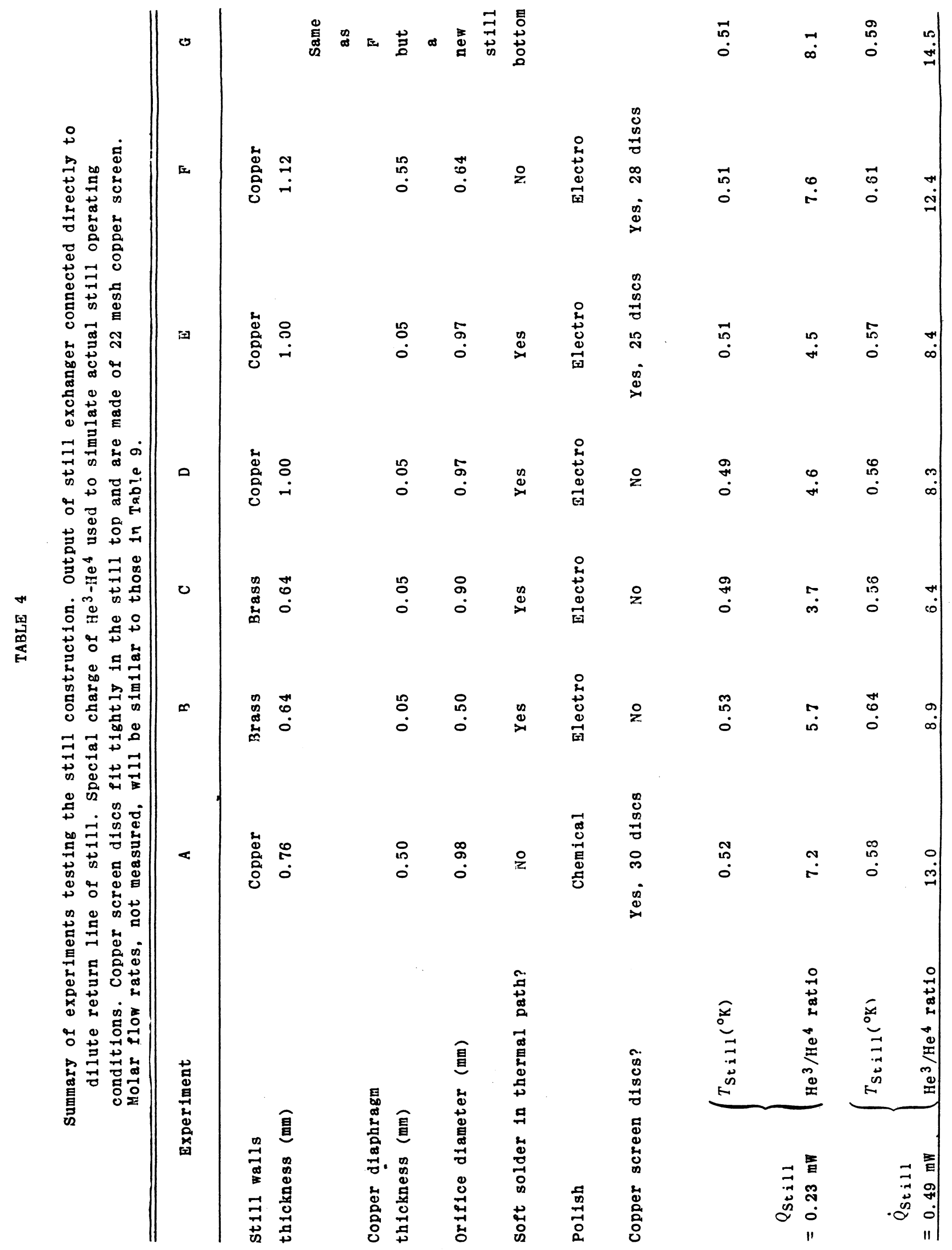


exchangers being used in Refrigerator II. These are shown as units assembled into the refrigerator in Fig. 14. A typical exchanger is shown in cross section in Fig. 15.

Preparation of the sintered copper is important. Exploratory work in this area was performed in collaboration with Mr. Gösta Ehnholm and Mr. David hamblen. The present exchangers use copper powder [48] with particle size less than $44 \mu \mathrm{m}$ and of $99.9 \%$ purity. Experiments were performed on the length of time in the furnace, the sintering temperature, the electrical resistivity, the flow impedance factor, and the effect of packing pressure. The copper is packed into oFHC copper tubes. Reasonable results are obtained with a small packing force and with a sintering time of one hour at $800^{\circ} \mathrm{C}$ in a reducing atmosphere provided by packing the powder filled copper tubes in charcoal. Recent work of Ennholm and W.C. Black indicates that better results may be obtained using a hydrogen atmosphere and a $700^{\circ} \mathrm{C}$ sintering temperature. To obtain a long cylinder of sintered copper from which pies for the individual exchangers could be readily parted off the copper tubes were filled in layers with powder. Referring to the sizes given in Table 5, we used $8 \mathrm{~g}$ per layer for the largest bore, $4 \mathrm{~g}$ for the next largest, and $1 \mathrm{~g}$ and $1 / 2 \mathrm{~g}$

TABLE 5

Characteristics of copper powder $(<44 \mu \mathrm{m})$ sintered for one hour at $800^{\circ} \mathrm{C}$ in $\operatorname{copper}$ tubes. The packing force is near the minimum necessary to keep the powder in the tube once the pistons are removed. $L$ is the diameter of the sintered copper and $l$ is its length. $Z$ is the flow impedance factor of the sintered copper cylinder as defined in equations $(61)$ and $(77)$. $z$ is a "resistivity" defined by $z=Z \frac{\pi D^{2}}{4 l}$. F is the fraction of the volume occupied by copper.

\begin{tabular}{|c|c|c|c|c|c|c|c|}
\hline $\begin{array}{l}\text { Sample } \\
\text { No. }\end{array}$ & $\begin{array}{l}\text { Packing force } \\
(k g-w t)\end{array}$ & $\begin{array}{c}D \\
(\mathrm{~cm})\end{array}$ & $\begin{array}{c}l \\
(\mathrm{~cm})\end{array}$ & $\begin{array}{l}\text { Mass } \\
(\mathrm{g})\end{array}$ & $\begin{array}{c}Z \\
\left(10^{8} \mathrm{~cm}^{-3}\right)\end{array}$ & $\left(10^{8} \mathrm{~cm}^{-2}\right)$ & $\begin{array}{c}F \\
(\%)\end{array}$ \\
\hline 1 & 1000 & 1.905 & 4.91 & 62. & 3.63 & 2.1 & 48.8 \\
\hline 2 & 1000 & 1.905 & 4.94 & 63.3 & 3.91 & 2.3 & 49.5 \\
\hline 3 & 400 & 1.27 & 5.64 & 32. & 6.61 & 1.5 & 49.4 \\
\hline 4 & 400 & 1.27 & 4.85 & 26.8 & 6.24 & 1.6 & 47.2 \\
\hline 5 & 100 & 0.953 & 3.41 & 10.0 & 9.65 & 2.0 & 44.5 \\
\hline 6 & 100 & 0.953 & 3.48 & 10.2 & 8.57 & 1.8 & 44.5 \\
\hline 7 & 100 & 0.953 & 3.54 & 10.3 & 10.0 & 2.0 & 44.1 \\
\hline 8 & 50 & 0.635 & 3.54 & 4.3 & 17.1 & 1.6 & 39.0 \\
\hline 9 & 50 & 0.635 & 3.42 & 4.3 & 19.4 & 1.9 & 41.3 \\
\hline 10 & 50 & 0.635 & 3.36 & 4.3 & 16.7 & 1.6 & 42.9 \\
\hline
\end{tabular}

for the two smallest bores. After sintering at $800^{\circ} \mathrm{C}$ the sintered copper mass sometimes tends to shrink away from the walls, so the copper tubes are swaged in a lathe collet after the sintering to compress the walls tightly to the powder. Characteristics are given in Table 5. The flow impedance factor $Z$ is measured in a simple experimental arrangement in which helium or nitrogen gas is caused to flow through the specimen by means of a measured pressure increment $\Delta P$ over atmospheric pressure. The effluent gas is caused to pass just under the surface of a water bath and the volume rate of flow $V$ obtained by means of a stop watch and an inverted graduated cylinder initially filled with water. For helium gas at room temperature the flow 
impedance factor is calculated from

$$
Z=\frac{\left(1+\frac{\Delta ?}{2 P}\right) \Delta P}{14.5 \dot{V}} \frac{\left(\mathrm{cm}^{3} / \mathrm{sec}\right)}{\mathrm{mm}-\mathrm{Hg}} \times 10^{8} \mathrm{~cm}^{-3},
$$

where $P_{a}$ is atmospheric pressure. The flow "resistivity" factor of the sintered copper is reasonably reproducible, though it would be highly desirable to reduce it by at least another factor of ten. Further details of the above as well as a discussion of electrical measurements are given elsewhere [49].

The sections of sintered copper encased in copper tube are installed in the copper block shown in Fig. 15 by means of a series of expansion fits. The main block is first prepared by boring and counterboring as shown. Holes for the exit tube connections are drilled - and tapped where necessary. Slots for the exit tube connections are milled at the ends. The plug containing the sintered copper is made by first facing the sintered copper in its copper case to the desired length, then turning it back about half its length, and finally making an expansion fit to the previously bored, counterbored, and drilled copper plug. The expansion fit is made in the lathe by fastening the solid copper plug in a collet chuck while locating the sintered copper piece in a holder held in the tailstock. The sintered copper is then cooled to liquid nitrogen temperature in an aluminum foil boat. The boat is then torn away and the sintered copper piece pushed into the copper cup. Generally 0.01 to $0.02 \mathrm{~mm}$ of interference are allowed for this type of fit. The end plug is left oversize on the outside diameter for the above operations. It is then turned to have the desired interference with the main body and a second expansion fit made in the same way as indicated above.

Connection of the body to copper tubes is made as shown in the inset in order to avoid any possibility that soft solder used to seal the joints would plug the tubes. Copper refrigeration capillary tubes are used for this purpose. A die is used to thread the end of the tube, and the tube is then turned back a short distance to essentially the tap drill diameter. The body is prepared to receive this piece as shown. When the copper tube is screwed in, it makes a cold joint so that the soft solder is restricted to the locations shown. The expansion fits of the end plugs in the main body similarly act to prevent the entrance of soft solder when these plugs are soldered. All soldering is done by iron rather than by torch. The copper tube size most generally used is $2.1 \mathrm{~mm} \mathrm{o.d.} \mathrm{and} 0.79 \mathrm{~mm} \mathrm{i.d.} \mathrm{However,} \mathrm{in} \mathrm{the} \mathrm{coldest} \mathrm{two} \mathrm{exchangers} \mathrm{the} \mathrm{con-}$ nections to the dilute side are made with copper tubes $2.8 \mathrm{~mm}$ o.d. and $1.27 \mathrm{~mm} \mathrm{i.d.}$

In Table 6 we give some characteristics of exchangers used in Refrigerator II. For comparison we give in Table 7 some of the characteristics of the exchangers of Refrigerator I, which were of a more rudimentary construction. Inspection of the data given in these tables indicates that in II a substantial increase in volume and decrease in impedance were obtained relative to the exchangers in $I$.

c. Mixing chamber. We have used a variety of mixing chambers constructed of both copper and epoxy. The copper mixing chambers are provided with sintered copper to enable heat to be transferred to the outside readily. They can be provided with a variety of auxiliary features such as thermal grounding devices, mechanical thermal contact fixtures, etc. However, we have not used them for quantitative work since we are unable to do accurate thermometry without immersing cerium magnesium nitrate (CMN) in the helfum itself. Eddy currents in the copper make it impossible to make good mutual inductance measurements and also lead to a heating problem. Hence we have for years used epoxy resins in our low temperature apparatus, in particular, the $150^{\circ} \mathrm{C}$ curing Epibond $100 \mathrm{~A}$ and the room temperature curing Epibond 121 [50]. Many details of 
TABISE 6

Summary of data for heat exchangers used in Refrigerator II. The copper powder has a maximum grain size of $44 \mu \mathrm{m}$. The higher the exchanger number, the lower 1 ts onerating temperature.

\begin{tabular}{|c|c|c|c|c|c|c|c|c|c|c|}
\hline \multirow{2}{*}{$\begin{array}{c}\text { Exchanger } \\
\text { No. }\end{array}$} & \multicolumn{2}{|c|}{ Copper powder volume } & \multicolumn{2}{|c|}{ Hellum volumea,b } & \multicolumn{2}{|c|}{ Cross-section } & \multicolumn{2}{|c|}{ Length } & Measured & Impedance \\
\hline & $\begin{array}{l}\text { Dilute } \\
\left(\mathrm{cm}^{3}\right)\end{array}$ & $\begin{array}{l}\text { Conc. } \\
\left(\mathrm{cm}^{3}\right)\end{array}$ & $\begin{array}{l}\text { Dilute } \\
\left(\mathrm{cm}^{3}\right)\end{array}$ & $\begin{array}{l}\text { Conc. } \\
\left(\mathrm{cm}^{3}\right)\end{array}$ & $\begin{array}{l}\text { Dilute } \\
\left(\mathrm{cm}^{2}\right)\end{array}$ & $\begin{array}{l}\text { Conc. } \\
\left(\mathrm{cm}^{2}\right)\end{array}$ & $\begin{array}{l}\text { D1lute } \\
\text { (cm) }\end{array}$ & $\begin{array}{l}\text { Conc. } \\
(\mathrm{cm})\end{array}$ & $\begin{array}{r}\text { Dilute } \\
\left(10^{8} \mathrm{~cm}^{-3}\right)\end{array}$ & $\begin{array}{l}\text { Conc. } \\
\left(10^{8} \mathrm{~cm}^{-3}\right)\end{array}$ \\
\hline 1 & 0.25 & 0.125 & 0.26 & 0.16 & 1.27 & 0.71 & 0.41 & 0.33 & 0.73 & 1.24 \\
\hline 2 & 0.50 & 0.25 & 0.52 & 0.27 & 2.85 & 1.27 & 0.36 & 0.41 & 0.35 & 0.71 \\
\hline 3 & 0.75 & 0.375 & 0.77 & 0.40 & 2.85 & 1.27 & 0.53 & 0.32 & 0.46 & 0.98 \\
\hline 4 & 1.00 & 0.50 & 1.03 & 0.54 & 2.85 & 1.27 & 0.71 & 0.32 & 0.83 & 0.85 \\
\hline 5 & 1.00 & 0.50 & 1.03 & 0.54 & 2.85 & 1.27 & 0.71 & 0.82 & 0.64 & 1.22 \\
\hline
\end{tabular}

\footnotetext{
Calculated from average characteristics of the sintered copper cylinders.
} 
construction practice relevant to the present work may be found in Ref. 46 .

A typical mixing chamber used in the present work is shown in Fig. 16. This mixing chamber is provided with powdered CMN for temperature measurements $[51,54]$. The shape of the CMN is a right circular cylinder with diameter equal to height. It is separated from the lower part of the mixing chamber by a cotton cloth filter glued with General Electric 7031 varnish to an

\section{TABLE 7}

Summary of data for heat exchangers used in Refrigerator 1 . The copper powder has a maximum grain size of $44 \mu \mathrm{m}$. The higher the exchanger number, the lower its operating temperature. The impedances of exchangers 3 and 4 are believed to be spuriously low as a result of imperfect contact of sintered copper with the copper walls.

\begin{tabular}{|c|c|c|c|c|c|c|}
\hline \multirow{3}{*}{$\begin{array}{l}\text { Exchanger } \\
\text { No. }\end{array}$} & \multicolumn{2}{|c|}{ Copper powder volume } & \multicolumn{2}{|c|}{ Cross-section } & \multicolumn{2}{|c|}{ Measured impedance } \\
\hline & Dilute & Conc. & Dilute & Conc. & Dilute & Conc. \\
\hline & $\left(\mathrm{cm}^{3}\right)$ & $\left(\mathrm{cm}^{3}\right)$ & $\left(\mathrm{cm}^{2}\right)$ & $\left(\mathrm{cm}^{2}\right)$ & $\left(10^{8} \mathrm{~cm}^{-3}\right)$ & $\left(10^{8} \mathrm{~cm}^{-3}\right)$ \\
\hline 1 & 0.168 & 0.084 & 0.712 & 0.400 & 4.6 & 3.7 \\
\hline 2 & 0.224 & 0.112 & 0.712 & 0.400 & 2.6 & 4.6 \\
\hline 3 & 0.224 & 0.112 & 0.712 & 0.400 & 1.1 & 2.6 \\
\hline 4 & 0.224 & 0.112 & 0.712 & 0.400 & 0.8 & 2.7 \\
\hline
\end{tabular}

epoxy frame. The quantity of $\mathrm{He}^{3}$ in the system is adjusted so that the phase boundary occurs in the space below the CMN. This has been arranged so that no flow of $\mathrm{He}^{3}$ occurs through the CMN powder since this can lead to viscous heating. The heater used to control the mixing chamber temperature is located on the top of the epoxy end plug. Both the end plug and the electrical leads are sealed with Epibond 121. All the rest of the epoxy work is with Epibond 100A. The concentrated $\mathrm{He}^{3}$ inlet tube is the $0.79 \mathrm{~mm}$ o.d. cupronickel tube on the left. The $\mathrm{He}^{3}$ in the dilute phase moves through the $1.19 \mathrm{~mm} 0 . \mathrm{d}$. tube on the right.

The mixing chamber is attached to the rest of the dilution refrigerator by the pinned joint shown in Figs. 14 and 16. To complete the connections the two brass couplings shown in Fig. 14 are soft soldered into position. We would like to emphasize that these operations are very simple indeed, so simple that we feel that in many applications of the dilution refrigerator one should simply change experiment or experimental sample by changing the mixing chamber. That is, we specifically recommend against copper mixing chambers unless there is no other recourse and recommend for epoxy mixing chambers, which can be made readily in a great variety of forms and can moreover carry their own thermometer. It is generally possible to alter the mixing chamber by boring out the end plug, leaving the main body unchanged, making appropriate changes, and then resealing a new end plug with Epibond 121.

d. Condenser and main impedance. The location of the condenser and main impedance are 
indicated on Fig. 17. These two components are designed together. The condenser contains $3 \mathrm{~g}$ of sintered copper powder sealed into a copper cylinaer which is greased into a hollow cylinder passing through the $\mathrm{He}^{4}$ evaporator. In a separate experiment in which helium was allowed to enter the condenser from a known volume the rate of decrease of pressure was used to determine the condensation rate for this condenser for a $\mathrm{He}^{4}$ evaporator temperature of about $1^{\circ} \mathrm{K}$. It was

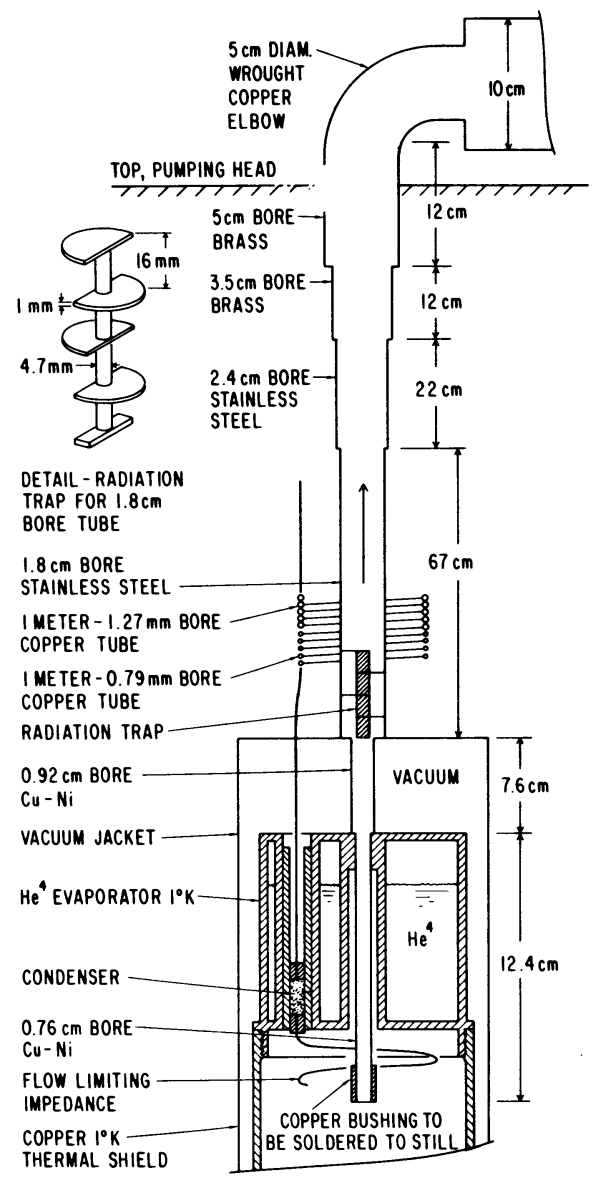

FIGURE 17

Schematic diagram of the dilution refrigerator system within the cryostat. All parts of the dilution refrigerator below and including the still, shown in Fig. 14, are omitted from this diagram for clarity. The general configuration of the He 4 evaporator and the copper $1^{\circ} \mathrm{K}$ thermal shield as they relate to the dilution refrigerator are also shown. The stainless steel tubes in the pumping lines have a wall thickness of $0.5 \mathrm{~mm}$; the cupronickel (70-30) tubes have a wall thickness of $0.15 \mathrm{~mm}$. The copper bushing on the bottom of the pumping line is hard soldered to the cupronickel and soft soldered into the counterbore shown at the top of the still in Fig. 14. The flow limiting impedance tube is inserted in the coupling leading upward from the still exchanger, Fig. 14, and soft soldered. The radiation trap, shown in detail at the upper left, is coarsely blackened and fust slides into the bore of the main pumping line. 
found that for a condensing pressure of $30 \mathrm{~mm}-\mathrm{Hg} \mathrm{He}^{3}$ condensed at a rate of $4 \times 10^{-5} \mathrm{moles} / \mathrm{sec}$. If now the main impedance is such that at a pressure of $30 \mathrm{~mm}-\mathrm{Hg}$ it will pass more than this quantity of'liquid, the condenser will operate empty. Enough vapor will pass the impedance to build up the pressure to the operating value. It is undesirable to pass vapor to the still exchanger, so the main impedance is designed to require a substantially greater pressure than $30 \mathrm{~mm}-\mathrm{Hg}$ to pass a liquid flow rate of $4 \times 10^{-5} \mathrm{moles} / \mathrm{sec}$. In this case the condenser will operate full of liquid. For design purposes we assume that the viscosity of $\mathrm{He}^{3}$ is [53] $30 \times 10^{-6}$ dy sec/ $\mathrm{cm}^{2}$ at $1{ }^{\circ} \mathrm{K}$, the condenser temperature. Using a molar volume of about $37 \mathrm{~cm}^{3} / \mathrm{mole}$ the main impedance is designed from equation (61) for a $\Delta P$ about $1 \frac{112}{2}$ to 2 times in excess of the pressure required to keep the condenser full. The impedance is made using standard lengths of cupronickel tubing of either $0.27 \mathrm{~mm}$ or $0.1 \mathrm{~mm} 1 . \mathrm{d}$. by inserting into the tube a tightly fitting length of manganin wire. The impedance factor $Z$ is then measured and the tube cut to the desired length in accord with this measurement.

$e$. Interconnections. The sizes, lengths, and arrangement of interconnecting tubes at high temperatures are determined by the problems of thermal conduction and convection on the dilute side and by the requirement that no vapor be allowed to form on the concentrated side. The thermal conduction problem in the presence of flowing $\mathrm{He}^{3}$ is not serious. This may be seen in terms of a simple analysis in which the thermal conductivity of the dilute phase is assumed constant and the dilute phase enthalpy function $h$ is assumed equal to $5 / 2 k T$. Viscous heating is negligible, so heat balance leads to the following equation

$$
\frac{5}{2} \dot{n}_{3} k \frac{d T}{d l}=\frac{\pi}{4} d^{2} \kappa_{d} \frac{d^{2} T}{d l}
$$

for the variation of temperature with length $l$ along an interconnecting tube of diameter $d$. The boundary conditions on this equation would set, for example, the temperature at one end equal to the temperature of the first exchanger and would let the total rate of heating in the tube be equal to the net rate at which heat is conducted in through the ends. For the assumptions given the temperature varies exponentially with length. The characteristic length may be readily seen from equation (78) to be

$$
l_{0}=\frac{\frac{\pi}{4} d^{2} \kappa_{d}}{\frac{5}{2} \dot{n}_{3} k} .
$$

Assuming $x \approx 0.01$ in equation (16) we find numerically

$$
l_{0} \cong 2\left(\frac{d}{1 \mathrm{~mm}}\right)^{2}\left(\frac{10^{-5} \mathrm{moles} / \mathrm{sec}}{\dot{n}_{3} / A_{\mathrm{n}}}\right) \mathrm{mm} .
$$

This distance is so small with respect to the actual distances between exchangers, for values of $d$ and $\dot{n}_{3}$ in practical use, that we can assume thermal conduction along the interconnecting tubes at high temperatures to be negligible. This result is in agreement with experimental results mentioned at the beginning of Section IX where no temperature gradient along the interconnecting tubes could be detected. The diameter and arrangement of these tubes is then selected to avoid convection. The values given in Table 3 and the geometrical arrangement shown in Fig. 14 are satisfactory.

The tube interconnecting the still and the first exchanger on the concentrated side may be required to have a relatively large impedance if vapor flow through this tube is to be suppressed. 
In Refrigerator II with a still power of about $1 \mathrm{~mW}$ the still temperature rises to about $0.82^{\circ} \mathrm{K}$. At this temperature the vapor pressure of $\mathrm{He}^{3}$ is about $3.2 \mathrm{~mm}-\mathrm{Hg}$. Unless the total pressure rise from the still on the dilute side to the still on the concentrated side is in excess of this pressure, no liquid will be present in the still exchanger. Vapor will then tend to flow out of the still exchanger toward the first heat exchanger and the pressure drop will adjust itself naturally. The heat influx to the first exchanger will be profoundly increased with a resultant harmful effect on the operation of the whole refrigerator. The required pressure rise is obtained through the effect of viscosity, equation (61). For Refrigerator II the value of $Z$ given in Table 3 is adequate to make the pressure $5 \mathrm{~mm}-\mathrm{Hg}$ for a molar flow rate of $4 \times 10^{-5} \mathrm{moles} / \mathrm{sec}$. This is enough to keep the still exchanger full of liquid. So far as the tubes interconnecting the lower exchangers are concerned, we note that even for a temperature as high as $0.5^{\circ} \mathrm{K}$ the vapor pressure of $\mathrm{He}^{3}$ is equivalent to the hydrostatic pressure of a $3 \mathrm{~cm}$ high column of $\mathrm{He}^{3}$. Hence ordinary hydrostatic pressure differences should solve any possible problems.

Interconnecting tubes at low temperatures can in principle lead to problems with viscous heating, referring to equations (67) and (58) and the accompanying discussion. In practice there does not seem to be much sense in designing the tubes so that viscous heating in them is very much smaller than that in the exchangers. For that matter, it will not help to reduce viscous heating in the concentrated side if heating is excessive in the dilute side. For the parameters given under II in Table 3 the dilute side interconnections are very conservative while the concentrated ones are not, as may be verified by substituting in equation (62) and comparing with the figures given for $Z$ in Table 6 . However, the optional lower impedance tubes for the concentrated side mentioned in Table 3 do not improve the operation since it is probable that the limiting factor is already viscous heating on the dilute side.

To conclude this section we wish to emphasize the practical importance of using cupronickel $(70-30)$ for the interconnections. This alloy may be soldered readily using the ordinary $\mathrm{Sn}-\mathrm{Pb}$ solder containing a core of flux frequently used in work in electronics. It has an excellent thermal resistance. We have experienced no problem with corrosion. There are so many joints in a device of this type that it must be a simple matter to make joints easily and with confidence. We therefore wish to argue against the use of stainless steel tubing. Further, in making joints one must be very cautious about plugging these fine bore tubes. Plugging can be avoided on assembly by slipping one tube some distance inside another before making a joint or by using long couplings whose central part is cooled with a bit of moisture on a cotton pad during soldering. It can be avoided on disassembly by having some excess pressure in the apparatus and by unsoldering downstream parts first.

$f$. Hechanical support. The use of pitch-bonded graphite as a mechanical support for work below $1^{\circ} \mathrm{K}$ was suggested by Shore, Sailor, Marshak, and Reynolds [54]. Extrapolating the work of Berman [55] one would find the thermal conductivity of such graphite to be approximately $40 \mathrm{~T}^{2.6} \mathrm{erg} / \mathrm{sec} \mathrm{cm} \mathrm{K}{ }^{03.6}$. The thermal conductivity below $1^{\circ} \mathrm{K}$ would accordingly be very much less than that of other amorphous solids [56], including teflon and nylon, frequently in use as low temperature support material. Graphite has the advantage of a small expansion coefficient, probably less than copper. It is, moreover, reasonably strong. So far as we know we do not have problems with it in regard to adsorption of $\mathrm{He}^{4}$ heat exchange gas, though we generally "bake out" our apparatus at about $10^{\circ} \mathrm{K}$ while pumping the helium exchange gas.

In Refrigerator I the graphite supports were modular and screwed together using brass or copper unions. The thread was about $1 \mathrm{~cm}$ in diameter with about 6 threads per $\mathrm{cm}[3 / 8-16 \mathrm{NC}]$. In Refrigerator II the support is shown in Fig. 14. Details are given in Table 3. In this case one single column about $8 \mathrm{~cm}$ long was used with the four-exchanger arrangement. Connection to the graphite was again by the above type of thread. Location of the exchangers was alsj about as shown. The exchangers were supported from the graphite on the brass holders shown. In this 
connection we should remark that in the actual device radial space is used more effectively than shown on Fig. 14. The end of the collar away from the exchanger was milled off to leave a gap while some of the collar next to the exchanger was removed to enable it to rest closer to the graphite support column. The method of fastening the brass support to the graphite is not shown in Fig. 14. Fastening is achieved by a clamping action using a machine screw. One side of the brass collar is drilled and countersunk while diametrically opposite it the brass collar is tapped for the screw. Clearance holes are drilled in the graphite at the proper height and angle. The exchangers are fastened to the brass supports by small screws which fasten to tapped offcenter holes in the bottom end-plugs of the exchangers.

g. Testing. Two types of testing are important before the dilution refrigerator is assembled into the cryostat. These are leak and flow impedance testing. All tubes used in construction are pretested by pressurizing to 60 or 70 atm and looking for bubbles when they are submerged in water. Epoxy parts and heat exchangers are similarly tested at 10 to 20 atm. Tubes are assembled into the apparatus once it is mounted mechanically and then soldered using Ersin multicore [57] solder. These joints are made and then inspected with great care attending each joint, which is required to turn out with a shiny appearance and free from pores. All joints in the assembly below the still are then pressure tested at about 10 to 20 atm as above. The still itself is usually tested with a helium mass spectrometer leak detector since the pumping impedance is low. However, the pressure test is more effective than the vacuum test on the rest of the apparatus because of the high pumping impedance of the various parts. If the assumbly below the still passes the pressure test it is then assembled into a vacuum test can which is evacuated and connected to the leak detector. The dilution refrigerator assembly is then pressurized with He 4 for a final leak test, usually both at liquid $N_{2}$ temperature and room temperature. Only the last of the above leak tests is at all time consuming. It is the most sensitive. However, the pressure tests enable poor work to be discovered quickly.

Flow impedance testing is also important, not only to detect the possible plugging with solder of one of the small tubes but also to verify that the flow impedance factors are no larger than they should be. Of the difficulties we have experienced with these refrigerators, perhaps the most disastrous has been a spuriously high flow impedance factor on the dilute side causing an excessive osmotic pressure drop, a low $\mathrm{He}^{3} / \mathrm{He}^{4}$ ratio, and a limited refrigeration capability. The $Z$ factor is measured for both sides of each exchanger, both at room and 1 iquid nitrogen temperatures, using helium gas which has been passed through Linde 13X "Molecular Sieven [58] to purify it. The assembled apparatus is also measured, sometimes at liquid $\mathrm{N}_{2}$ temperature and always at room temperature. Separate measurements of the dilute side through the mixing chamber and of the total circuit, Table 3, are made since $Z$ for the dilute side is relatively small.

In practice, the still without the exchanger assembly is usually soldered to the pumping system first and leak tested. Then the exchanger assembly is fastened to the bottom of the still with machine screws using the flange indicated in Fig. 14. Still and exchangers are interconnected and the joint between mixing chamber and the last exchanger on the concentrated side broken to allow flow measurements to this point. This joint is then remade as a final operation. When the mixing chamber is changed, for example to change experiments, the two couplings above the mixing chamber are unsoldered in position, and the mixing chamber is unpinned from the support rod and removed. In reassembly the dilute side coupling is always made first, a flow test is made, and finally the concentrated side coupling is soldered.

\section{B. Auxiliary components}

Aside from the dilution revrigerator components discussed above, a variety of other components 
of a rather standard nature are required to circulate the gas and to provide a suitable environment for the low-temperature device itself.

a. Cryostat components. A schematic diagram of the cryostat, exclusive of the still, exchangers, and mixing chamber, is given in Fig. 17. The pumping line specifications are adequate for the gas flow rates which can be handled by our pumping system. For a high throughput system the reader is referred to the work of Neganov, Borisov and Liburg [8]. In their system, the pumping line not only is larger than ours but also is kept at $\mathrm{N}_{2}$ temperature or below.

In mechanical construction pumping lines of stainless steel have hard soldered brass or copper bushings to facilitate soft soldering in assembly. Extreme care is used to avoid contaminating the stainless steel with chloride soft solder fluxes. The thermal radiation traps for the pumping lines are made from solid brass bar stock and have the form given in the inset in Fig. 17. Dimensions given there are for the dilution refrigerator pumping line, but the traps for other lines are similarly designed. These traps slide down the pumping lines and rest at the level of the flange above the vacuum jacket. They are strongly etched and then chemically blackened.

b. Room temperature components. A plumbing circuit which has sufficiently flexible characteristics is shown in Fig. 18. All valves and gauges shown have proved to be important. The volumes indicated for the storage containers are, of course, peculiar to the size of the helium charge for our system. A $25 \mathrm{~mm}$ i.d. line is used between the booster pump and the mechanical pump and a $10 \mathrm{~mm}$ i.d. line for the bypass, but otherwise at "high" pressures the lines are 3 to $4 \mathrm{~mm}$ i.d. Starting on the low pressure end (details of the pumping lines are given on the figure) the valving arrangement for the Leak Detector (Veeco MS9-A) allows both leak test and a positive shutoff for the needle valve, which is used to bleed some helium into the leak detector line for measuring the $\mathrm{He}^{3} / \mathrm{He}^{4}$ ratio. Valving is arranged to bypass the booster pump at high pressures. It is also possible to circulate gas by means of the mechanical pump through the trap system without admitting gas to the cryostat. The "oil" trap is $25 \mathrm{~mm}$ i.d. and $32 \mathrm{~mm}$ long and filled with copper screen. The molecular sieve trap is $32 \mathrm{~mm}$ i.d. and $42 \mathrm{~mm}$ long and filled with Linde $13 \mathrm{X}$ molecular sieve pellets. Valving and connections are arranged so that these traps may be heated with a heat gun with gas flowing through them for purging and cleaning. The 12.61. storage volıme may either be in the circuit or bypassed. Pressure measurements may be made with the Wallace and Tiernan gauges. Both pressures near $1 \mathrm{~atm}$ and lower pressures may be measured with some accuracy. Among its other uses the thermocouple vacuum gauge located as indicated on the "high" pressure side may be used to check for continuity of the "main impedance" by filling the vacuum lines above the still with helium gas and pumping out the "high" pressure side and this thermocouple gauge. The rate of rise of pressure than indicates whether or not the impedance is plugged.

During normal operation the $12.6 \mathrm{l}$. volume is bypassed so that if a plug were to develop in the high pressure line, pressures dangerous to the apparatus, particularly the mechanical pump, could occur. We have therefore incorporated the safety system shown at the left in Fig. 18. It is valved to allow evacuation and also to shut off a relief valve set to open for a pressure difference two or three times larger than the normal condensing pressure of the system while in steady operation. The volume of this system is selected so that even with the pressure increment of the relief valve the entire helium charge can be dumped without requiring the pressure above the mechanicos pump to exceed one atm. 


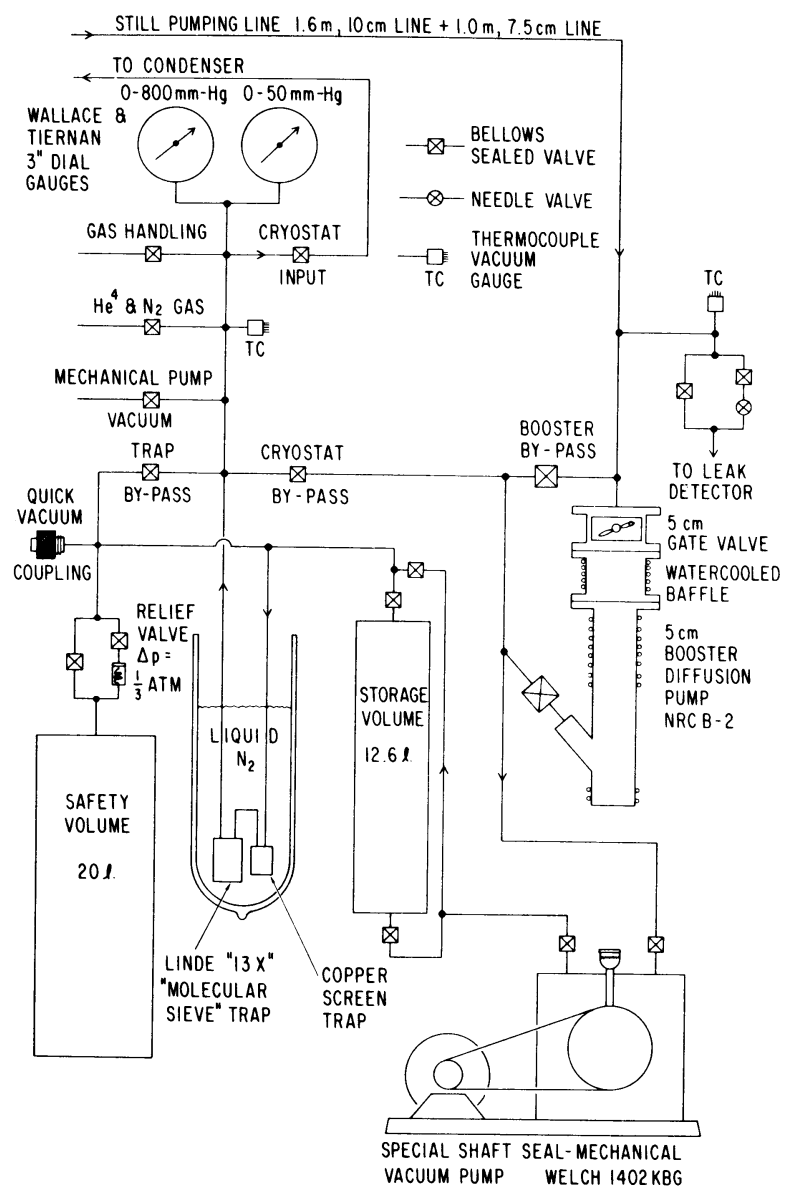

FIGURE 18

External gas circulation and handing system for the continuous dilution refrigerator. The gate valve is a Consolidated Vacuum Corporation (CVC) type VST-21. The baffle is a CVC W-4. The NRC B-2 booster diffusion pump is unfortunately no longer manufactured. Speed is required of this pump in the $10^{-2} \mathrm{~mm}-\mathrm{Hg}$ pressure range. Available pumps for this region, e.g. the CVC "KS-200", are much larger and more expensive than are needed for the present size of dilution refrigerator. The bellows-sealed valves are mostly Veeco Type FL 25?, though similar valves of $2.5 \mathrm{~cm}$ dia. are used in the main circuit. These valves are closed by a flat neoprene gasket. Similar valves with Viton $0-r i n g$ seals are unsatisfactory as a result of an excessive helium diffusion rate. The needle valve is an Ideal, Type 52-2-13. The leak detector is a Veeco MS9A modified according to Ref. 46 to allow measurement of $\mathrm{He}^{3} / \mathrm{He}^{4}$ ratios. The quick vacuum coupling may be used for purging the traps, while hot, with dry flowing gas from the external gas supply. The relief valve is a Circle Seal, Type 559B-2MP-5, set to operate on a 1/3-atm pressure difference. The dial pressure gauges are Wallace and Tiernan, Type FA 160 with a 3-in. dial. The 1ine labeled "Gas Handling" leads to a system where $\mathrm{He}^{3}-\mathrm{He}^{4}$ mixtures may be prepared or purified. During normal operating conditions valves to the storage and safety volumes are closed; the gas circulates as shown by the arrows. When the refrigerator is left unattended the bellows-sealed valve above the relief valve in the safety system is opened. 


\section{Dilution Refrigerator Operation}

\section{A. Starting up}

The amount of $\mathrm{He}^{3}$ and $\mathrm{He}^{4}$ in the starting helium charge is determined from volume measurements or estimates of the volumes of the various components of the refrigerator. The amount of $\mathrm{He}^{3}$ must be enough to fill the entire concentrated side, including the mixing chamber. There must be enough $\mathrm{He}^{4}$ to fill the entire dilute side and to fill the still up substantially above the top of the still exchanger. The still is usually designed so that it can hold the entire liquid charge itself. In view of the possibility of changing mixing chamber and hence uncertainty in the actual mixing chamber volume we have an excess amount of $\mathrm{He}^{3}$ in the initial helium charge.

In starting up, all the helium is condensed into the dilution refrigerator by opening the valves from the storage volume into both the still and condenser sides. When the pressure is low enough the mechanical pump may be used to evacuate the storage volume, which is then closed of $f$, the remaining gas in the pump body and other dead volumes being allowed to condense to the final equilibrium pressure of a few mm-Hg. Without still power, helium is then circulated. The whole refrigerator then attains a rather stable temperature of half a degree or less. The still power is then turned on to obtain whatever circulation rate is desired (this is usually the most the pump will take). We then wait for equilibrium to be obtained. If the initial mixture is correct, the dilution refrigerator will then cool down steadily to its final low temperature in which the phase boundary lies in the mixing chamber. However, as we mentioned above, the amount of $\mathrm{He}^{3}$ is usually in excess of the correct amount. In the experiments with Refrigerator I, there was enough $\mathrm{He}^{3}$ to fill not only the entire concentrated side but also the entire dilute side of the heat exchangers and on up into the still as well. In this case, $\mathrm{He}^{3}$ was eventually floating on top of superfluid in the still. This initial equilibrium corresponded to a rather uniform temperature of about $1 / 20 \mathrm{~K}$ throughout the entire device. At this point the inlet valve to the condenser was closed, the valve to the storage volume opened, and the pumped gas stored. At first the $\mathrm{He}^{3} / \mathrm{He}^{4}$ ratio was rather large, in excess of $20 / 1$, and the still started to cool while the exchangers and the mixing chamber remained at constant temperature. After some time the still sudden ly started to warm, the $\mathrm{He}^{3} / \mathrm{He}^{4}$ ratio started to drop, and the first exchanger started to cool. Somewhat later the second exchanger started to cool, the first exchanger was thereby warmed, but the temperatures of the remainder of the exchangers and the mixing chamber did not change. As time went on, the "wave" of cold moved down the exchangers and finally reached the mixing chamber. At this time the valve to the storage volume was closed and gas recirculated. The stable low temperature operating condition was then achieved. More gas can then be removed, but so long as the amount removed is not enough to take the phase boundary out of the mixing chamber, the operating conditions in equilibrium are unchanged.

In the experiments with Refrigerator II the heat exchanger dead volumes were so large that the rather arbitrarily determined abount of $\mathrm{He}^{3}$ in the system was not enough to fill the entire dilute side of the exchangers, so the temperature achieved before removing gas was much lower. Once the correct mixture has been obtained, the dilution refrigerator will reach its ultimate stable operating condition starting with the gaseous mixture without special precautions or "prodding" .

The length of time required to establish equilibrium after changes are made in the operating conditions is also important. According to our experience this time is properly estimated by the length of time required to circulate the $\mathrm{He}^{3}$ once through the refrigerator. A high circulation rate and a low dead volume for $\mathrm{He}^{3}$ thus lead to short thermal transients. 


\section{B. Steady-state characteristics}

One type of study which has been made in the present work is a more or less systematic investigation of the effect of the number, size, and flow impedance of the heat exchangers. The results of this study are summarized in Table 8 where we refer to Tables 3, 6, and 7, for specific details on the exchangers and other parts of the apparatus. Temperatures for all parts but the mixing chamber were obtained with precalibrated resistance thermometers. Values of the temperatures derived from these resistors at low temperatures, particularly below $0.1^{\circ} \mathrm{K}$, must be regarded as having only qualitative significance. Relative values for the same resistance thermometers, however, do have significance. Early experiments on Refrigerator I with two heat exchangers, antedating those shown on the first line of Table 8, were performed with $0.25 \mathrm{~mm}$ i.d. interconnecting tubes throughout. The mixing chamber temperature did not go below $65 \mathrm{~m}^{\circ} \mathrm{K}$ with zero power input; the $\mathrm{He}^{3} / \mathrm{He}^{4}$ ratio was not much in excess of 2 or 3 ; and the refrigeration capability was extremely poor. Replacing these tubes on the dilute side by $0.64 \mathrm{~mm}$ i.d. tubes indicated in Table 3 led to the much improved characteristics indicated for two exchangers in Table 8. On adding exchangers, the temperature of any given exchanger did not change very much, and there was a steady decrease in the temperature achieved by the mixing chamber. However, the mixing chamber was not at all as cold as had been expected on the basis of the type of thermodynamical analysis given in equations (75) and (76). We hypothesized that the unexpectedly high value of $T_{1}$ was caused by the heat of separation of the circulated He${ }^{4}$. This was checked by switching over from the circulated mixture to a source of pure $\mathrm{He}^{3}$, the pumped gas being stored. Naturally such an experiment can only be done for a short time, but $T_{1}$ had decreased to about $0.2^{\circ} \mathrm{K}$ before the mixture was again recirculated. Inspection of Table 8 shows that although the mixing chamber temperature $T_{M C}$ did decrease progressively as more exchangers were added, the change from 3 to 4 in Refrigerator I did not produce a large effect. On the basis of these experiments we concluded that the design should be improved to decrease the amount of $\mathrm{He}^{4}$ circulated and to increase the heat exchange capability of the exchangers while decreasing their impedance. The experimental work on the still described in Section IX, A, a was then performed and the changes in the exchangers made as indicated by comparison of Tables 6 and 7. The main impedance was also increased to give a greater margin of safety on the condensation pressure. This was not possible in the pumping circuit for Refrigerator I owing to the limitations of a mercury pump [59] which was used to circulate the $\mathrm{He}^{3}$. The results of these changes are shown in Table 8. There was a uniform improvement in all aspects of the device. The $\mathrm{He}^{3} / \mathrm{He}^{4}$ ratio was improved, this being reflected in a lower value for $T_{1}$. The mixing chamber reached $12 \mathrm{~m}^{\circ} \mathrm{K}$ rather than $21 \mathrm{~m}^{\circ} \mathrm{K}$ as in Refrigerator $\mathrm{I}$. We then added a fifth exchanger, which resulted in a lowering of $T_{M C}$ to $10 \mathrm{~m}^{\circ} \mathrm{K}$. This improvement was sufficiently small as to suggest that the device was once again being limited by some low temperature phenomenon. The results shown in the last line of Table 8 were obtained with tubes on the concentrated side of $0.27 \mathrm{~mm}$ i.d. The $Z$ factor for the tube interconnecting $\mathrm{MC}$ and $\mathrm{fifth}$ exchanger was about $8 \times 10^{8} \mathrm{~cm}^{-3}$. This seemed a bit high according to equation (57), so it was exchanged for a tube of $0.64 \mathrm{~mm}$ i.d. The resulting temperature was rather higher than obtained before. Moreover, another problem occurred in which at a still power of $1 \mathrm{~mW}$ there was excessive heating of the first exchanger, particularly immediately after dumping $\mathrm{He}^{3}$ into the storage volume during the start-up procedure. This problem was correctly diagnosed as caused by vapor formation in the still exchanger. The pressure drop in the $0.27 \mathrm{~mm}$ i.d. tube which had been removed was large enough, due to the high viscosity of $\mathrm{He}^{3}$ at low $T$, to prevent this effect in the earlier version. The problem was corrected by by inserting the vapor suppressing impedance, Section IX, A, e, but no decrease in $T_{M C}$ was obtained. Examination of equation (68) in conjunction with Table 6 leads one to conclude that the $Z$ factor of the dilute side of the last exchanger is too high. Viscous heating of the $\mathrm{He}^{3}$ in this exchanger causes an excessive loss of refrigeration capability. Furthermore, equation (56) and the ensuing discussion suggest that more heat exchange capability in the dilute side of the last exchanger may be necessary if lower mixing chamber temperatures are to be obtained. 


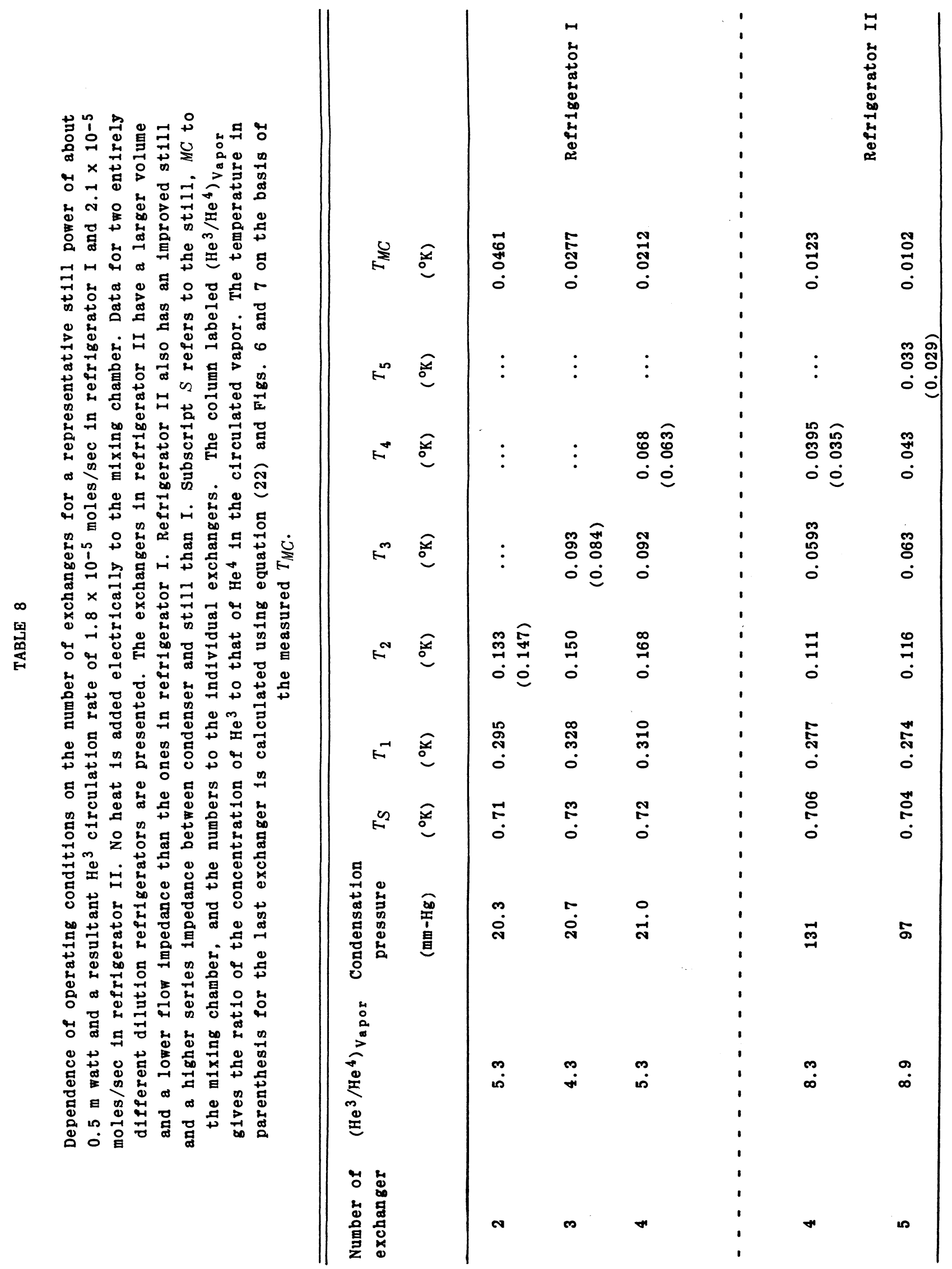


The present temperatures are still substantially above those determined by intrinsic limitations. It seems futile to increase the number of exchangers. Rather the present number of four or five should be adequate, but the qualities of the two lowest ones should be improved.

The temperature of the mixing chamber in the absence of an external heat load depends weakly on the power dissipated in the still. Ideally, $T_{M C}$ should be independent of circulation rate. In practice, as the circulation rate increases, the temperature is bound to rise as a result of the effects of viscosity, imperfect heat exchange, and, ultimately, a decreasing $\mathrm{He}^{3} / \mathrm{He}^{4} \mathrm{ratio}$ At very low circulation rates the $\mathrm{He}^{3} / \mathrm{He}^{4}$ ratio can become so small that heat of separation effects become large and cause the operation of the whole refrigerator to deteriorate. Some operational data in this connection are given in Fig. 19. The variation of $T_{M C}$ with $\dot{Q}_{\mathrm{St} i l 1}$ is

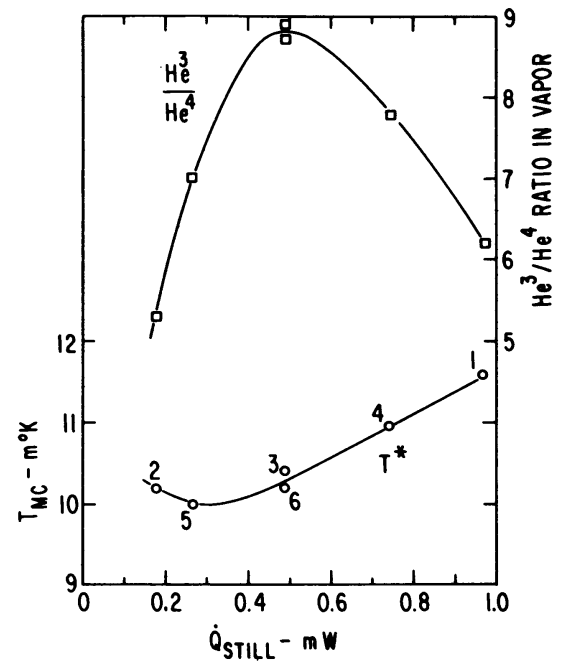

FIGURE 19

Variation in mixing chamber temperature, $T_{M C}^{*}$, and in the ratio of $\mathrm{He}^{3}$ to $\mathrm{He}^{4}$ in the circulated vapor as a function of the st111 power for Refrigerator II and 5 exchangers. The numbers next to the points of the $T^{*}$ curve indicate the order in which the points were obtained. A slow cooling for any given still power was observed over the several day duration of these experiments.

difficult to measure as a result of long-term drift, generally toward lower $T$ as $t$ ime goes on. The dependence of the $\mathrm{He}^{3} / \mathrm{He}^{4}$ ratio on $\dot{Q}_{\mathrm{St} i 11}$ has the same qualitative features suggested in our discussion in Section VI. These data may be analyzed to study the He film flow rate and check semiquantitatively the results of the analysis in Section VI. Results of the analysis are given in Table 9. A reasonably consistent result, considering the accuracy and precision of the data, is obtained. It corresponds to a film flow rate per unit length of approximately $2 \times 10^{-4}$ $\mathrm{cm}^{3} / \mathrm{sec} / \mathrm{cm}$. This is between two and three times greater than the rilm flow rate found for flow over 
clean glass, but is quite comparable to flow rates found for metal surfaces [44].

Not only the limiting temperature but also the external refrigeration canability at higher temperatures are important for the dilution refrigerator. Pesults for both Refrigerators $I$ and II and for various still powers and resulting circulation rates are given in Fig. 20. As we indicate later the results are reasonably consistent with our thermodynaric analysis. The

\section{TABLE 9}

He 4 film flow analysis. Here $r$ is the measured ratio of $\mathrm{He}^{3}$ to $\mathrm{He}^{4}$ in the circulated gas, and $\rho\left(T_{\mathrm{Stil1}}\right)$ is the ratio of $\mathrm{He}^{3}$ to $\mathrm{He}^{4}$ in the vapor above the still calculated for the measured still temperature $T_{S t i l l}$ as the ratio of the $\mathrm{He}^{3}$ vapor pressure to that of pure $\mathrm{He}^{4}$ at temperature $T_{\mathrm{St} \text { ill }}$; see equation $(60)$ and Table 2.

The molar flow rate for $\mathrm{He}^{3}, \dot{n}_{3} / A_{0}$, is obtained from the values calculated in Table 11 and from similar data not presented here. $\dot{Q}_{H C}=0 . \dot{n}_{4_{\mathrm{Fi}} \mathrm{in}_{\mathrm{n}}} / A_{0}$ is the maximum molar $\mathrm{He}^{4}$ flow rate due to film flow calculated from $\dot{n}_{4} \mathrm{Film}=\dot{n}_{3}\left(r^{-1}-\rho^{-1}\right)$. Orifice perimeter is $0.202 \mathrm{~cm}$.

$\begin{array}{llllll}\dot{Q}_{\text {St ill }} & T_{\text {Stil1 }} & r & \rho\left(T_{\mathrm{Stil1}}\right) & \dot{n}_{3} / A_{0} & \dot{n}_{4 \mathrm{Film}} / A_{0}\end{array}$

$(\mathrm{mW}) \quad\left({ }^{\circ} \mathrm{K}\right) \quad\left(10^{-5} \mathrm{moles} / \mathrm{sec}\right) \quad\left(10^{-6} \mathrm{moles} / \mathrm{sec}\right)$

$\begin{array}{lccccc}0.175 & 0.579 & 5.3 & 178 & 0.7 & 1.3 \\ 0.262 & 0.621 & 7.0 & 87 & 1.1 & 1.4 \\ 0.487 & 0.707 & 8.7 & 25.3 & 2.0 & 1.5 \\ 0.743 & 0.764 & 7.8 & 12.8 & 2.7 & 1.4 \\ 0.970 & 0.814 & 6.2 & 7.7 & 3.2 & 1.0\end{array}$

refrigeration capability of the 5-exchanger device in II with a $1 \mathrm{~mW}$ still power is really quite adequate for a variety of low-temperature experiments. Details of the operating characteristics for this configuration are given in Table 10. It is important to note that there is no problem in carrying the mixing chamber to $0.3^{\circ} \mathrm{K}$, where the present refrigerator overlaps the conventional $\mathrm{He}^{3}$ refrigerator. Thus by varying the mixing chamber power we can vary the temperature by a factor of 30. At higher mixing chamber temperatures the $\mathrm{He}^{3} / \mathrm{He}^{4}$ ratio increases substantially. The still temperature drops as a result of the increased concentration of $\mathrm{He}^{3}$ in the dilute solution and the large amount of refrigeration being absorbed at the still.

The data given in Table 10 as well as our other similar data have been analyzed in terms of the thermodynamic discussion in Sections III, IV and VIII. To check the behavior of the mixing chamber, equation (22), it is necessary to know both the temperature of the incoming concentrated $\mathrm{He}^{3}$ and the $\mathrm{He}^{3}$ circulation rate. Neither is known particularly well. The resistance thermometer is not reliable at the lowest temperatures. The $\mathrm{He}^{3}$ circulation rate can be estimated, given the $\mathrm{He}^{3} / \mathrm{He}^{4}$ ratio, by shutting off the cryostat input and observing the rate of 
TABLE 10

Operating characteristics of Refrigerator II with five heat exchangers. The data for $\dot{Q}_{\mathrm{St} \text { il }}=0.490 \mathrm{~mW}$ were obtained with a different set of interconnecting tubes on the concentrated side from the set in operation for the $0.956 \mathrm{~mW}$ data. See the caption to Fig. 20 for these changes. $T_{i}$ is the temperature of the $i$-th exchanger.

\begin{tabular}{|c|c|c|c|c|c|c|c|c|c|c|}
\hline $\begin{array}{c}\dot{Q}_{\mathrm{St} i 11} \\
(\mathrm{~mW})\end{array}$ & $(\mathrm{erg} / \mathrm{sec})$ & $\begin{array}{c}T_{\mathrm{St} i 11} \\
\left({ }^{\circ} \mathrm{K}\right)\end{array}$ & $T_{1}$ & $T_{2}$ & $T_{3}$ & $T_{4}$ & $T_{5}$ & $T_{M C}$ & $\begin{array}{l}\text { Condensation } \\
\text { pressure } \\
(\mathrm{mm}-\mathrm{Hg})\end{array}$ & $\begin{array}{l}\mathrm{He}^{3} / \mathrm{He}^{4} \\
\text { vapor } \\
\text { rat } 10\end{array}$ \\
\hline 0.490 & 0 & 0.710 & 0.274 & 0.116 & 0.063 & 0.044 & 0.035 & 0.0106 & 86 & 8.4 \\
\hline 0.490 & 0.645 & 0.710 & 0.274 & 0.116 & 0.063 & 0.044 & 0.035 & 0.0123 & 85 & 8.8 \\
\hline 0.490 & 5.78 & 0.702 & 0.272 & 0.117 & 0.066 & 0.048 & 0.041 & 0.0218 & 84 & 8.6 \\
\hline 0.490 & 15.75 & 0.695 & 0.272 & 0.121 & 0.072 & 0.055 & 0.052 & 0.0332 & 84 & 9.5 \\
\hline 0.490 & 43.35 & 0.685 & 0.273 & 0.128 & 0.086 & 0.073 & 0.075 & 0.0540 & 83 & 9.6 \\
\hline 0.490 & 84.6 & 0.672 & 0.276 & 0.142 & 0.106 & 0.096 & 0.102 & 0.0757 & 84 & 11.7 \\
\hline 0.490 & 196.9 & 0.635 & 0.286 & 0.174 & 0.152 & 0.148 & 0.157 & 0.1210 & 87 & 14.1 \\
\hline 0.490 & 869. & 0.512 & 0.401 & 0.365 & 0.365 & 0.375 & 0.355 & 0.295 & 100 & 27.1 \\
\hline 0.956 & 0 & 0.828 & 0.330 & 0.141 & 0.073 & 0.047 & 0.038 & 0.0113 & 68 & 6.5 \\
\hline 0.956 & 1.958 & 0.829 & 0.328 & 0.142 & 0.074 & 0.048 & 0.039 & 0.0145 & 67 & 6.5 \\
\hline 0.956 & 10.56 & 0.827 & 0.327 & 0.141 & 0.075 & 0.051 & 0.044 & 0.0239 & 67 & 6.5 \\
\hline 0.956 & 43.1 & 0.809 & 0.318 & 0.145 & 0.084 & 0.066 & 0.063 & 0.0441 & 68 & 7.8 \\
\hline 0.956 & 84.1 & 0.806 & 0.318 & 0.148 & 0.096 & 0.079 & 0.081 & 0.0596 & 71 & 8.6 \\
\hline 0.956 & 199.9 & 0.784 & 0.314 & 0.163 & 0.122 & 0.121 & 0.118 & 0.0891 & 78 & 10.7 \\
\hline 0.956 & 940. & 0.727 & 0.359 & 0.271 & 0.256 & 0.257 & 0.273 & 0.1783 & 100 & 24.6 \\
\hline
\end{tabular}

rise of pressure in a known volume. This method underestimates the equilibrium flow rate, as may be deduced from the transient behavior of the data. Hence we choose to look for consistency in the calculated $\mathrm{He}^{3}$ flow rate. The analysis is presented in Table 11 for the five-exchanger arrangement and for two different still powers. Quite reasonable consistency is obtained for $\dot{n}_{3}$. The magnitudes of the calculated $\mathrm{He}^{3}$ flow rates are entirely reasonable in comparison with the flow rates as measured above. 
As might be expected from our earlier discussion of the shortcomings of the heat exchangers, it is not surprising to find that when their operating temperatures are compared with those expected for ideal conditions the agreement is not particularly good. The figures worked out at the end of Section VIII may be compared with those obtained in one particular run with the fiveexchanger arrangement in which the mixing chamber temperature was about $10 \mathrm{~m}$ oK. The fifth exchanger should then have been at $28 \mathrm{~m}^{\circ} \mathrm{K}$; it was actually at $33 \mathrm{~m}^{\circ} \mathrm{K}$ though this figure is

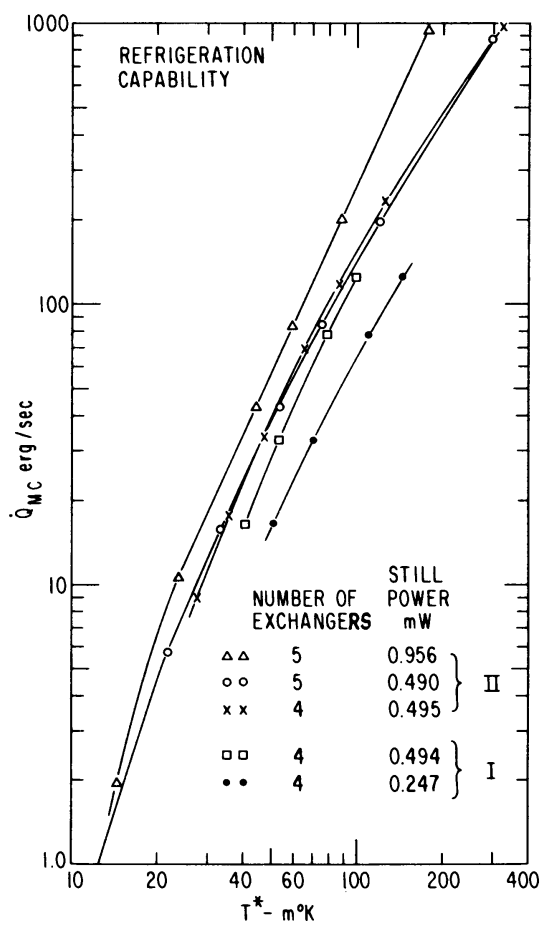

FIGURE 20

\begin{abstract}
Refrigeration capability of the continuous dilution refrigerator for various still powers and for two completely different refrigerators, I and II. The data for 5 exchangers (II) and $0.49 \mathrm{~mW}$ were obtained with different interconnecting tubes on the concentrated side than those for 5 exchangers (II) and $0.956 \mathrm{~mW}$. In the former only $0.27 \mathrm{~mm}$ bore tubing was used. In the latter $0.64 \mathrm{~mm}$ bore tubing was used below exchanger 4 on the concentrated side and a vapor-suppressing impedance of $0.1 \mathrm{~mm}$ bore tubing was interposed between the still and exchanger 1 , also on the concentrated side. Characteristics of refrigerators I and II are listed in Table 3 .
\end{abstract}

extremely unreliable. However, the fourth exchanger should have been at $68 \mathrm{~m}^{\circ} \mathrm{K}$; it was actually at $43 \mathrm{~m}^{\circ} \mathrm{K}$. The third exchanger should then have been at $185 \mathrm{~m}^{\circ} \mathrm{K}$; it was actually at $63 \mathrm{~m}{ }^{\circ} \mathrm{K}$. The reduction in temperature from exchanger to exchanger is not at all as much as should have 
occurred under ideal conditions. This is particularly evident in comparing the third and fourth lines of Table 8, where two different dilution refrigerators with the same number of exchangers are compared. A substantial improvement was made in Refrigerator II, but the lowest exchangers still do not work perfectly and the circulated $\mathrm{He}^{4}$ still leads to a substantial heat load on the first exchanger.

\section{TABLE 11}

Thermodynamic analysis of the operation of the mixing chamber when it is heated electrically. Data are given for a five exchanger arrangement.

$\Delta u_{3_{c}} / k=\left[u_{3_{c}}\left(T_{5}\right)-u_{3_{c}}\left(T_{M C}\right)\right] / k$ is evaluated using the curve on Fig. 7 from experimental values of $T_{5}$ and $T_{M C} . T_{M C} \Delta s\left(T_{M C}\right) / k$ is evaluated using the curve on Fig. 6 from the experimental value of $T_{i M C}$. $\dot{Q}_{\text {effective }} / \dot{n}_{3} k=T_{M C} \Delta s\left(T_{M C}\right) / k-$ $\Delta u_{3_{c}} / k . \quad \dot{n}_{3} / A_{0}$, the calculated He ${ }^{3}$ molar flow rate, is obtained by dividing $\dot{Q}_{i C C} / R$ by $\dot{Q}_{\text {effective/ }} / \dot{n}_{3} k$, following the thermodynamic result given in equation (22). A $A_{0}$ is Avogadro's number, $R$ the gas constant, and $k$ Boltzmann's constant.

\begin{tabular}{lccccccc}
\hline$\dot{Q}_{\mathrm{St} \mathrm{il}}$ & $\dot{Q}_{M C}$ & $T_{5}$ & $T_{M C}$ & $\frac{\Delta_{u_{3}}}{k}$ & $\frac{T_{M C} \Delta s\left(T_{M C}\right)}{k}$ & $\frac{\dot{Q}_{\mathrm{eff}}}{\dot{n}_{3} k}$ & $\frac{\dot{n}_{3}}{A_{0}}$ \\
$(\mathrm{~mW})$ & $(\mathrm{erg} / \mathrm{sec})$ & $\left({ }^{\circ} \mathrm{K}\right)$ & $\left({ }^{\circ} \mathrm{K}\right)$ & $\left({ }^{\circ} \mathrm{K}\right)$ & $\left({ }^{\circ} \mathrm{K}\right)$ & $\left({ }^{\circ} \mathrm{K}\right)$ & $\left(10^{-5} \mathrm{moles} / \mathrm{sec}\right)$ \\
\hline 0.490 & 5.76 & 0.041 & 0.0218 & 0.00164 & 0.00477 & 0.00313 & 2.2 \\
0.490 & 15.75 & 0.052 & 0.0332 & 0.00205 & 0.0110 & 0.0090 & 2.1 \\
0.490 & 43.35 & 0.075 & 0.0540 & 0.0033 & 0.0285 & 0.0252 & 2.1 \\
0.490 & 84.6 & 0.102 & 0.0757 & 0.0053 & 0.0535 & 0.0482 & 2.1 \\
0.956 & 10.56 & 0.0435 & 0.0239 & 0.0017 & 0.00570 & 0.0040 & 3.2 \\
0.956 & 43.1 & 0.0631 & 0.0441 & 0.0025 & 0.0193 & 0.0168 & 3.1 \\
0.956 & 84.1 & 0.0811 & 0.0596 & 0.0036 & 0.0345 & 0.0309 & 3.3 \\
0.956 & 199.9 & 0.1175 & 0.0891 & 0.0065 & 0.0710 & 0.0645 & 3.7 \\
\hline
\end{tabular}

\section{Acknowledgements}

We wish to acknowledge the assistance of Mr. lösta Ehnholm and Mr. David Hamblen in some of our preliminary studies and of Mr. Richard Johnson in other developmental work. We are indebted to Dr. A.C. Mota for checking most of the numerical wors in this paper. We wish to thank Mr.Gene Hirschkoff for a careful reading of the manuscript. It is a pleasure to thank Mrs. Maxine Boyl 
for her secretarial assistance. We are particularly grateful to Mr. Gene A. Porter for his skillful part in the mechanical construction of Refrigerator II.

\section{References}

1. H. LONDON, Proc. Int. Conf. Low Temperature Physics, p. 157. Oxford (1951).

2. L.D. LANDAU and I. POMERANCHUK, Dokl. Akad. Nauk SSSR 59, 669 (1948).

3. I. POMERANCHUK, Zh. Eks. Teor, Fiz, 19, 42 (1949).

4. G.K. WALTERS and W.M. FAIRBANK, Phys. Rev.'103, 262 (1956).

5. H. LONDON, G.R. CLARKE and E. MENDOZA, Phys. Rev. 128, 1992 (1962).

6. T.P. DAS, R. DE BRUYN OUBOTER and K. T. TACONIS, Proc. 9th Int. Conf. Low Temperature Physics, Columbus, Ohio, eds. J.G. DAUNT, D.0. EDWARDS, F.J. MILFORD and M. YAQUB, Part B, p. 1253, Plenum Press, New York (1965).

7. H.E. HALL, P.J. FORD and K. THOMPSON, Cryogenics 6, 80 (1966).

8. B.S. NEGANOV, N. BORISOV and M. LIBURG, Zh. Eksp. Teor. Fiz. 50, 1445 (1966); [Engl1sh translation: Soviet Phys. JETP 23, 959 (1966)].

9. O.E. VILCHES and J.C. MHEATLEY, privately circulated report (1966), unpublished.

10. O.E. VILCHES and J.C. WHEATLEY, Phys. Letters 24A, 440 (1967); 25A, 344 (1967).

11. J.C. WHEATLEY, Am. J. Phys. 36, 181 (1968).

12. A.C. ANDERSON, D.O. EDWARDS, W.R. ROACH, R.E. SARWINSKI and J.C. MHEATLEY, Phys. Rev. Letters 17, 367 (1966).

13. E.C. STONER, Phil. Mag. 25, 889 (1938). See Tables Ia and Ib.

14. J. MCDOUgall and E.C. STONER, Phil. Trans. R. Soc. Lond. A237, 67 (1938).

15. J. BARDEEN, G. BAYM and D. PINES, Phys. Rev. 156, 207 (1967).

16. E.C. KERR, Proc. 5th Int. Conf. Low Temperature Physics and Chemistry, Madison, Wisconsin, ed. J.R. DILLINGER, p. 158. University of Wisconsin Press, Madison, Misconsin (1958).

17. C. BOGHOSIAN and H. MEYER, Phys. Letters 25A, 352 (1967).

18. K. T. TACONIS and R. DE BRUYN OUBOTER, Progr. Low Temp. Phys. IV, ed. C.J. GORTER, p. 38. North-Holland, Amsterdam (1964).

19. T.R. ROBERTS and S.G. SYDORIAK, Phys. Rev. 118, 901 (1960).

20. K.N. ZINOV' EVA and V.P. PESHKOV, Zh. Eksp. Teor. Fiz. 37, 33 (1959). [English trans1.: Soviet Phys. JETP 10, $22(1960)]$. 
21. E.H. GRAF, D.M. LEE and J.D. REPPY, Phys. Rev. Letters 19, 417 (1967).

22. E.M. IFFT, D.0. EDWARDS, R.J. SARWINSKI and M.M. SKERTIC, Phys. Rev. Letters, 19, 831 (1967).

23. D. O. EDWARDS and J.G. DAUNT, Phys. Rev. 124, 640 (1961).

24. D.F. BREWER and J.R.G. KEYSTON, Phys. Let ters 1, 5 (1962).

25. Any of a number of equivalent forms for $\mu_{3}$ are possible. That is, one has

$$
\mu_{3}=\left(\frac{\partial U}{\partial N_{3}}\right)_{S, \Omega, N_{4}}=\left(\frac{\partial F}{\partial N_{3}}\right)_{T, \Omega, N_{4}}=\left(\frac{\partial G}{\partial V_{3}}\right)_{T, P, N_{4}} .
$$

26. M.F. WILSON, D.O. EDWARDS and J.T. TOUGH, Phys. Rev. Let ters 19, 1368 (1967).

27. See Ref. 11, discussion leading to equation (48).

28. C. EBNER, Phys. Rev. 156, 222 (1967).

29. W.R. ABEL, R.T. JOHNSON, J.C. WHEATLEY and W. ZIMMERMANN, Jr., Phys. Rev. Let ters 18, 737 (1967).

30. G. BAYM, Phys. Rev. Letters 17, 952 (1966).

31. J.C. WHEATLEY, Quantum Fluids ed. D.F. BREWER, p. 183. North-Holland, Amsterdam (1966).

32. J.C. WheATleY, Phys. Rev. 165, 304 (1968).

33. B.M. ABRAHAM, Y. ECKSTEIN, J.B. KetTeRSON and J.H. VIGNOS, Phys. Rev. Letters 17, 1254 (1966).

34. G. BAYM and C. EBNER, Phys, Rev. 164, 235 (1967).

35. W. R. ROACH, thesis, University of Illinols, 1966, unpublished.

36. T.P. PTUKHA, Zh. Eksp. Teor. Fiz. 40, 1583 (1961) [English transl.: Soviet Phys. JETP 13, $1112(1961)]$.

37. The definitions of $s_{3_{d}}$ and $s_{4 d}$ are $s_{3_{d}}=\left(\frac{\partial S_{d}}{\partial N_{3}}\right)_{T, N_{4}} \quad ; s_{4}=\left(\frac{\partial S_{d}}{\partial N_{4}}\right)_{T, N_{3}}$ similar definitions hold for other partial quantities, such as $u_{3}, u_{4_{d}} ; \mu_{3_{d}} \mu^{\mu_{d}}$; $\omega_{3}, \omega_{4}$, etc. In the above we have taken $P=0$.

38. W.R. ABEL, A.C. ANDERSON, W.C. BLACK and J.C. WHEATLEY, Phys. Rev. 147, 111 (1966).

39. R. DE BRUYN OUBOTER and K.W. TACONIS, unpublished work.

40. P.L. KaPITZA, J. Phys. Moscow 4, 181 (1941). 
41. A.C. ANDERson, J.I. CONNOLly and J.C. WheAtley, Phys. Rev. 135, A910 (1964).

42. I.M. KHALATNIKOV, Zh. Eksp. Teor. Fiz. 22, 687 (1952).

43. J. GAVORET, Phys. Rev. 137, A721 (1965).

44. K. Mendelssohn and G.K. WHITE, Proc. Phys. Soc. A63, 1328 (1950).

45. E. AMBLER and N. KURTI, Phil. Mag. 43, 260 (1952).

46. W.R. ABEL, A.C. ANDERSON, W.C. BLACK and J.C. WHEATLEY, Phys ics 1, 337 (1965), see Section III $-\mathrm{F}$.

47. Manufactured by Wilbur B. Driver Co., 1875 McCarter Highway, Newark 4, N.J.

48. Copper powder is obtained from A.D. Mackay, Inc., 198 Broadway, New York.

49. G. EHNHOLM and J.C. WHEATLEY, Notes on Sintered Copper Heat Exchangers, unpublished, but available from the present authors on request.

50. Furane Plastics, 4516 Brazil Street, Los Angeles, Calff.

51. W.R. ABEL, A.C. ANDERSON and J.C. WHEATLEY, Rev. Sci. Instrum. 35, 444 (1964).

52. J.C. WheAtley, Ann. Acad. Sci. Fennicae A VI, p. 15, No. 210 (1966).

53. D.S. BETTS, D.W. OSBORNE, B. WELKER and J. WILKS, Phi l. Mag. 8, 977 (1963).

54. F.J. SHORE, V.L. SAILOR, H. MARSHAK and C.A. REYNOLDS, Rev. Sci. Instrum. 31, 970 (1960).

55. R. BERMAN, Proc. Phys. Soc. Lond. A65, 1029 (1952).

56. A.C. ANDERSON, W. REESE and J.C. WHEATLEY, Rev. Sci. Instrum. 34, 1386 (1963).

57. Ersin Multicore, 5 Core, $60 \mathrm{Sn}-40 \mathrm{~Pb}$ solder. Multicore Solders, Ltd. England.

58. Linde Co., Division of Union Carbide, 1/16 in. pellets.

59. This pump is the Edwards 2M4. Although this is an excellent pump it is vastiy inferior, operating all by itself, to the combination indicated in Fig. 18, particularly during the starting-up and shutting-down operations. It also has about half the maximum throughput of the B-2 pump when backed by the modified Welch pump.

\section{Appendix A}

Low temperature approximations for a dilute solution

These are based on the experimental observation [17] that the dependence of the internal energy of a dilute solution on temperature at saturated vapor pressure (and hence approximately zero pressure) and fixed concentration is the same as that of an ideal Fermi-Dirac gas $[13,14]$. Thus along a zero pressure isobar 


$$
U\left(T, n_{3}, n_{4}\right)-U\left(0, n_{3}, n_{4}\right)=n_{3} k \frac{\pi^{2}}{4} \frac{T^{2}}{T_{F}},
$$

where the Fermi temperature $T_{F}$ will be assumed to depend on $n_{3}$ and $n_{4}$ by

$$
\Gamma_{F}=\lambda\left(\frac{n_{3}}{n_{4}}\right)^{2 / 3} \text {. }
$$

$\lambda$ being a constant. Then, since $d S=d U / T$, one has

$$
s\left(T, n_{3}, n_{4}\right)=n_{3} k \frac{\pi^{2}}{2} \frac{T}{T_{F}} .
$$

Inserting (A2) inth (A1) and (A3) one finds the explicit forms

$$
U\left(T, n_{3}, n_{4}\right)-I\left(0, n_{3}, n_{4}\right)=k \frac{\pi^{2}}{4} \frac{T^{2}}{\lambda} n_{3}^{1 / 3} n_{4} 2 / 3
$$

and

$$
S\left(T, n_{3}, n_{4}\right)=k \frac{\pi^{2}}{2} \frac{T}{\lambda} n_{3}^{1 / 3} n_{4}^{2 / 3} .
$$

Letting $\Delta$ operating on a quantity represent the change in the quantity from its value at $T=0$ we then have

$$
\begin{aligned}
\Delta u_{3_{d}} & =\left(\frac{\partial \Delta U}{\partial n_{3}}\right)_{T, n_{4}}=\frac{\pi^{2}}{12} \frac{k T^{2}}{\lambda}\left(\frac{n_{4}}{n_{3}}\right)^{2 / 3}=\frac{\pi^{2}}{12} \frac{k T^{2}}{T_{F}}, \\
\Delta u_{4_{d}} & =\left(\frac{\partial \Delta I}{\partial n_{4}}\right)_{T, n_{3}}=x \frac{\pi^{2}}{5} \frac{k T^{2}}{T_{F}}, \\
s_{3_{d}} & =\left(\frac{\partial S}{\partial n_{3}}\right)_{T, n_{4}}=\frac{\pi^{2}}{6} \frac{k T}{T_{F}}, \\
s_{4_{d}} & =\left(\frac{\partial S}{\partial n_{4}}\right)_{T, n_{3}}=x \frac{\pi^{2}}{3} \frac{k T}{T_{F}}, \\
\Delta \mu_{3} & =\Delta u_{3}-T s_{3}=-\frac{\pi^{2}}{12} \frac{k T^{2}}{T_{F}}, \\
\Delta \mu_{4_{d}} & =\Delta u_{4_{d}}-T s_{4_{d}}=-x \frac{\pi^{2}}{5} \frac{k T^{2}}{T_{F}} .
\end{aligned}
$$


Notes added in proof.

(1) We have recently received a useful report by $R$. Radebaugh on the "Thermodynamic Properties of $\mathrm{He}^{3}-\mathrm{He}^{4}$ Solutions with Applications to the $\mathrm{He}^{3}-\mathrm{He}^{4}$ Dilution Refrigerator, "NBS Technical Note 362, Dec. 1967.

(2) In recent experiments by Abel and Wheatley, the general conclusions of Sec. VII on Intrinsic Factors have been strongly supported. 